Supporting Information

\title{
M[TPP]Cl (M = Fe or Mn) Catalyzed Oxidative Amination of Phenols by primary and secondary Anilines
}

\author{
Vlada Vershinin and Doron Pappo* \\ Department of Chemistry, Ben-Gurion University of the Negev, Beer-Sheva 84105, Israel \\ Email: pappod@bgu.ac.il
}

\section{$\underline{\text { Table of contents }}$}

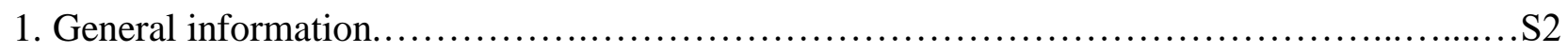

2. Experimental data.......................................................... $3-26$

2.1 Oxidative cross-coupling between phenols and anilines............................ $3-14$

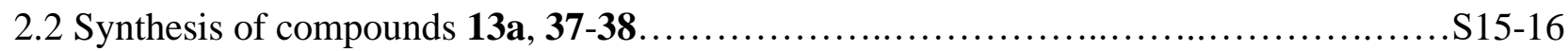

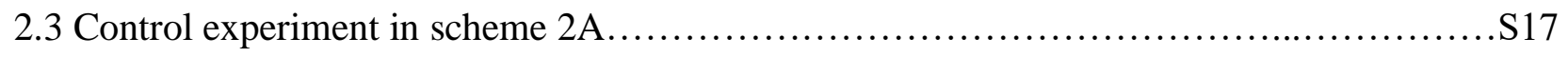

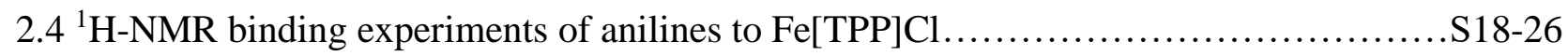

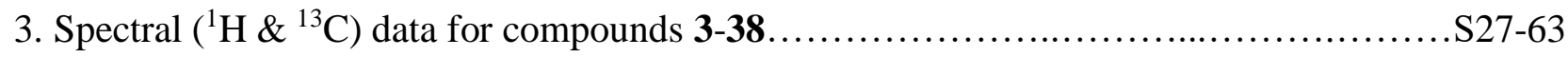




\section{General information:}

All reagents were of reagent grade quality, purchased commercially from Sigma-Aldrich, AlfaAesar, or Fluka, and used without further purification. $\mathrm{FeCl}_{3}$ anhydrous $98 \%$ purchased from Strem Chemicals. Purification by column chromatography was performed on Merck chromatographic silica gel $(40-63 \mu \mathrm{m})$. Thin-layer chromatography (TLC) analyses were performed using Merck silica gel glass plates 60 F254. NMR spectra were recorded on Bruker DPX400 or DMX500 instruments; chemical shifts, given in, are relative to $\mathrm{Me}_{4} \mathrm{Si}$ as the internal standard or to the residual solvent peak. High-resolution mass spectrometry (HRMS) data were obtained using a $\mathrm{Q}$ Exactive Focus mass spectrometer (Thermo Fisher Scientific). HPLC analysis was carried out on Agilent 1260 instrument equipped with a G4212-60008 photodiode array detector and an Agilent reverse phase ZORBAX Eclipse plus C18 $3.5 \mu \mathrm{m}$ column (4.6 X $100 \mathrm{~mm}$ ). 


\section{Experimental data:}

\subsection{Oxidative cross-coupling between phenols and anilines:}

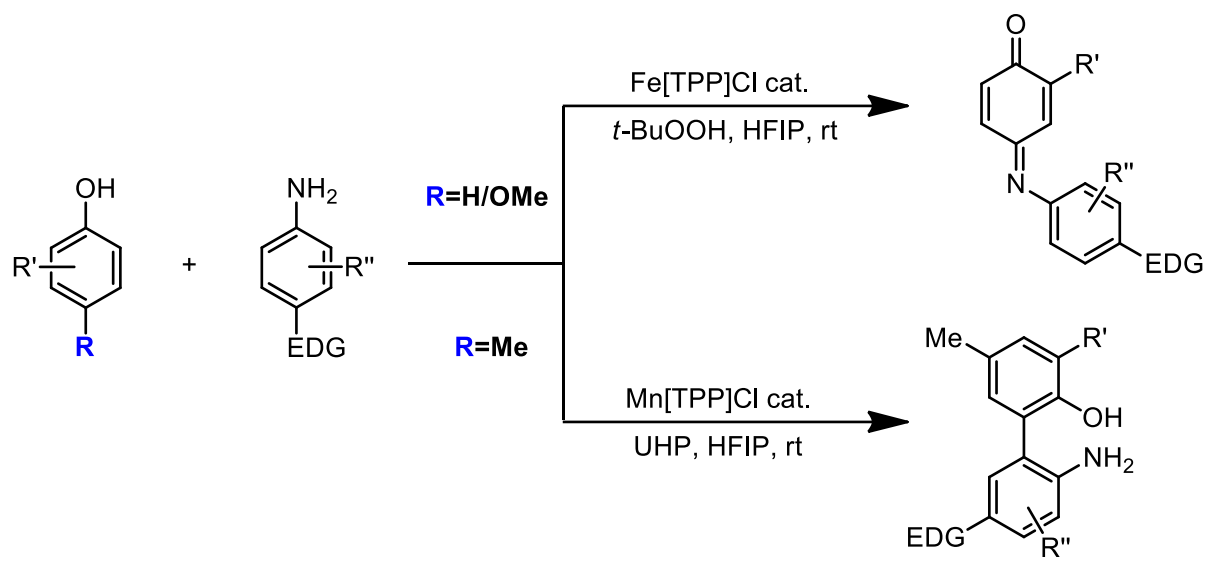

\subsubsection{General procedures:}

Method A: To a stirred solution of phenol (1.5 equiv), aniline (1.0 equiv) and $\mathrm{Fe}[\mathrm{TPP}] \mathrm{Cl}(1 \mathrm{~mol} \%)$ in HFIP $(0.5 \mathrm{M}), t$-BuOOH (2 equiv, $5.5 \mathrm{M}$ solution in decane) was added drop-wise at room temperature and the reaction was stirred for overnight. The volatiles were removed under reduced pressure and the crude was further purified by silica-gel column chromatography (silica gel 40-63 $\mu \mathrm{m})$ affording benzoquinone anil products.

Method B: To a stirred solution of phenol (1.5 equiv), aniline (1.0 equiv) and $\mathrm{Mn}[\mathrm{TPP}] \mathrm{Cl}$ (1 mol $\%)$ in HFIP $(0.5 \mathrm{M})$, UHP (1.5 equiv) was added in one portion at room temperature and the reaction was stirred for overnight. The volatiles were removed under reduced pressure and the crude was further purified by silica-gel column chromatography (silica gel 40-63 $\mu \mathrm{m}$ ) affording $N, O$ biaryl products.

\subsubsection{Typical example for a large-scale oxidative cross-coupling between phenols and anilines:}

To a stirred solution of 2,6-dimethoxyphenol (694 mg, $4.5 \mathrm{mmol}$ ), 3,4-dimethoxyaniline (460 mg, 3 mmol) and $\mathrm{Fe}[\mathrm{TPP}] \mathrm{Cl}(20.4 \mathrm{mg}, 1 \mathrm{~mol} \%)$ in HFIP (6 ml, $0.5 \mathrm{M}), t$-BuOOH $(1.09 \mathrm{ml}, 6 \mathrm{mmol})$ was added drop-wise at room temperature and the reaction was stirred for overnight. The volatiles were removed under reduced pressure and the crude was further purified by column chromatography (hexane/ethyl acetate 40:60) to afford compound $\mathbf{3}$ (641 mg, 70\% yield) as a dark red solid. 


\subsubsection{Characterization data:}<smiles>COC1=CC(=Nc2ccc(OC)cc2)C=C(OC)C1=O</smiles>

Compound 3: 2,6-dimethoxyphenol (57.8 $\mathrm{mg}, 0.375 \mathrm{mmol})$ and 3,4dimethoxyaniline $(38.3 \mathrm{mg}, 0.25 \mathrm{mmol}$ ) were reacted according to method A. The crude residue was purified by column chromatography (hexane/ethyl acetate 40:60) to afford compound $3(59.5 \mathrm{mg}, 78 \%$ yield) as a dark red solid.

Characterization data of compound 3: ${ }^{1} \mathrm{H}$ NMR $\left(400 \mathrm{MHz}, \mathrm{CDCl}_{3}\right) \delta 6.86(\mathrm{~d}, J=8.5 \mathrm{~Hz}, 1 \mathrm{H}), 6.54$ $(\mathrm{d}, J=2.3 \mathrm{~Hz}, 1 \mathrm{H}), 6.39(\mathrm{dd}, J=8.4,2.3 \mathrm{~Hz}, 1 \mathrm{H}), 6.37$ (d, $J=2.1 \mathrm{~Hz}, 1 \mathrm{H}), 6.25(\mathrm{~d}, J=2.1 \mathrm{~Hz}$, $1 \mathrm{H}), 3.88(\mathrm{~s}, 3 \mathrm{H}), 3.85(\mathrm{~s}, 3 \mathrm{H}), 3.83(\mathrm{~s}, 3 \mathrm{H}), 3.66(\mathrm{~s}, 3 \mathrm{H}) ;{ }^{13} \mathrm{C}\left\{{ }^{1} \mathrm{H}\right\} \mathrm{NMR}\left(100 \mathrm{MHz}, \mathrm{CDCl}_{3}\right) \delta$ 176.6, 156.7, 155.5, 154.5, 149.5, 147.1, 143.9, 112.8, 112.1, 111.3, 106.1, 99.0, 56.1, 56.0. HRMS (ESI) m/z: $[\mathrm{M}+\mathrm{H}]^{+}$calcd for $\mathrm{C}_{16} \mathrm{H}_{18} \mathrm{NO}_{5} 304.1179$, found 304.1183 .

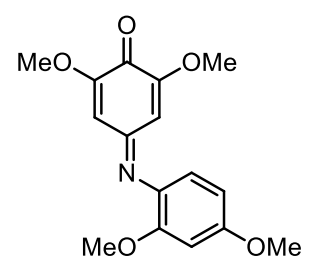

Compound 4: 2,6-dimethoxyphenol (57.8 $\mathrm{mg}, 0.375 \mathrm{mmol})$ and 2,4dimethoxyaniline $(38.3 \mathrm{mg}, 0.25 \mathrm{mmol}$ ) were reacted according to method A. The crude residue was purified by column chromatography (hexane/ethyl acetate 40:60) to afford compound 4 (73.2 $\mathrm{mg}, 96 \%$ yield) as a dark pink solid. Characterization data of compound 4: ${ }^{1} \mathrm{H}$ NMR $\left(400 \mathrm{MHz}, \mathrm{CDCl}_{3}\right) \delta 6.65(\mathrm{~d}, J=8.5 \mathrm{~Hz}, 1 \mathrm{H}), 6.56$ $(\mathrm{d}, J=2.5 \mathrm{~Hz}, 1 \mathrm{H}), 6.52(\mathrm{~d}, J=2.2 \mathrm{~Hz}, 1 \mathrm{H}), 6.49(\mathrm{dd}, J=8.5,2.5 \mathrm{~Hz}, 1 \mathrm{H}), 6.20(\mathrm{~d}, J=2.2 \mathrm{~Hz}$, $1 \mathrm{H}), 3.84(\mathrm{~s}, 6 \mathrm{H}), 3.83(\mathrm{~s}, 3 \mathrm{H}), 3.67(\mathrm{~s}, 3 \mathrm{H}) ;{ }^{13} \mathrm{C}\left\{{ }^{1} \mathrm{H}\right\} \mathrm{NMR}\left(100 \mathrm{MHz}, \mathrm{CDCl}_{3}\right) \delta 176.8,159.3$, $157.0,155.2,154.3,152.5,133.1,122.3,112.5,104.1,99.6,99.5,56.2,56.0,55.9$, 55.6. HRMS (ESI) m/z: $[\mathrm{M}+\mathrm{H}]^{+}$calcd for $\mathrm{C}_{16} \mathrm{H}_{18} \mathrm{NO}_{5} 304.1179$, found 304.1181 .

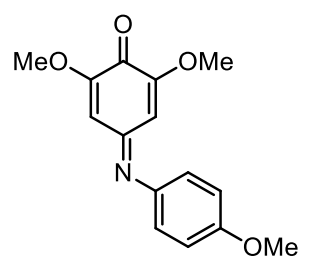

Compound 5: 2,6-dimethoxyphenol (57.8 $\mathrm{mg}, 0.375 \mathrm{mmol})$ and 4methoxyaniline $(30.8 \mathrm{mg}, 0.25 \mathrm{mmol})$ were reacted according to method A. The crude residue was purified by column chromatography (hexane/ethyl Characterization data of compound 5: ${ }^{1} \mathrm{H} \mathrm{NMR}\left(400 \mathrm{MHz}, \mathrm{CDCl}_{3}\right) \delta 6.98-6.91(\mathrm{~m}, 2 \mathrm{H}), 6.90-$ $6.83(\mathrm{~m}, 2 \mathrm{H}), 6.41(\mathrm{~d}, J=2.2 \mathrm{~Hz}, 1 \mathrm{H}), 6.23(\mathrm{~d}, J=2.2 \mathrm{~Hz}, 1 \mathrm{H}), 3.85$ (s, 3H), 3.83 (s, 3H), 3.67 (s, $3 \mathrm{H}) ;{ }^{13} \mathrm{C}\left\{{ }^{1} \mathrm{H}\right\} \mathrm{NMR}\left(100 \mathrm{MHz}, \mathrm{CDCl}_{3}\right) \delta 176.7,157.8,156.6,155.6,154.5,143.6,122.9,114.5$, 112.4, 99.0, 56.2, 56.0, 55.6. HRMS (ESI) m/z: $[\mathrm{M}+\mathrm{H}]^{+}$calcd for $\mathrm{C}_{15} \mathrm{H}_{16} \mathrm{NO}_{4}$ 274.1074, found 274.1072 . 


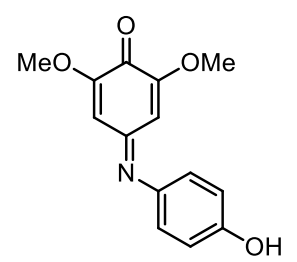

Compound 6: 2,6-dimethoxyphenol (57.8 mg, $0.375 \mathrm{mmol})$ and 4-aminophenol (27.3 $\mathrm{mg}, 0.25 \mathrm{mmol}$ ) were reacted according to method A. The crude residue was purified by column chromatography (hexane/ethyl acetate 50:50) to afford compound 6 (31.2 $\mathrm{mg}, 48 \%$ yield) as a red solid.

Characterization data of compound 6: ${ }^{1} \mathrm{H}$ NMR (400 MHz, DMSO-d6) $\delta 9.60(\mathrm{~s}, 1 \mathrm{H}), 6.83(\mathrm{~s}, 4 \mathrm{H})$, $6.47(\mathrm{~d}, J=2.1 \mathrm{~Hz}, 1 \mathrm{H}), 6.26(\mathrm{~d}, J=2.0 \mathrm{~Hz}, 1 \mathrm{H}), 3.78(\mathrm{~s}, 3 \mathrm{H}), 3.62(\mathrm{~s}, 3 \mathrm{H}) ;{ }^{13} \mathrm{C}\left\{{ }^{1} \mathrm{H}\right\} \mathrm{NMR}(100$ MHz, DMSO-d $d_{6} \delta 175.6,155.7,155.2,154.9,153.9,142.0,123.4,115.8,112.2,98.7,55.9,55.6$. HRMS (ESI) m/z: [M+H] $]^{+}$calcd for $\mathrm{C}_{14} \mathrm{H}_{14} \mathrm{NO}_{4} 260.0917$, found 260.0919 .

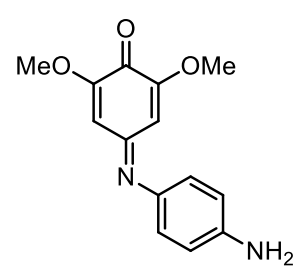

Compound 7: 2,6-dimethoxyphenol (57.8 $\mathrm{mg}, 0.375 \mathrm{mmol})$ and $p$ phenylenediamine $(27 \mathrm{mg}, 0.25 \mathrm{mmol})$ were reacted according to method A. The crude residue was purified by column chromatography (hexane/ethyl acetate 40:60) to afford compound 7 (57.1 mg, $88 \%$ yield) as a dark purple solid.

Characterization data of compound 7: ${ }^{1} \mathrm{H}$ NMR $\left(400 \mathrm{MHz}, \mathrm{CDCl}_{3}\right) \delta 6.85-6.76(\mathrm{~m}, 2 \mathrm{H}), 6.75-$ $6.66(\mathrm{~m}, 2 \mathrm{H}), 6.40(\mathrm{~d}, J=2.2 \mathrm{~Hz}, 1 \mathrm{H}), 6.32(\mathrm{~d}, J=2.2 \mathrm{~Hz}, 1 \mathrm{H}), 3.82(\mathrm{~s}, 3 \mathrm{H}), 3.67(\mathrm{~s}, 3 \mathrm{H}) ;{ }^{13} \mathrm{C}\left\{{ }^{1} \mathrm{H}\right\}$ $\underline{\mathrm{NMR}}\left(100 \mathrm{MHz}, \mathrm{CDCl}_{3}\right) \delta 176.6,155.4,155.3,154.2,145.2,141.8,123.8,115.5,112.5,99.3,56.1$, 55.9. HRMS (ESI) m/z: $[\mathrm{M}+\mathrm{H}]^{+}$calcd for $\mathrm{C}_{14} \mathrm{H}_{15} \mathrm{~N}_{2} \mathrm{O}_{3} 259.1077$, found 259.1081 .

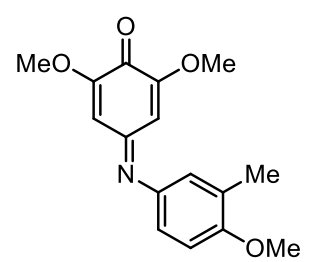

Compound 8: 2,6-dimethoxyphenol (57.8 mg, $0.375 \mathrm{mmol})$ and 4-methoxy-3methylaniline ( $34.3 \mathrm{mg}, 0.25 \mathrm{mmol}$ ) were reacted according to method A. The crude residue was purified by column chromatography (hexane/ethyl acetate $60: 40)$ to afford compound $\mathbf{8}(58.8 \mathrm{mg}, 82 \%$ yield) as a dark red solid.

Characterization data of compound 8: ${ }^{1} \mathrm{H}$ NMR $\left(400 \mathrm{MHz}, \mathrm{CDCl}_{3}\right) \delta 6.83(\mathrm{~d}, J=8.5 \mathrm{~Hz}, 1 \mathrm{H}), 6.77$ $(\mathrm{dd}, J=2.5,0.7 \mathrm{~Hz}, 1 \mathrm{H}), 6.70(\mathrm{dd}, J=8.5,2.7 \mathrm{~Hz}, 1 \mathrm{H}), 6.40(\mathrm{~d}, J=2.2 \mathrm{~Hz}, 1 \mathrm{H}), 6.26(\mathrm{~d}, J=2.2$ $\mathrm{Hz}, 1 \mathrm{H}), 3.84$ (s, 3H), $3.84(\mathrm{~s}, 3 \mathrm{H}), 3.67(\mathrm{~s}, 3 \mathrm{H}), 2.22(\mathrm{~s}, 3 \mathrm{H}) ;{ }^{13} \mathrm{C}\left\{{ }^{1} \mathrm{H}\right\} \mathrm{NMR}\left(100 \mathrm{MHz}, \mathrm{CDCl}_{3}\right) \delta$ 176.7, 156.3, 156.1, 155.4, 154.4, 143.2, 127.6, 124.5, 119.7, 112.4, 110.2, 99.1, 56.1, 56.0, 55.6, 16.4. HRMS (ESI) m/z: $[\mathrm{M}+\mathrm{H}]^{+}$calcd for $\mathrm{C}_{16} \mathrm{H}_{18} \mathrm{NO}_{4} 288.1230$, found 288.1230.

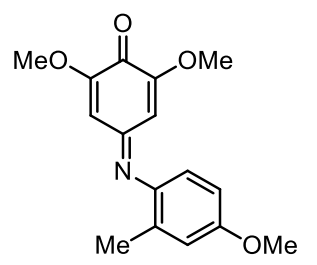

Compound 9: 2,6-dimethoxyphenol (57.8 mg, $0.375 \mathrm{mmol})$ and 4-methoxy-2methylaniline $(32 \mu \mathrm{L}, 0.25 \mathrm{mmol})$ were reacted according to method $\mathrm{A}$. The crude residue was purified by column chromatography (hexane/ethyl acetate 60:40) to afford compound 9 (66.7 $\mathrm{mg}, 93 \%$ yield) as a dark red solid.

Characterization data of compound 9: ${ }^{1} \mathrm{H}$ NMR $\left(400 \mathrm{MHz}, \mathrm{CDCl}_{3}\right) \delta 6.82(\mathrm{~d}, J=2.6 \mathrm{~Hz}, 1 \mathrm{H}), 6.72$ $(\mathrm{dd}, J=8.5,2.6 \mathrm{~Hz}, 1 \mathrm{H}), 6.56(\mathrm{~d}, J=8.5 \mathrm{~Hz}, 1 \mathrm{H}), 6.43(\mathrm{~d}, J=2.2 \mathrm{~Hz}, 1 \mathrm{H}), 6.10(\mathrm{~d}, J=2.2 \mathrm{~Hz}$, 
1H), $3.86(\mathrm{~s}, 3 \mathrm{H}), 3.81(\mathrm{~s}, 3 \mathrm{H}), 3.64(\mathrm{~s}, 3 \mathrm{H}), 2.17(\mathrm{~s}, 3 \mathrm{H}) ;{ }^{13} \mathrm{C}\left\{{ }^{1} \mathrm{H}\right\} \mathrm{NMR}\left(100 \mathrm{MHz}, \mathrm{CDCl}_{3}\right) \delta$ 176.8, 157.7, 156.4, 155.4, 154.4, 142.7, 131.8, 120.8, 116.3, 112.2, 111.2, 99.0, 56.2, 56.0, 55.5, 18.5. HRMS (ESI) m/z: [M+H] $]^{+}$calcd for $\mathrm{C}_{16} \mathrm{H}_{18} \mathrm{NO}_{4} 288.1230$, found 288.1229.

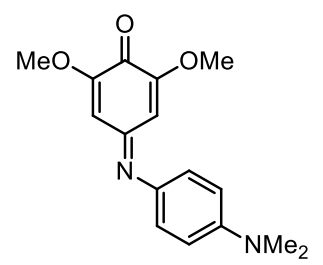

Compound 10: 2,6-dimethoxyphenol (57.8 $\mathrm{mg}, 0.375 \mathrm{mmol})$ and $N, N$ dimethyl-p-phenylenediamine $(34 \mathrm{mg}, 0.25 \mathrm{mmol}$ ) were reacted according to method A. The crude residue was purified by column chromatography (hexane/ethyl acetate 50:50) to afford compound $\mathbf{1 0}$ (49.7 $\mathrm{mg}, 69 \%$ yield) as a dark purple solid.

Characterization data of compound 10: ${ }^{1} \mathrm{H}$ NMR $\left(400 \mathrm{MHz}, \mathrm{CDCl}_{3}\right) \delta 6.96-6.91(\mathrm{~m}, 2 \mathrm{H}), 6.76-$ $6.71(\mathrm{~m}, 2 \mathrm{H}), 6.42(\mathrm{~d}, J=2.2 \mathrm{~Hz}, 1 \mathrm{H}), 6.41(\mathrm{~d}, J=2.2 \mathrm{~Hz}, 1 \mathrm{H}), 3.82$ (s, 3H), 3.69 (s, 3H), 3.00 (s, $6 \mathrm{H}) ;{ }^{13} \mathrm{C}\left\{{ }^{1} \mathrm{H}\right\} \mathrm{NMR}\left(100 \mathrm{MHz}, \mathrm{CDCl}_{3}\right) \delta 176.4,155.3,154.2,154.1,149.1,140.0,124.5,112.8$, 112.5, 99.5, 56.0, 55.9, 40.5. HRMS (ESI) $\mathrm{m} / \mathrm{z}$ : $[\mathrm{M}+\mathrm{H}]^{+}$calcd for $\mathrm{C}_{16} \mathrm{H}_{19} \mathrm{~N}_{2} \mathrm{O}_{3}$ 287.1390, found 287.1388.

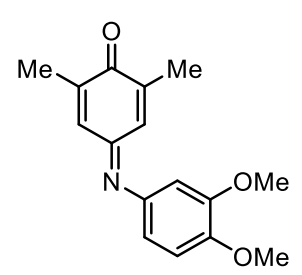

Compound 11: 2,6-dimethylphenol (45.8 $\mathrm{mg}, 0.375 \mathrm{mmol})$ and 3,4dimethoxyaniline ( $38.3 \mathrm{mg}, 0.25 \mathrm{mmol}$ ) were reacted according to method A (in this reaction 3 equiv of $t$ - $\mathrm{BuOOH}$ were added). The crude residue was purified by column chromatography (hexane/ethyl acetate 85:15) to afford compound 11 (28.6 mg, 42\% yield) as a dark red solid.

Characterization data of compound 11: ${ }^{1} \mathrm{H} \mathrm{NMR}\left(400 \mathrm{MHz}, \mathrm{CDCl}_{3}\right) \delta 7.04(\mathrm{dq}, J=2.9,1.4 \mathrm{~Hz}$, $1 \mathrm{H}), 6.94(\mathrm{dq}, J=3.0,1.5 \mathrm{~Hz}, 1 \mathrm{H}), 6.88(\mathrm{~d}, J=8.4 \mathrm{~Hz}, 1 \mathrm{H}), 6.57(\mathrm{~d}, J=2.3 \mathrm{~Hz}, 1 \mathrm{H}), 6.38(\mathrm{dd}, J=$ 8.4, $2.4 \mathrm{~Hz}, 1 \mathrm{H}), 3.91(\mathrm{~s}, 3 \mathrm{H}), 3.87(\mathrm{~s}, 3 \mathrm{H}), 2.08(\mathrm{~d}, J=1.5 \mathrm{~Hz}, 3 \mathrm{H}), 1.99$ (d, $J=1.5 \mathrm{~Hz}, 3 \mathrm{H})$; ${ }^{13} \mathrm{C}\left\{{ }^{1} \mathrm{H}\right\} \mathrm{NMR}\left(100 \mathrm{MHz}, \mathrm{CDCl}_{3}\right) \delta 188.5,157.6,149.5,147.6,143.6,141.7,140.7,138.2,124.9$, 113.1, 111.2, 106.4, 56.2, 56.1, 16.7, 16.2. HRMS (ESI) m/z: $[\mathrm{M}+\mathrm{H}]^{+}$calcd for $\mathrm{C}_{16} \mathrm{H}_{18} \mathrm{NO}_{3}$ 272.1281 , found 272.1277 .

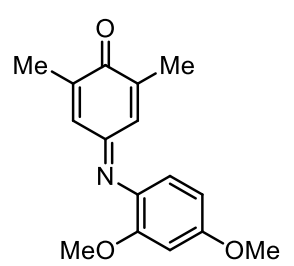

Compound 12: 2,6-dimethylphenol (45.8 mg, $0.375 \mathrm{mmol})$ and 2,4dimethoxyaniline $(38.3 \mathrm{mg}, 0.25 \mathrm{mmol}$ ) were reacted according to method A. The crude residue was purified by column chromatography (hexane/ethyl Characterization data of compound 12: ${ }^{1} \mathrm{H} \mathrm{NMR}\left(400 \mathrm{MHz}, \mathrm{CDCl}_{3}\right) \delta 7.14(\mathrm{dq}, J=2.9,1.4 \mathrm{~Hz}$, $1 \mathrm{H}), 6.88(\mathrm{dq}, J=2.9,1.5 \mathrm{~Hz}, 1 \mathrm{H}), 6.62(\mathrm{~d}, J=8.5 \mathrm{~Hz}, 1 \mathrm{H}), 6.55(\mathrm{~d}, J=2.5 \mathrm{~Hz}, 1 \mathrm{H}), 6.49(\mathrm{dd}, J=$ 8.5, $2.5 \mathrm{~Hz}, 1 \mathrm{H}), 3.83$ (s, 3H), $3.83(\mathrm{~s}, 3 \mathrm{H}), 2.07$ (d, $J=1.5 \mathrm{~Hz}, 3 \mathrm{H}), 1.98$ (d, $J=1.5 \mathrm{~Hz}, 3 \mathrm{H})$; ${ }^{13} \mathrm{C}\left\{{ }^{1} \mathrm{H}\right\} \mathrm{NMR}\left(100 \mathrm{MHz}, \mathrm{CDCl}_{3}\right) \delta 188.6,159.7,157.9,152.6,141.0,140.2,138.4,132.9,125.3$, 
122.6, 104.1, 99.6, 56.0, 55.7, 16.7, 16.1. HRMS (ESI) m/z: $[\mathrm{M}+\mathrm{H}]^{+}$calcd for $\mathrm{C}_{16} \mathrm{H}_{18} \mathrm{NO}_{3}$ 272.1281 , found 272.1279 .

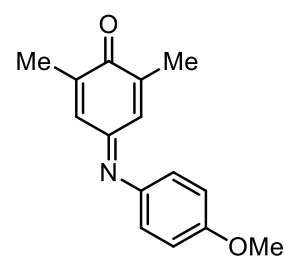

Compound 13: 2,6-dimethylphenol (45.8 $\mathrm{mg}, \quad 0.375 \mathrm{mmol})$ and 4methoxyaniline $(30.8 \mathrm{mg}, 0.25 \mathrm{mmol}$ ) were reacted according to method A. The crude residue was purified by column chromatography (hexane/ethyl acetate 95:5) to afford compound $\mathbf{1 3}$ (33.1 $\mathrm{mg}, 55 \%$ yield) as a dark red syrup.

Characterization data of compound 13: ${ }^{1} \mathrm{H}$ NMR $\left(400 \mathrm{MHz}, \mathrm{CDCl}_{3}\right) \delta 7.04(\mathrm{dq}, J=2.9,1.4 \mathrm{~Hz}$, $1 \mathrm{H}), 6.95-6.91(\mathrm{~m}, 3 \mathrm{H}), 6.88-6.84(\mathrm{~m}, 2 \mathrm{H}), 3.83(\mathrm{~s}, 3 \mathrm{H}), 2.07(\mathrm{~d}, J=1.5 \mathrm{~Hz}, 3 \mathrm{H}), 1.98(\mathrm{~d}, J=$ $1.5 \mathrm{~Hz}, 3 \mathrm{H}) ;{ }^{13} \mathrm{C}\left\{{ }^{1} \mathrm{H}\right\} \mathrm{NMR}\left(100 \mathrm{MHz}, \mathrm{CDCl}_{3}\right) \delta 188.4,158.2,157.3,143.2,141.6,140.5,138.4$, 124.8, 123.2, 114.4, 55.6, 16.7, 16.1. HRMS (ESI) m/z: $[\mathrm{M}+\mathrm{H}]^{+}$calcd for $\mathrm{C}_{15} \mathrm{H}_{16} \mathrm{NO}_{2} 242.1176$, found 242.1174 .<smiles></smiles>

Compound 14: 2,6-dimethylphenol (45.8 mg, $0.375 \mathrm{mmol})$ and 4-aminophenol (27.3 $\mathrm{mg}, 0.25 \mathrm{mmol}$ ) were reacted according to method A. The crude residue was purified by column chromatography (hexane/ethyl acetate 80:20) to afford compound 14 (25.3 mg, 45\% yield) as a red solid.

Characterization data of compound 14: ${ }^{1} \mathrm{H}$ NMR $\left(400 \mathrm{MHz}\right.$, Acetone- $\left.d_{6}\right) \delta 8.67$ (bs, 1H), 7.05 (s, $2 \mathrm{H}), 6.91(\mathrm{~d}, J=8.6 \mathrm{~Hz}, 2 \mathrm{H}), 6.84(\mathrm{~d}, J=8.6 \mathrm{~Hz}, 2 \mathrm{H}), 2.03(\mathrm{~s}, 3 \mathrm{H}), 1.95(\mathrm{~s}, 3 \mathrm{H}) ;{ }^{13} \mathrm{C}\left\{{ }^{1} \mathrm{H}\right\} \mathrm{NMR}$ (100 MHz, Acetone- $\left.d_{6}\right) \delta 188.4,157.3,157.1,143.3,141.8,140.6,139.1,125.4,124.3,116.5,16.4$, 15.9. HRMS (ESI) m/z: $[\mathrm{M}+\mathrm{H}]^{+}$calcd for $\mathrm{C}_{14} \mathrm{H}_{14} \mathrm{NO}_{2} 228.1019$, found 228.1019 .

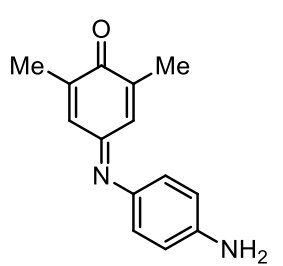

Compound 15: 2,6-dimethylphenol $(45.8 \mathrm{mg}, \quad 0.375 \mathrm{mmol})$ and $p$ phenylenediamine $(27 \mathrm{mg}, 0.25 \mathrm{mmol})$ were reacted according to method A. The crude residue was purified by column chromatography (hexane/ethyl solid.

Characterization data of compound 15: ${ }^{1} \mathrm{H} \mathrm{NMR}\left(400 \mathrm{MHz}, \mathrm{CDCl}_{3}\right) \delta 7.05(\mathrm{dq}, J=2.7,1.3 \mathrm{~Hz}$, 1H), $7.01(\mathrm{dq}, J=2.8,1.4 \mathrm{~Hz}, 1 \mathrm{H}), 6.85-6.78(\mathrm{~m}, 2 \mathrm{H}), 6.75-6.69(\mathrm{~m}, 2 \mathrm{H}), 3.88(\mathrm{bs}, 2 \mathrm{H}), 2.08(\mathrm{~d}$, $J=1.4 \mathrm{~Hz}, 3 \mathrm{H}), 2.00(\mathrm{~d}, J=1.5 \mathrm{~Hz}, 3 \mathrm{H}) ;{ }^{13} \mathrm{C}\left\{{ }^{1} \mathrm{H}\right\} \mathrm{NMR}\left(100 \mathrm{MHz}, \mathrm{CDCl}_{3}\right) \delta 188.6,156.2,145.7$, 141.5, 140.9, 139.9, 138.6, 125.1, 124.4, 115.3, 16.8, 16.2. HRMS (ESI) m/z: $[\mathrm{M}+\mathrm{H}]^{+}$calcd for $\mathrm{C}_{14} \mathrm{H}_{15} \mathrm{~N}_{2} \mathrm{O} 227.1179$, found 227.1179. 


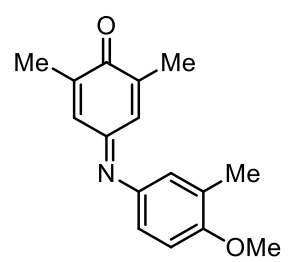

Compound 16: 2,6-dimethylphenol (45.8 mg, $0.375 \mathrm{mmol})$ and 4-methoxy-3methylaniline $(34.3 \mathrm{mg}, 0.25 \mathrm{mmol})$ were reacted according to method A. The crude residue was purified by column chromatography (hexane/ethyl acetate 90:10) to afford compound $\mathbf{1 6}(39.8 \mathrm{mg}, 62 \%$ yield) as a dark red solid.

Characterization data of compound 16: ${ }^{1} \mathrm{H}$ NMR $\left(400 \mathrm{MHz}, \mathrm{CDCl}_{3}\right) \delta 7.05(\mathrm{dq}, J=2.8,1.4 \mathrm{~Hz}$, $1 \mathrm{H}), 6.94(\mathrm{dq}, J=2.9,1.5 \mathrm{~Hz}, 1 \mathrm{H}), 6.84(\mathrm{~d}, J=8.5 \mathrm{~Hz}, 1 \mathrm{H}), 6.76(\mathrm{dd}, J=2.5,0.7 \mathrm{~Hz}, 1 \mathrm{H}), 6.70$ (dd, $J=8.5,2.6 \mathrm{~Hz}, 1 \mathrm{H}), 3.86(\mathrm{~s}, 3 \mathrm{H}), 2.24$ (s, 3H), 2.08 (d, J = 1.5 Hz, 3H), 1.99 (d, J = $1.5 \mathrm{~Hz}$, $3 \mathrm{H}) ;{ }^{13} \mathrm{C}\left\{{ }^{1} \mathrm{H}\right\} \mathrm{NMR}\left(100 \mathrm{MHz}, \mathrm{CDCl}_{3}\right) \delta 188.5,157.1,156.5,143.0,141.4,140.3,138.5,127.5$, 124.9, 124.7, 120.1, 110.2, 55.7, 16.7, 16.4, 16.2. HRMS (ESI) m/z: $[\mathrm{M}+\mathrm{H}]^{+}$calcd for $\mathrm{C}_{16} \mathrm{H}_{18} \mathrm{NO}_{2}$ 256.1332 , found 256.1332 .

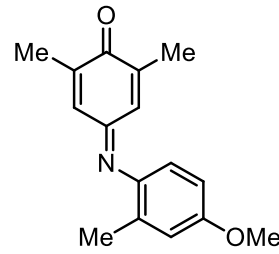

Compound 17: 2,6-dimethylphenol (45.8 mg, $0.375 \mathrm{mmol})$ and 4-methoxy-2methylaniline (32 $\mu \mathrm{L}, 0.25 \mathrm{mmol}$ ) were reacted according to method $\mathrm{A}$. The crude residue was purified by column chromatography (hexane/ethyl acetate 95:5) to afford compound 17 (46.3 $\mathrm{mg}, 73 \%$ yield) as a dark red solid.

Characterization data of compound 17: ${ }^{1} \mathrm{H}$ NMR $\left(400 \mathrm{MHz}, \mathrm{CDCl}_{3}\right) \delta 7.09(\mathrm{dq}, J=2.9,1.4 \mathrm{~Hz}$, $1 \mathrm{H}), 6.86-6.78(\mathrm{~m}, 2 \mathrm{H}), 6.73(\mathrm{dd}, J=8.5,2.6 \mathrm{~Hz}, 1 \mathrm{H}), 6.53(\mathrm{~d}, J=8.5 \mathrm{~Hz}, 1 \mathrm{H}), 3.81(\mathrm{~s}, 3 \mathrm{H}), 2.18$ $(\mathrm{s}, 3 \mathrm{H}), 2.09(\mathrm{~d}, \mathrm{~J}=1.5 \mathrm{~Hz}, 3 \mathrm{H}), 1.97(\mathrm{~d}, \mathrm{~J}=1.5 \mathrm{~Hz}, 3 \mathrm{H}) ;{ }^{13} \mathrm{C}\left\{{ }^{1} \mathrm{H}\right\} \mathrm{NMR}\left(100 \mathrm{MHz}, \mathrm{CDCl}_{3}\right) \delta$ 188.5, 158.1, 157.2, 142.4, 141.5, 140.4, 138.3, 132.3, 124.9, 121.0, 116.3, 111.1, 55.5, 18.5, 16.6, 16.1. HRMS (ESI) m/z: [M+H] $]^{+}$calcd for $\mathrm{C}_{16} \mathrm{H}_{18} \mathrm{NO}_{2} 256.1332$, found 256.1332 .

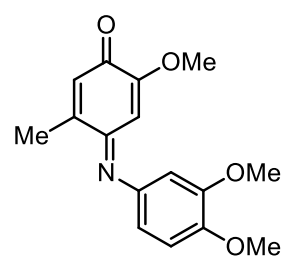

Compound 18: 2-methoxy-5-methylphenol (51.8 mg, $0.375 \mathrm{mmol})$ and 3,4dimethoxyaniline $(38.3 \mathrm{mg}, 0.25 \mathrm{mmol}$ ) were reacted according to method A. The crude residue was purified by column chromatography (hexane/ethyl acetate 65:35) to afford compound $\mathbf{1 8}(50.8 \mathrm{mg}, 71 \%$ yield) as a dark red solid.

Characterization data of compound 18: ${ }^{1} \mathrm{H} \mathrm{NMR}\left(400 \mathrm{MHz}, \mathrm{CDCl}_{3}\right) \delta 6.89(\mathrm{~d}, J=8.4 \mathrm{~Hz}, 1 \mathrm{H}), 6.58$ - $6.52(\mathrm{~m}, 2 \mathrm{H}), 6.40(\mathrm{dd}, J=8.4,2.3 \mathrm{~Hz}, 1 \mathrm{H}), 6.23(\mathrm{~s}, 1 \mathrm{H}), 3.91(\mathrm{~s}, 3 \mathrm{H}), 3.89$ (s, 3H), $3.64(\mathrm{~s}, 3 \mathrm{H})$, $2.29(\mathrm{~d}, J=1.4 \mathrm{~Hz}, 3 \mathrm{H}) ;{ }^{13} \mathrm{C}\left\{{ }^{1} \mathrm{H}\right\} \mathrm{NMR}\left(100 \mathrm{MHz}, \mathrm{CDCl}_{3}\right) \delta 182.2,158.2,155.6,150.2,149.5$, 147.0, 143.9, 129.8, 111.9, 111.3, 105.5, 100.3, 56.2, 56.0, 55.6, 17.9. HRMS (ESI) m/z: [M+H] calcd for $\mathrm{C}_{16} \mathrm{H}_{18} \mathrm{NO}_{4} 288.1230$, found 288.1234. 


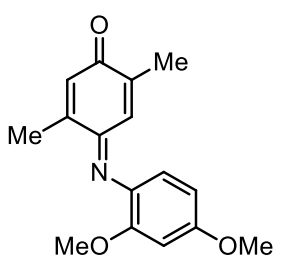

Compound 19: 2,5-dimethylphenol (45.8 $\mathrm{mg}, \quad 0.375 \mathrm{mmol})$ and 2,4dimethoxyaniline $(38.3 \mathrm{mg}, 0.25 \mathrm{mmol})$ were reacted according to method A. The crude residue was purified by column chromatography (hexane/ethyl acetate 90:10) to afford compound $\mathbf{1 9}(17.2 \mathrm{mg}, 25 \%$ yield) as a dark pink syrup.

Characterization data of compound 19: ${ }^{1} \mathrm{H} \mathrm{NMR}\left(400 \mathrm{MHz}, \mathrm{CDCl}_{3}\right) \delta 6.80$ (q, $\left.J=1.5 \mathrm{~Hz}, 1 \mathrm{H}\right), 6.63$ $(\mathrm{d}, J=8.5 \mathrm{~Hz}, 1 \mathrm{H}), 6.56(\mathrm{~d}, J=2.5 \mathrm{~Hz}, 1 \mathrm{H}), 6.54-6.50(\mathrm{~m}, 2 \mathrm{H}), 3.84(\mathrm{~s}, 3 \mathrm{H}), 3.80(\mathrm{~s}, 3 \mathrm{H}), 2.30$ $(\mathrm{d}, J=1.4 \mathrm{~Hz}, 3 \mathrm{H}), 1.93(\mathrm{~d}, J=1.5 \mathrm{~Hz}, 3 \mathrm{H}) ;{ }^{13} \mathrm{C}\left\{{ }^{1} \mathrm{H}\right\} \mathrm{NMR}\left(100 \mathrm{MHz}, \mathrm{CDCl}_{3}\right) \delta 188.6,159.4$, 158.9, 151.4, 149.6, 140.2, 133.0, 130.5, 126.2, 122.1, 104.3, 99.8, 56.0, 55.7, 18.0, 15.9. HRMS (ESI) m/z: $[\mathrm{M}+\mathrm{H}]^{+}$calcd for $\mathrm{C}_{16} \mathrm{H}_{18} \mathrm{NO}_{3} 272.1281$, found 272.1280 .

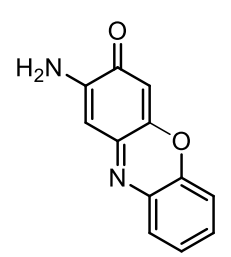

Compound 20: 2-aminophenol $(54.6 \mathrm{mg}, 0.5 \mathrm{mmol})$ were reacted according to method $\mathrm{A}$ (in this reaction 3 equiv of $t$ - $\mathrm{BuOOH}$ were added). The crude residue was purified by column chromatography (hexane/ethyl acetate 70:30) to afford compound 20 (44.1 mg, 83\% yield) as an orange red solid.

Characterization data of compound 20: ${ }^{1} \mathrm{H}$ NMR $\left(500 \mathrm{MHz}\right.$, DMSO- $\left.d_{6}, 347 \mathrm{~K}\right) \delta 7.67(\mathrm{~d}, J=8.0$ $\mathrm{Hz}, 1 \mathrm{H}), 7.46-7.41(\mathrm{~m}, 2 \mathrm{H}), 7.40-7.33(\mathrm{~m}, 1 \mathrm{H}), 6.57$ (bs, 2H), $6.40(\mathrm{~s}, 1 \mathrm{H}), 6.31(\mathrm{~s}, 1 \mathrm{H})$; ${ }^{13} \mathrm{C}\left\{{ }^{1} \mathrm{H}\right\}$ NMR $\left(125 \mathrm{MHz}, \mathrm{DMSO}-d_{6}, 347 \mathrm{~K}\right) \delta 179.7,148.5,148.0,146.9,141.7,133.4,128.4$, 127.6, 124.8, 115.4, 103.1, 98.4. HRMS (ESI) m/z: $[\mathrm{M}+\mathrm{H}]^{+}$calcd for $\mathrm{C}_{12} \mathrm{H}_{9} \mathrm{~N}_{2} \mathrm{O}_{2} 213.0659$, found 213.0658 .

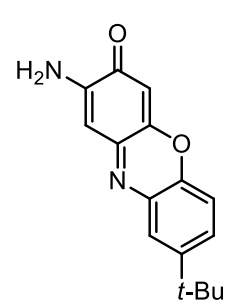

Compound 21: 2-aminophenol (27.3 mg, $0.25 \mathrm{mmol})$ and 2-amino-(4-t-Bu)phenol (123.9 $\mathrm{mg}, 0.75 \mathrm{mmol}$ ) were reacted according to method A (in this reaction 3 equiv of $t$-BuOOH were added). The crude residue was purified by column chromatography (hexane/ethyl acetate 85:15) to afford compound 21 (55.2 $\mathrm{mg}$, $82 \%$ yield) as a red solid.

Characterization data of compound 21: ${ }^{1} \mathrm{H}$ NMR (400 MHz, $\left.\mathrm{CDCl}_{3}\right) \delta 7.74(\mathrm{~d}, J=2.3 \mathrm{~Hz}, 1 \mathrm{H}), 7.47$ (dd, $J=8.7,2.3 \mathrm{~Hz}, 1 \mathrm{H}), 7.30$ (d, $J=8.7 \mathrm{~Hz}, 1 \mathrm{H}), 6.46$ (s, 1H), 6.39 (s, 1H), 5.20 (bs, 2H), 1.36 (s, $9 \mathrm{H}) ;{ }^{13} \mathrm{C}\left\{{ }^{1} \mathrm{H}\right\} \mathrm{NMR}\left(100 \mathrm{MHz}, \mathrm{CDCl}_{3}\right) \delta 180.4,149.7,148.8,148.6,145.9,140.8,133.5,127.5$, 125.3, 115.5, 104.0, 100.8, 34.8, 31.5. HRMS (ESI) m/z: $[\mathrm{M}+\mathrm{H}]^{+}$calcd for $\mathrm{C}_{16} \mathrm{H}_{17} \mathrm{~N}_{2} \mathrm{O}_{2} 269.1285$, found 269.1285 . 


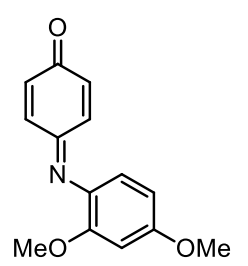

Compound 22: 4-methoxyphenol (46.6 $\mathrm{mg}, \quad 0.375 \mathrm{mmol})$ and 2,4dimethoxyaniline $(38.3 \mathrm{mg}, 0.25 \mathrm{mmol}$ ) were reacted according to method A. The crude residue was purified by column chromatography (hexane/ethyl acetate 80:20) to afford compound 22 (32.2 $\mathrm{mg}, 53 \%$ yield) as a dark pink syrup.

Characterization data of compound 22: ${ }^{1} \mathrm{H} \mathrm{NMR}\left(400 \mathrm{MHz}, \mathrm{CDCl}_{3}\right) \delta 7.37(\mathrm{dd}, J=10.1,2.7 \mathrm{~Hz}$, $1 \mathrm{H}), 7.13(\mathrm{dd}, J=10.3,2.7 \mathrm{~Hz}, 1 \mathrm{H}), 6.67-6.63(\mathrm{~m}, 2 \mathrm{H}), 6.56(\mathrm{~d}, J=2.5 \mathrm{~Hz}, 1 \mathrm{H}), 6.52-6.49$ (m, 2H), $3.84(\mathrm{~s}, 3 \mathrm{H}), 3.84(\mathrm{~s}, 3 \mathrm{H}) ;{ }^{13} \mathrm{C}\left\{{ }^{1} \mathrm{H}\right\} \mathrm{NMR}\left(100 \mathrm{MHz}, \mathrm{CDCl}_{3}\right) \delta 188.1,160.7,157.0,152.9$, 142.2, 132.7, 132.5, 132.0, 129.0, 123.1, 104.4, 99.7, 56.0, 55.7. HRMS (ESI) m/z: $[\mathrm{M}+\mathrm{H}]^{+}$calcd for $\mathrm{C}_{14} \mathrm{H}_{14} \mathrm{NO}_{3} 244.0968$, found 244.0970 .

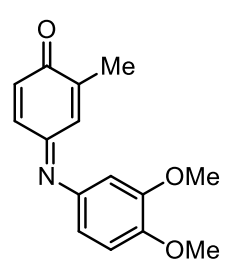

Compound 23: 4-methoxy-2-methylphenol (51.8 mg, $0.375 \mathrm{mmol}$ ) and 3,4dimethoxyaniline $(38.3 \mathrm{mg}, 0.25 \mathrm{mmol}$ ) were reacted according to method A. The crude residue was purified by column chromatography (hexane/ethyl acetate 85:15) to afford two isomeric conformations of compound $\mathbf{2 3}(47.1 \mathrm{mg}, 73 \%$ yield) as a dark red solid.

Characterization data of two isomeric conformations of compound 23: ${ }^{1} \mathrm{H} \mathrm{NMR}\left(400 \mathrm{MHz}, \mathrm{CDCl}_{3}\right)$ $\delta 7.21(\mathrm{~d}, J=2.7 \mathrm{~Hz}), 7.18(\mathrm{~d}, J=2.6 \mathrm{~Hz}), 7.14(\mathrm{~d}, J=2.7 \mathrm{~Hz}), 7.12(\mathrm{~d}, J=2.7 \mathrm{~Hz}), 7.09(\mathrm{dq}, J=$ 2.8, $1.4 \mathrm{~Hz}), 7.01(\mathrm{dq}, J=2.9,1.5 \mathrm{~Hz}), 6.88(\mathrm{~d}, J=8.5 \mathrm{~Hz}), 6.86(\mathrm{~d}, J=8.5 \mathrm{~Hz}), 6.64(\mathrm{~d}, J=9.9$ $\mathrm{Hz}), 6.58(\mathrm{~d}, J=2.4 \mathrm{~Hz}), 6.58(\mathrm{~d}, J=2.4 \mathrm{~Hz}), 6.51(\mathrm{~d}, J=10.1 \mathrm{~Hz}), 6.40(\mathrm{dd}, J=8.3,2.2 \mathrm{~Hz}), 6.38$ (dd, $J=8.3,2.3 \mathrm{~Hz}), 3.90$ (s), 3.89 (s), 3.87 (s), 3.86 (s), 2.07 (d, $J=1.4 \mathrm{~Hz}), 1.98$ (d, $J=1.5 \mathrm{~Hz}$ ); ${ }^{13} \mathrm{C}\left\{{ }^{1} \mathrm{H}\right\} \mathrm{NMR}\left(100 \mathrm{MHz}, \mathrm{CDCl}_{3}\right) \delta 188.2,188.1,157.5,157.3,149.6,148.1,147.9,143.5,143.4$, 141.8, 140.8, 138.4, 133.1, 132.4, 128.1, 125.2, 113.5, 113.1, 111.1 (x 2C), 106.6, 106.4, 56.2, 56.0, 16.5, 16.0. HRMS (ESI) m/z: $[\mathrm{M}+\mathrm{H}]^{+}$calcd for $\mathrm{C}_{15} \mathrm{H}_{16} \mathrm{NO}_{3} 258.1125$, found 258.1125.

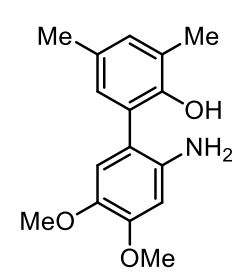

Compound 24: 2,4-dimethylphenol (45 $\mu \mathrm{L}, \quad 0.375 \quad \mathrm{mmol})$ and 3,4dimethoxyaniline $(38.3 \mathrm{mg}, 0.25 \mathrm{mmol}$ ) were reacted according to method B. The crude residue was purified by column chromatography (hexane/ethyl acetate 70:30) to afford compound $\mathbf{2 4}$ (35.3 $\mathrm{mg}, 52 \%$ yield) as a brown solid.

Characterization data of compound 24: ${ }^{1} \mathrm{H}$ NMR $\left(400 \mathrm{MHz}, \mathrm{CDCl}_{3}\right) \delta 7.00-6.98(\mathrm{~m}, 1 \mathrm{H}), 6.93$ (dd, $J=1.6,0.6 \mathrm{~Hz}, 1 \mathrm{H}), 6.76(\mathrm{~s}, 1 \mathrm{H}), 6.43(\mathrm{~s}, 1 \mathrm{H}), 3.88$ (s, 3H), 3.84 (s, 3H), 2.30 (s, 3H), 2.30 (s, $3 \mathrm{H}) ;{ }^{13} \mathrm{C}\left\{{ }^{1} \mathrm{H}\right\} \mathrm{NMR}\left(100 \mathrm{MHz}, \mathrm{CDCl}_{3}\right) \delta 149.7,149.6,143.6,135.4,131.2,129.7,128.9,126.8$, 125.5, 118.1, 115.1, 102.1, 56.5, 56.1, 20.6, 16.6. HRMS (ESI) m/z: $[\mathrm{M}+\mathrm{H}]^{+}$calcd for $\mathrm{C}_{16} \mathrm{H}_{20} \mathrm{NO}_{3}$ 274.1438 , found 274.1432 . 


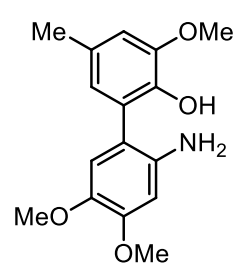

Compound 25: 2-methoxy-4-methylphenol (48 $\mu \mathrm{L}, 0.375 \mathrm{mmol}$ ) and 3,4dimethoxyaniline $(38.3 \mathrm{mg}, 0.25 \mathrm{mmol})$ were reacted according to method $\mathrm{B}$. The crude residue was purified by column chromatography (hexane/ethyl acetate 50:50) to afford compound 25 (33.6 $\mathrm{mg}, 46 \%$ yield) as a brown solid.

Characterization data of compound 25: ${ }^{1} \mathrm{H}$ NMR $\left(400 \mathrm{MHz}, \mathrm{CDCl}_{3}\right) \delta 6.75(\mathrm{~s}, 1 \mathrm{H}), 6.71(\mathrm{~d}, J=1.7$ Hz, 1H), 6.69 (dd, $J=1.8,0.6 \mathrm{~Hz}, 1 \mathrm{H}), 6.41$ (s, 1H), 3.90 (s, 3H), 3.86 (s, 3H), 3.82 (s, 3H), 2.33 (s, $3 \mathrm{H}) ;{ }^{13} \mathrm{C}\left\{{ }^{1} \mathrm{H}\right\} \mathrm{NMR}\left(100 \mathrm{MHz}, \mathrm{CDCl}_{3}\right) \delta 149.5,147.9,142.9,140.7,136.6,129.9,125.8,123.5$, 117.1, 114.9, 111.3, 101.8, 56.6, 56.1, 56.0, 21.2. HRMS (ESI) m/z: $[\mathrm{M}+\mathrm{H}]^{+}$calcd for $\mathrm{C}_{16} \mathrm{H}_{20} \mathrm{NO}_{4}$ 290.1387 , found 290.1387 .

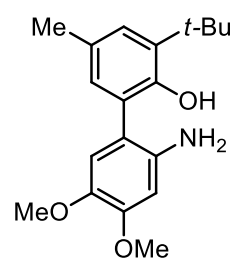

Compound 26: 2-t-Butyl-4-methylphenol (61.6 mg, $0.375 \mathrm{mmol}$ ) and 3,4dimethoxyaniline $(38.3 \mathrm{mg}, 0.25 \mathrm{mmol}$ ) were reacted according to method $\mathrm{B}$. The crude residue was purified by column chromatography (hexane/ethyl acetate 80:20) to afford compound $\mathbf{2 6}$ (56.3 $\mathrm{mg}, 71 \%$ yield) as a brown solid.

Characterization data of compound 26: ${ }^{1} \mathrm{H}$ NMR $\left(400 \mathrm{MHz}, \mathrm{CDCl}_{3}\right) \delta 7.13(\mathrm{~d}, J=2.1 \mathrm{~Hz}, 1 \mathrm{H}), 6.96$ $(\mathrm{dd}, J=2.2,0.7 \mathrm{~Hz}, 1 \mathrm{H}), 6.76(\mathrm{~s}, 1 \mathrm{H}), 6.43(\mathrm{~s}, 1 \mathrm{H}), 3.88(\mathrm{~s}, 3 \mathrm{H}), 3.83$ (s, 3H), 2.34 (s, 3H), 1.46 (s, $9 \mathrm{H}) ;{ }^{13} \mathrm{C}\left\{{ }^{1} \mathrm{H}\right\} \mathrm{NMR}\left(100 \mathrm{MHz}, \mathrm{CDCl}_{3}\right) \delta 150.2,149.7,143.5,138.1,135.9,129.2$ (x 2C), 127.4, 126.7, 117.4, 115.3, 101.8, 56.5, 56.1, 35.0, 29.9, 21.0. HRMS (ESI) m/z: $[\mathrm{M}]^{+}$calcd for $\mathrm{C}_{19} \mathrm{H}_{25} \mathrm{NO}_{3} 315.1829$, found 315.1828 .

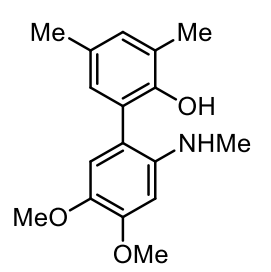

Compound 27: 2,4-dimethylphenol (45 $\mu \mathrm{L}, 0.375 \mathrm{mmol}$ ) and $N$-methyl-3,4dimethoxyaniline $(41.8 \mathrm{mg}, 0.25 \mathrm{mmol}$ ) were reacted according to method $\mathrm{B}$. The crude residue was purified by column chromatography (hexane/ethyl acetate $85: 15)$ to afford compound 27 (13 $\mathrm{mg}, 18 \%$ yield) as a brown red solid.

Characterization data of compound 27: ${ }^{1} \mathrm{H}$ NMR $\left(400 \mathrm{MHz}, \mathrm{CDCl}_{3}\right) \delta 7.00-6.98(\mathrm{~m}, 1 \mathrm{H}), 6.91$ (dd, $J=1.6,0.6 \mathrm{~Hz}, 1 \mathrm{H}), 6.80(\mathrm{~s}, 1 \mathrm{H}), 6.47(\mathrm{~s}, 1 \mathrm{H}), 3.95(\mathrm{~s}, 3 \mathrm{H}), 3.84(\mathrm{~s}, 3 \mathrm{H}), 2.81(\mathrm{~s}, 3 \mathrm{H}), 2.30$ (s, $3 \mathrm{H}), 2.29(\mathrm{~s}, 3 \mathrm{H}) ;{ }^{13} \mathrm{C}\left\{{ }^{1} \mathrm{H}\right\} \mathrm{NMR}\left(100 \mathrm{MHz}, \mathrm{CDCl}_{3}\right) \delta 149.9,149.8,142.8,139.4,131.2,129.7$, 129.1, 126.5, 125.3, 118.1, 115.8, 98.2, 56.7, 56.2, 31.8, 20.7, 16.6. HRMS (ESI) m/z: [M] ${ }^{+}$calcd for $\mathrm{C}_{17} \mathrm{H}_{21} \mathrm{NO}_{3} 287.1516$, found 287.1518.

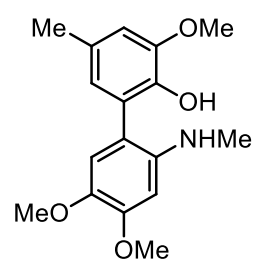

Compound 28: 2-methoxy-4-methylphenol (48 $\mu \mathrm{L}, 0.375 \mathrm{mmol})$ and $N$-methyl3,4-dimethoxyaniline $(41.8 \mathrm{mg}, 0.25 \mathrm{mmol})$ were reacted according to method $\mathrm{B}$. The crude residue was purified by column chromatography (hexane/ethyl acetate 60:40) to afford compound $\mathbf{2 8}(15.7 \mathrm{mg}, 21 \%$ yield) as a brown red solid. 
Characterization data of compound 28: ${ }^{1} \mathrm{H}$ NMR $\left(400 \mathrm{MHz}, \mathrm{CDCl}_{3}\right) \delta 6.79(\mathrm{~s}, 1 \mathrm{H}), 6.72(\mathrm{~d}, J=1.9$ Hz, 1H), 6.69 (dd, J = 1.4, 0.4 Hz, 1H), 6.46 (s, 1H), 3.94 (s, 3H), 3.91 (s, 3H), 3.83 (s, 3H), 2.81 (s, $3 \mathrm{H}), 2.33(\mathrm{~s}, 3 \mathrm{H}) ;{ }^{13} \mathrm{C}\left\{{ }^{1} \mathrm{H}\right\} \mathrm{NMR}\left(100 \mathrm{MHz}, \mathrm{CDCl}_{3}\right) \delta 149.8,148.1,142.3,141.0,139.9,130.0$, 125.8, 123.5, 117.5, 115.6, 111.5, 98.0, 56.8, 56.1, 31.9, 21.3. HRMS (ESI) m/z: $[\mathrm{M}+\mathrm{H}]^{+}$calcd for $\mathrm{C}_{17} \mathrm{H}_{22} \mathrm{NO}_{4} 304.1543$, found 304.1533.

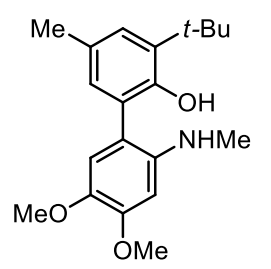

Compound 29: 2-t-Butyl-4-methylphenol (61.6 mg, $0.375 \mathrm{mmol})$ and $N$-methyl3,4-dimethoxyaniline $(41.8 \mathrm{mg}, 0.25 \mathrm{mmol})$ were reacted according to method $\mathrm{B}$. The crude residue was purified by column chromatography (hexane/ethyl acetate 90:10) to afford compound $\mathbf{2 9}(31.2 \mathrm{mg}, 38 \%$ yield) as a brown red solid.

Characterization data of compound 29: ${ }^{1} \mathrm{H}$ NMR (400 MHz, $\left.\mathrm{CDCl}_{3}\right) \delta 7.13(\mathrm{~d}, J=2.3 \mathrm{~Hz}, 1 \mathrm{H}), 6.95$ $(\mathrm{dd}, J=2.2,0.7 \mathrm{~Hz}, 1 \mathrm{H}), 6.81(\mathrm{~s}, 1 \mathrm{H}), 6.46(\mathrm{~s}, 1 \mathrm{H}), 3.95$ (s, 3H), 3.85 (s, 3H), $2.81(\mathrm{~s}, 3 \mathrm{H}), 2.33$ (s, $3 \mathrm{H}), 1.46(\mathrm{~s}, 9 \mathrm{H}) ;{ }^{13} \mathrm{C}\left\{{ }^{1} \mathrm{H}\right\} \mathrm{NMR}\left(100 \mathrm{MHz}, \mathrm{CDCl}_{3}\right) \delta 150.5,149.9,142.6,139.9,137.8,129.4$, 129.2, 127.4, 126.5, 117.5, 116.0, 98.0, 56.7, 56.2, 35.0, 31.8, 29.9, 21.0. HRMS (ESI) m/z: $[\mathrm{M}+\mathrm{H}]^{+}$calcd for $\mathrm{C}_{20} \mathrm{H}_{28} \mathrm{NO}_{3} 330.2064$, found 330.2068.

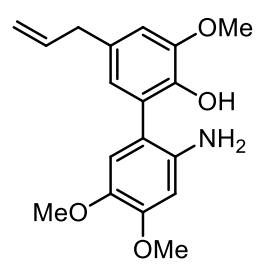

Compound 30: Eugenol $(58 \mu \mathrm{L}, 0.375 \mathrm{mmol})$ and 3,4-dimethoxyaniline (38.3 $\mathrm{mg}, 0.25 \mathrm{mmol}$ ) were reacted according to method $\mathrm{B}$. The crude residue was purified by column chromatography (hexane/ethyl acetate 50:50) to afford compound 30 (22.4 mg, $28 \%$ yield) as a brown solid.

Characterization data of compound 30: ${ }^{1} \mathrm{H}$ NMR $\left(400 \mathrm{MHz}, \mathrm{CDCl}_{3}\right) \delta 6.75(\mathrm{~s}, 1 \mathrm{H}), 6.72(\mathrm{~s}, 2 \mathrm{H})$, $6.41(\mathrm{~s}, 1 \mathrm{H}), 5.98(\mathrm{ddt}, J=16.8,10.0,6.8 \mathrm{~Hz}, 1 \mathrm{H}), 5.14-5.05(\mathrm{~m}, 2 \mathrm{H}), 3.91(\mathrm{~s}, 3 \mathrm{H}), 3.86(\mathrm{~s}, 3 \mathrm{H})$, $3.82(\mathrm{~s}, 3 \mathrm{H}), 3.36(\mathrm{~d}, J=6.7 \mathrm{~Hz}, 2 \mathrm{H}) ;{ }^{13} \mathrm{C}\left\{{ }^{1} \mathrm{H}\right\} \mathrm{NMR}\left(100 \mathrm{MHz}, \mathrm{CDCl}_{3}\right) \delta 149.6,148.0,143.0$, 141.3, 137.7, 136.6, 132.2, 125.9, 123.1, 117.0, 115.9, 115.0, 110.6, 101.8, 56.6, 56.2, 56.0, 40.1. HRMS (ESI) m/z: $[\mathrm{M}+\mathrm{H}]^{+}$calcd for $\mathrm{C}_{18} \mathrm{H}_{22} \mathrm{NO}_{4} 316.1543$, found 316.1533 .

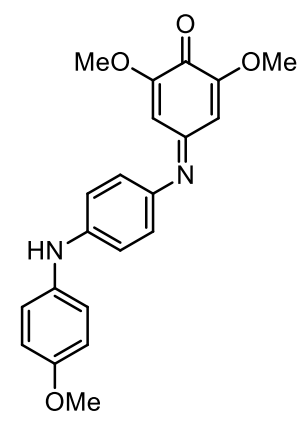

Compound 31: 2,6-dimethoxyphenol $(57.8 \mathrm{mg}, 0.375 \mathrm{mmol})$ and 4-(4methoxyphenylamino)aniline $(53.6 \mathrm{mg}, 0.25 \mathrm{mmol})$ were reacted according to method A. The crude residue was purified by column chromatography (hexane/ethyl acetate 50:50) to afford compound $\mathbf{3 1}$ (72.4 $\mathrm{mg}, 79 \%$ yield) as a dark blue solid.

Characterization data of compound 31: ${ }^{1} \mathrm{H}$ NMR $\left(400 \mathrm{MHz}, \mathrm{CDCl}_{3}\right) \delta 7.13-$ $7.07(\mathrm{~m}, 2 \mathrm{H}), 6.96-6.91(\mathrm{~m}, 2 \mathrm{H}), 6.90-6.84(\mathrm{~m}, 4 \mathrm{H}), 6.42(\mathrm{~d}, J=2.2 \mathrm{~Hz}$, $1 \mathrm{H}), 6.37(\mathrm{~d}, J=2.2 \mathrm{~Hz}, 1 \mathrm{H}), 5.80(\mathrm{bs}, 1 \mathrm{H}), 3.82(\mathrm{~s}, 3 \mathrm{H}), 3.79(\mathrm{~s}, 3 \mathrm{H}), 3.70(\mathrm{~s}, 3 \mathrm{H}) ;{ }^{13} \mathrm{C}\left\{{ }^{1} \mathrm{H}\right\} \mathrm{NMR}$ $\left(100 \mathrm{MHz} \mathrm{CDCl}_{3}\right) \delta 176.6,155.8,155.4,155.3,154.3,144.0,142.4,134.9,124.0,122.9,115.5$, 
114.8, 112.6, 99.2, 56.1, 56.0, 55.6. HRMS (ESI) m/z: $[\mathrm{M}+\mathrm{H}]^{+}$calcd for $\mathrm{C}_{21} \mathrm{H}_{21} \mathrm{~N}_{2} \mathrm{O}_{4} 365.1496$, found 365.1494 .

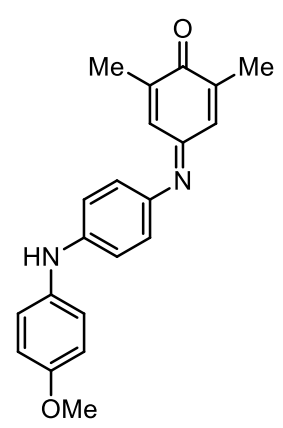

Compound 32: 2,6-dimethylphenol (45.8 mg, $0.375 \mathrm{mmol})$ and 4-(4methoxyphenylamino)aniline $(53.6 \mathrm{mg}, 0.25 \mathrm{mmol})$ were reacted according to method A. The crude residue was purified by column chromatography (hexane/ethyl acetate 85:15) to afford compound $\mathbf{3 2}$ (73.4 $\mathrm{mg}, 88 \%$ yield) as a dark blue solid.

Characterization data of compound 32: ${ }^{1} \mathrm{H} \mathrm{NMR}\left(400 \mathrm{MHz}, \mathrm{CDCl}_{3}\right) \delta 7.14-$ $7.09(\mathrm{~m}, 2 \mathrm{H}), 7.08-7.04(\mathrm{~m}, 2 \mathrm{H}), 6.95-6.91(\mathrm{~m}, 2 \mathrm{H}), 6.91-6.85(\mathrm{~m}, 4 \mathrm{H})$,

$5.82(\mathrm{bs}, 1 \mathrm{H}), 3.80(\mathrm{~s}, 3 \mathrm{H}), 2.08(\mathrm{~d}, J=1.3 \mathrm{~Hz}, 3 \mathrm{H}), 2.01(\mathrm{~d}, J=1.4 \mathrm{~Hz}, 3 \mathrm{H}) ;{ }^{13} \mathrm{C}\left\{{ }^{1} \mathrm{H}\right\} \mathrm{NMR}(100$ $\left.\mathrm{MHz}_{2} \mathrm{CDCl}_{3}\right) \delta 188.5,156.1,155.9,144.5,142.1,141.0,139.9,138.6,134.8,125.0,124.5,123.0$, 115.4, 114.9, 55.7, 16.8, 16.2. HRMS (ESI) m/z: $[\mathrm{M}+\mathrm{H}]^{+}$calcd for $\mathrm{C}_{21} \mathrm{H}_{21} \mathrm{~N}_{2} \mathrm{O}_{2} 333.1598$, found 333.1610 .

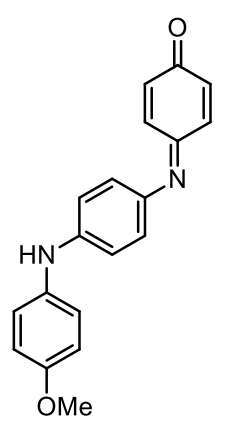

Compound 33: 4-methoxyphenol (46.6 $\mathrm{mg}, \quad 0.375 \mathrm{mmol})$ and 4-(4methoxyphenylamino)aniline $(53.6 \mathrm{mg}, 0.25 \mathrm{mmol})$ were reacted according to method A. The crude residue was purified by column chromatography (hexane/ethyl acetate 80:20) to afford compound $\mathbf{3 3}$ (35.6 $\mathrm{mg}, 47 \%$ yield) as a dark blue solid.

Characterization data of compound 33: ${ }^{1} \mathrm{H} \mathrm{NMR}\left(400 \mathrm{MHz}, \mathrm{CDCl}_{3}\right) \delta 7.31$ (q, $J$ $=2.4 \mathrm{~Hz}, 1 \mathrm{H}), 7.29(\mathrm{q}, J=2.4 \mathrm{~Hz}, 1 \mathrm{H}), 7.13(\mathrm{~d}, J=8.8 \mathrm{~Hz}, 2 \mathrm{H}), 6.98-6.85(\mathrm{~m}$,

$6 \mathrm{H}), 6.65(\mathrm{dd}, J=10.2,2.2 \mathrm{~Hz}, 1 \mathrm{H}), 6.55(\mathrm{dd}, J=10.4,2.2 \mathrm{~Hz}, 1 \mathrm{H}), 5.94(\mathrm{bs}, 1 \mathrm{H}), 3.81(\mathrm{~s}, 3 \mathrm{H})$; ${ }^{13} \mathrm{C}\left\{{ }^{1} \mathrm{H}\right\} \mathrm{NMR}\left(100 \mathrm{MHz}, \mathrm{CDCl}_{3}\right) \delta 188.1,156.3,155.0,145.9,142.4,141.7,134.1,132.5,131.7$, 128.6, 125.5, 123.6, 115.1, 114.9, 55.7. HRMS (ESI) m/z: $[\mathrm{M}+\mathrm{H}]^{+}$calcd for $\mathrm{C}_{19} \mathrm{H}_{17} \mathrm{~N}_{2} \mathrm{O}_{2} 305.1285$, found 305.1290 .

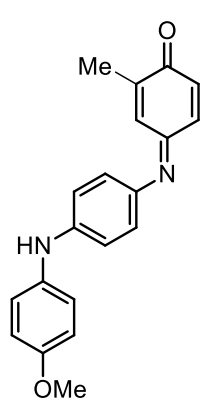

Compound 34: 4-methoxy-2-methylphenol (51.8 mg, $0.375 \mathrm{mmol})$ and 4-(4methoxyphenylamino)aniline $(53.6 \mathrm{mg}, 0.25 \mathrm{mmol})$ were reacted according to method A. The crude residue was purified by column chromatography (hexane/ethyl acetate 85:15) to afford two isomeric conformations of compound 34 (49.8 mg, 63\% yield) as a dark blue solid.

Characterization data of two isomeric conformations of compound 34: ${ }^{1} \mathrm{H}$ NMR $\left(400 \mathrm{MHz}, \mathrm{CDCl}_{3}\right) \delta 7.23(\mathrm{dd}, J=2.6,1.8 \mathrm{~Hz}), 7.21(\mathrm{dd}, J=2.6,1.4 \mathrm{~Hz}), 7.14-$ $7.08(\mathrm{~m}), 6.95-6.86(\mathrm{~m}), 6.63(\mathrm{~d}, J=9.9 \mathrm{~Hz}), 6.53(\mathrm{~d}, J=10.1 \mathrm{~Hz}), 5.94(\mathrm{bs}), 3.80$ (s), 3.80 (s), 
$2.07(\mathrm{~d}, J=1.4 \mathrm{~Hz}), 2.01(\mathrm{~d}, J=1.4 \mathrm{~Hz}) ;{ }^{13} \mathrm{C}\left\{{ }^{1} \mathrm{H}\right\} \mathrm{NMR}\left(100 \mathrm{MHz}, \mathrm{CDCl}_{3}\right) \delta 188.3$ (x 2C), 156.1, 155.8, 155.7, 145.3, 145.2, 142.1, 141.8 (x 2C), 141.0, 140.0, 138.8, 134.4, 132.4, 131.6, 128.1, 125.4, 125.1, 124.9, 123.3 (x 2C), 115.2 (x 2C), 114.9, 55.6, 16.6, 16.0. HRMS (ESI) m/z: [M+H] ${ }^{+}$ calcd for $\mathrm{C}_{20} \mathrm{H}_{19} \mathrm{~N}_{2} \mathrm{O}_{2} 319.1441$, found 319.1442 .

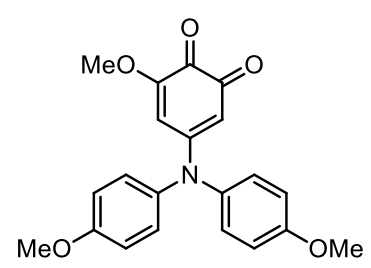

Compound 35: 2,6-dimethoxyphenol (57.8 $\mathrm{mg}, 0.375 \mathrm{mmol})$ and 4,4'dimethoxydiphenylamine $(57.3 \mathrm{mg}, 0.25 \mathrm{mmol})$ were reacted according to method $\mathrm{A}$ (in this reaction 4 equiv of $t$ - $\mathrm{BuOOH}$ were added). The crude residue was purified by column chromatography (hexane/ethyl acetate 30:70) to afford compound $\mathbf{3 5}$ (57.7 $\mathrm{mg}, 63 \%$ yield) as a dark grey solid.

Characterization data of compound 35: ${ }^{1} \mathrm{H} \mathrm{NMR}\left(400 \mathrm{MHz}, \mathrm{CDCl}_{3}\right) \delta 7.17-7.08(\mathrm{~m}, 4 \mathrm{H}), 6.89$ (d, $J=8.9 \mathrm{~Hz}, 4 \mathrm{H}), 5.72(\mathrm{~d}, J=2.6 \mathrm{~Hz}, 1 \mathrm{H}), 5.44(\mathrm{~d}, J=2.6 \mathrm{~Hz}, 1 \mathrm{H}), 3.80(\mathrm{~s}, 6 \mathrm{H}), 3.55(\mathrm{~s}, 3 \mathrm{H})$;

${ }^{13} \mathrm{C}\left\{{ }^{1} \mathrm{H}\right\} \mathrm{NMR}\left(100 \mathrm{MHz}, \mathrm{CDCl}_{3}\right) \delta 178.6,174.1,159.1,158.7,153.4,136.0,128.7,115.0,104.4$, 100.8, 55.8, 55.7. HRMS (ESI) m/z: $[\mathrm{M}+\mathrm{H}]^{+}$calcd for $\mathrm{C}_{21} \mathrm{H}_{20} \mathrm{NO}_{5} 366.1336$, found 366.1335.

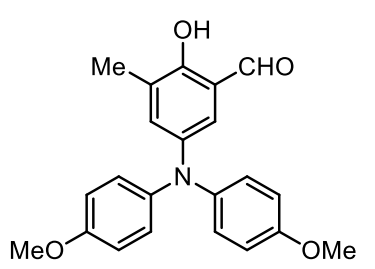

Compound 36: 2,6-dimethylphenol (45.8 mg, $0.375 \mathrm{mmol}$ ) and 4,4'dimethoxydiphenylamine $(57.3 \mathrm{mg}, 0.25 \mathrm{mmol})$ were reacted according to method $\mathrm{A}$ (in this reaction 4 equiv of $t$ - $\mathrm{BuOOH}$ were added). The crude residue was purified by column chromatography (hexane/ethyl acetate 98:2) to afford compound $\mathbf{3 6}$ (39.4 $\mathrm{mg}, 43 \%$ yield) as an orange solid.

Characterization data of compound 36: ${ }^{1} \mathrm{H}$ NMR $\left(400 \mathrm{MHz}, \mathrm{CDCl}_{3}\right) \delta 11.00(\mathrm{~s}, 1 \mathrm{H}), 9.69(\mathrm{~s}, 1 \mathrm{H})$, $7.15(\mathrm{dt}, J=2.8,0.7 \mathrm{~Hz}, 1 \mathrm{H}), 7.01(\mathrm{dd}, J=2.8,0.6 \mathrm{~Hz}, 1 \mathrm{H}), 7.00-6.93(\mathrm{~m}, 4 \mathrm{H}), 6.85-6.77$ (m, 4H), $3.79(\mathrm{~s}, 6 \mathrm{H}), 2.19(\mathrm{~s}, 3 \mathrm{H}) ;{ }^{13} \mathrm{C}\left\{{ }^{1} \mathrm{H}\right\} \mathrm{NMR}\left(100 \mathrm{MHz}, \mathrm{CDCl}_{3}\right) \delta$ 196.6, 155.5, 155.4, 141.5, 141.0, 134.0, 128.0, 125.2, 124.6, 120.3, 114.8, 55.6, 15.3. HRMS (ESI) m/z: $[\mathrm{M}-\mathrm{H}]^{+}$calcd for $\mathrm{C}_{22} \mathrm{H}_{20} \mathrm{NO}_{4} 362.1387$, found 362.1386 . 


\subsection{Synthesis of compounds 13a, 37-38:}

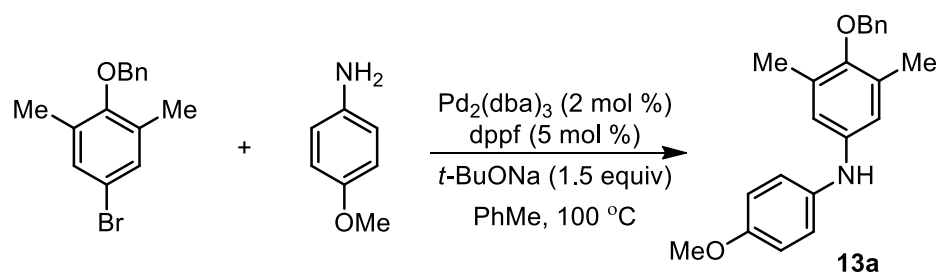

2.2.1 Compound 13a: A solution of 2-benzyloxy-5-bromo-1,3-dimethylbenzene (582 mg, 2 mmol), 4-methoxyaniline (370 mg, $3 \mathrm{mmol}), \mathrm{Pd}_{2}(\mathrm{dba})_{3}(37 \mathrm{mg}, 2 \mathrm{~mol} \%$ ), dppf (55 mg, $5 \mathrm{~mol} \%$ ) and $t$-BuONa $(288 \mathrm{mg}, 3 \mathrm{mmol})$ in toluene $(7 \mathrm{ml})$ was stirred at $100{ }^{\circ} \mathrm{C}$ (oil bath) overnight under argon. After cooling to room temperature, the volatiles were removed under reduced pressure and the crude was purified by silica-gel column chromatography (hexane/ethyl acetate 95:5) to afford compound 13a (624 mg, 94\% yield) as a gray syrup.

Characterization data of compound 13a: ${ }^{1} \mathrm{H}$ NMR $\left(400 \mathrm{MHz}, \mathrm{CDCl}_{3}\right) \delta 7.53-7.49(\mathrm{~m}, 2 \mathrm{H}), 7.41$ (tt, $J=7.9,1.7 \mathrm{~Hz}, 2 \mathrm{H}), 7.38-7.33(\mathrm{~m}, 1 \mathrm{H}), 7.07-7.00(\mathrm{~m}, 2 \mathrm{H}), 6.90-6.84(\mathrm{~m}, 2 \mathrm{H}), 6.63(\mathrm{~s}, 2 \mathrm{H})$, 5.32 (bs, 1H), 4.79 (s, 2H), $3.81(\mathrm{~s}, 3 \mathrm{H}), 2.26(\mathrm{~s}, 6 \mathrm{H}) ;{ }^{13} \mathrm{C}\left\{{ }^{1} \mathrm{H}\right\} \mathrm{NMR}\left(100 \mathrm{MHz}, \mathrm{CDCl}_{3}\right) \delta 154.9$, 149.5, 140.9, 138.0, 136.8, 132.0, 128.6, 128.0, 127.9, 121.4, 116.7, 114.8, 74.4, 55.7, 16.7. HRMS (ESI) $\mathrm{m} / \mathrm{z}:[\mathrm{M}+\mathrm{H}]^{+}$calcd for $\mathrm{C}_{22} \mathrm{H}_{24} \mathrm{NO}_{2} 334.1802$, found 334.1797 .

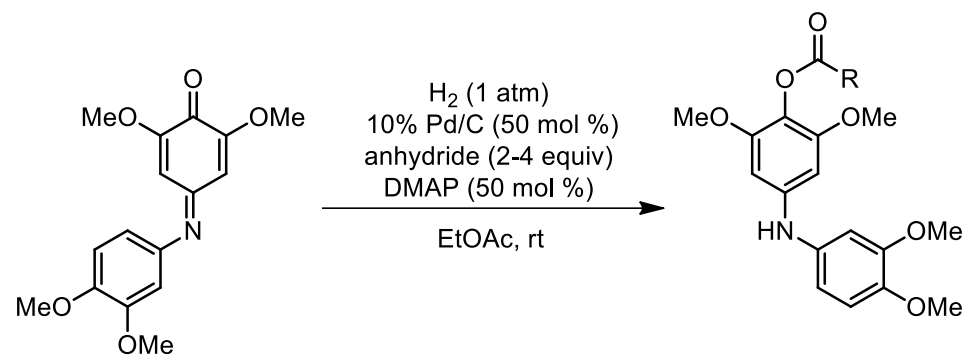

2.2.2 General procedure: To an oven-dried round bottom flask equipped with a nitrogen balloon was added $10 \% \mathrm{Pd} / \mathrm{C}(50 \mathrm{~mol} \%)$, followed by the addition of EtOAc $(0.05 \mathrm{M})$, benzoquinone anils 3 ( 1 equiv), anhydride (2-4 equiv) and 4-dimethylaminopyridine (50 mol \%). The nitrogen balloon was replaced with hydrogen balloon, purged three times and then the reaction was stirred for $2 \mathrm{~h}$ at room temperature. The suspended $\mathrm{Pd} / \mathrm{C}$ was removed by filtration through celite and the filtrate was evaporated under reduced pressure. The crude was further purified by silica-gel column chromatography (silica gel 40-63 $\mu \mathrm{m}$ ). 


\subsubsection{Characterization data:}<smiles>COC(=O)Oc1c(OC)cc(Nc2ccc(OC)cc2)cc1OC</smiles>

Compound 37: The Compound was prepared from benzoquinone anils 3 (30.3 $\mathrm{mg}, 0.1 \mathrm{mmol}$ ) according to the general procedure (in this reaction 2 equiv of Boc anhydride were added). The crude residue was purified by silica-gel column chromatography (hexane/ethyl acetate 70:30) to afford compound 37 (41 $\mathrm{mg}, 99 \%$ yield) as a pale brown solid.

Characterization data of compound 37: ${ }^{1} \mathrm{H} \mathrm{NMR}\left(400 \mathrm{MHz}, \mathrm{CDCl}_{3}\right) \delta 6.80(\mathrm{~d}, J=8.5 \mathrm{~Hz}, 1 \mathrm{H}), 6.71$ $(\mathrm{d}, J=2.5 \mathrm{~Hz}, 1 \mathrm{H}), 6.63(\mathrm{dd}, J=8.5,2.4 \mathrm{~Hz}, 1 \mathrm{H}), 6.15(\mathrm{~s}, 2 \mathrm{H}), 5.59$ (bs, 1H), 3.85 (s, 3H), 3.81 (s, $3 \mathrm{H}), 3.73(\mathrm{~s}, 6 \mathrm{H}), 1.54(\mathrm{~s}, 9 \mathrm{H}) ;{ }^{13} \mathrm{C}\left\{{ }^{1} \mathrm{H}\right\} \mathrm{NMR}\left(100 \mathrm{MHz}, \mathrm{CDCl}_{3}\right) \delta 153.0,152.1,149.7,144.8$, 143.6, 136.1, 122.7, 112.5, 112.3, 105.6, 93.3, 83.1, 56.3, 56.1, 56.0, 27.7. HRMS (ESI) m/z: $[\mathrm{M}+\mathrm{H}]^{+}$calcd for $\mathrm{C}_{21} \mathrm{H}_{28} \mathrm{NO}_{7} 406.1860$, found 406.1862 .

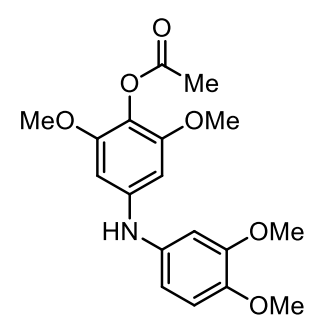

Compound 38: The compound was prepared from benzoquinone anils 3 (30.3 $\mathrm{mg}, 0.1 \mathrm{mmol}$ ) according to the general procedure (in this reaction 4 equiv of acetic anhydride were added). The crude residue was purified by silica-gel column chromatography (hexane/ethyl acetate 70:30) to afford compound $\mathbf{3 8}$ (35 mg, 99\% yield) as a pale brown solid.

Characterization data of compound 38: ${ }^{1} \mathrm{H}$ NMR $\left(400 \mathrm{MHz}, \mathrm{CDCl}_{3}\right) \delta 6.80(\mathrm{~d}, J=8.6 \mathrm{~Hz}, 1 \mathrm{H}), 6.72$ $(\mathrm{d}, J=2.5 \mathrm{~Hz}, 1 \mathrm{H}), 6.64(\mathrm{dd}, J=8.4,2.2 \mathrm{~Hz}, 1 \mathrm{H}), 6.16$ (s, 2H), $5.62(\mathrm{bs}, 1 \mathrm{H}), 3.86(\mathrm{~s}, 3 \mathrm{H}), 3.81$ (s, $3 \mathrm{H}), 3.71(\mathrm{~s}, 6 \mathrm{H}), 2.30(\mathrm{~s}, 3 \mathrm{H}) ;{ }^{13} \mathrm{C}\left\{{ }^{1} \mathrm{H}\right\} \mathrm{NMR}\left(100 \mathrm{MHz}, \mathrm{CDCl}_{3}\right) \delta$ 169.6, 152.8, 149.7, 144.9, $143.8,136.0,121.9,112.7,112.3,105.7,93.1,56.3,56.1,56.0,20.5$. HRMS (ESI) m/z: $[\mathrm{M}+\mathrm{H}]^{+}$ calcd for $\mathrm{C}_{18} \mathrm{H}_{22} \mathrm{NO}_{6} 348.1442$, found 348.1433. 


\subsection{Control experiment in Scheme 2A:}

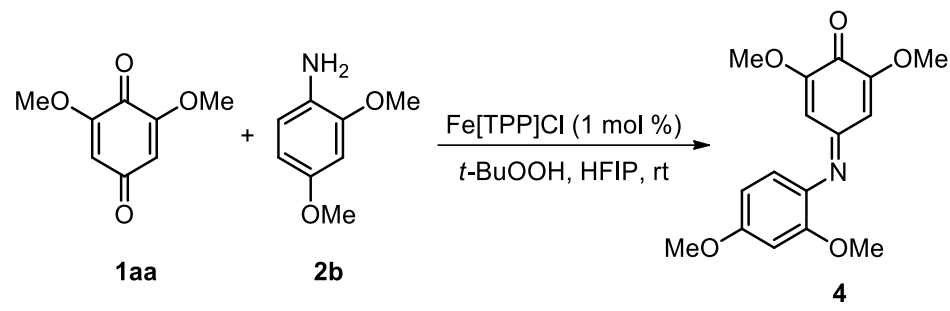

To a stirred solution of 2,6-dimethoxy-1,4-benzoquinone (63.1 $\mathrm{mg}, 0.375 \mathrm{mmol}), 2,4-$ dimethoxyaniline $(38.3 \mathrm{mg}, 0.25 \mathrm{mmol})$ and Fe[TPP]Cl $(1.7 \mathrm{mg}, 1 \mathrm{~mol} \%)$ in HFIP (500 $\mu \mathrm{L}, 0.5$ $\mathrm{M}), t$-BuOOH $(91 \mu \mathrm{L}, 0.5 \mathrm{mmol}, 5.5 \mathrm{M}$ solution in decane) was added drop-wise at room temperature. After the reaction was stirred for overnight, benzoquinone anil $\mathbf{4}$ was not observed by the HPLC. 


\section{4 ${ }^{1} \mathrm{H}-\mathrm{NMR}$ binding experiments of anilines to Fe[TPP]Cl:}

\subsubsection{General procedures:}

$$
\mathrm{Fe}[\mathrm{TPP}] \mathrm{Cl}+\underset{\text { Aniline } 2}{\text { (7 equiv) }} \stackrel{\mathrm{Ag}_{2} \mathrm{CO}_{3}(9 \text { equiv })}{\mathrm{CDCl}_{3}}(\mathrm{Fe}[\mathrm{TPP}])_{2} \mathrm{O}+\text { Aniline } 2
$$

Method A. A solution of aniline 2 (7 equiv), $\mathrm{Fe}[\mathrm{TPP}] \mathrm{Cl}(1.7 \mathrm{mg}, 2.5 \mu \mathrm{mol})$ and $\mathrm{Ag}_{2} \mathrm{CO}_{3}(6.0 \mathrm{mg}, 9$ equiv) in $\mathrm{CDCl}_{3}(600 \mu \mathrm{L})$ was stirred at room temperature for $24 \mathrm{~h}$ before the ${ }^{1} \mathrm{H}-\mathrm{NMR}$ spectrum was recorded on Bruker DPX400.

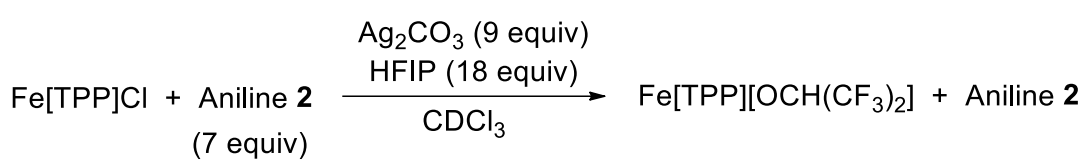

Method B. A solution of aniline 2 (7 equiv), $\mathrm{Fe}[\mathrm{TPP}] \mathrm{Cl}(1.7 \mathrm{mg}, 2.5 \mu \mathrm{mol})$ and $\mathrm{Ag}_{2} \mathrm{CO}_{3}(6.0 \mathrm{mg}, 9$ equiv) and HFIP (5 $\mu \mathrm{L}, 18$ equiv) in $\mathrm{CDCl}_{3}(600 \mu \mathrm{L})$ was stirred at room temperature for $24 \mathrm{~h}$ before the ${ }^{1} \mathrm{H}-\mathrm{NMR}$ spectrum was recorded on Bruker DPX400.

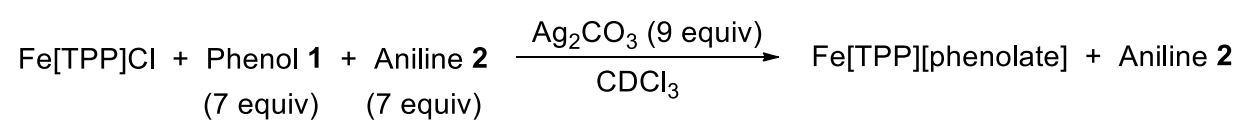

Method C. A solution of phenol 1 (7 equiv), aniline 2 (7 equiv), $\mathrm{Fe}[\mathrm{TPP}] \mathrm{Cl}(1.7 \mathrm{mg}, 2.5 \mu \mathrm{mol})$ and $\mathrm{Ag}_{2} \mathrm{CO}_{3}\left(6.0 \mathrm{mg}, 9\right.$ equiv) in $\mathrm{CDCl}_{3}(600 \mu \mathrm{L})$ was stirred at room temperature for $24 \mathrm{~h}$ before the ${ }^{1} \mathrm{H}-\mathrm{NMR}$ spectrum was recorded on Bruker DPX400.

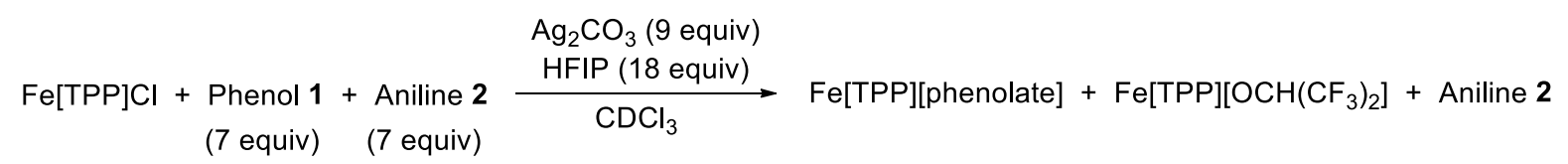

Method D. A solution of phenol 1 (7 equiv), aniline 2 (7 equiv), $\mathrm{Fe}[\mathrm{TPP}] \mathrm{Cl}(1.7 \mathrm{mg}, 2.5 \mu \mathrm{mol})$ and $\mathrm{Ag}_{2} \mathrm{CO}_{3}\left(6.0 \mathrm{mg}, 9\right.$ equiv) and $\operatorname{HFIP}\left(5 \mu \mathrm{L}, 18\right.$ equiv) in $\mathrm{CDCl}_{3}(600 \mu \mathrm{L})$ was stirred at room temperature for $24 \mathrm{~h}$ before the ${ }^{1} \mathrm{H}-\mathrm{NMR}$ spectrum was recorded on Bruker DPX400. 


\subsection{2 ${ }^{1} \mathrm{H}-\mathrm{NMR}$ spectral data of binding experiments:}

3,4-Dimethoxyaniline (2a) and $\mathrm{Fe}[\mathrm{TPP}] \mathrm{Cl}$ in $\mathrm{CDCl}_{3}(400 \mathrm{MHz})($ Method A)

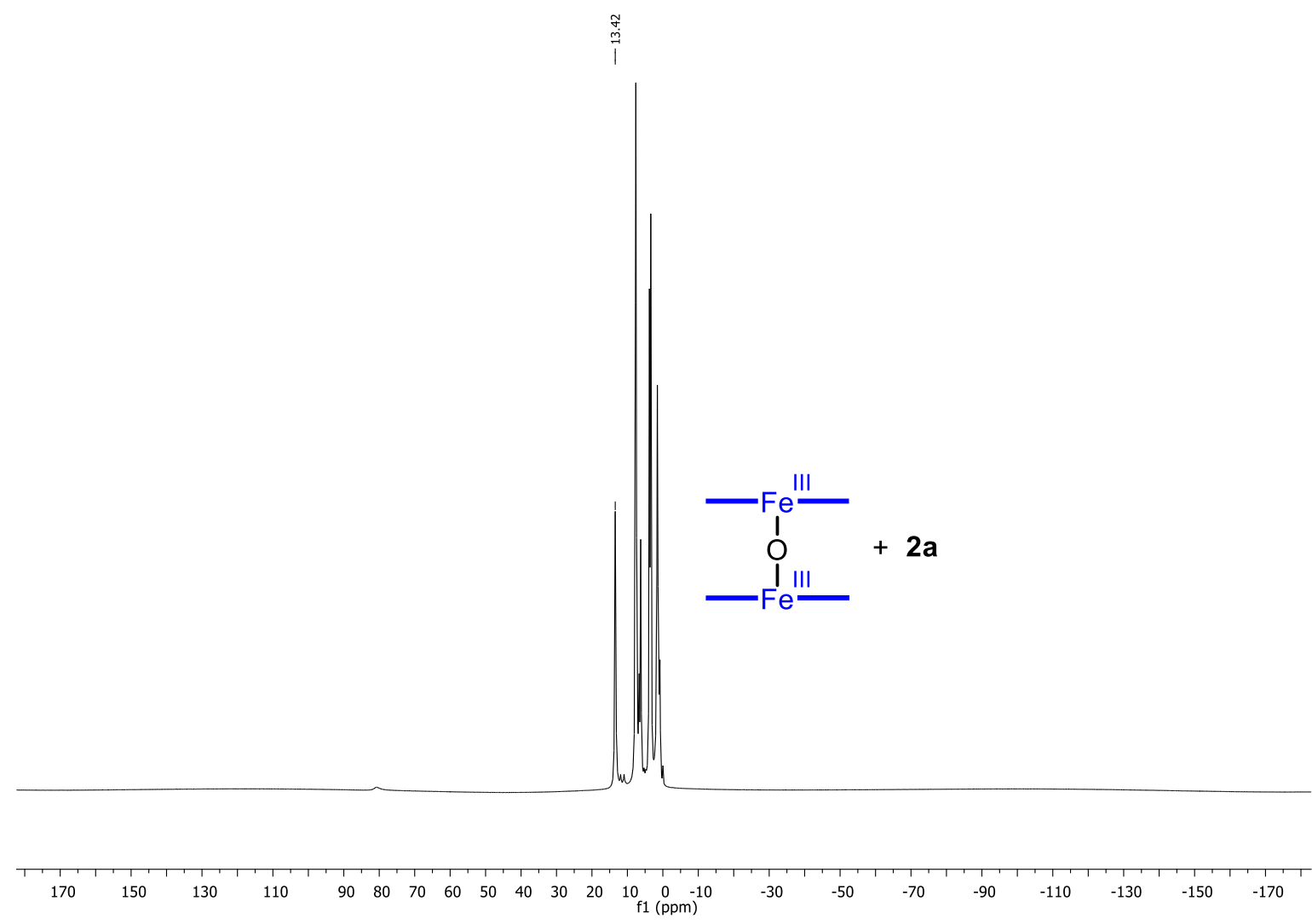

3,4-Dimethoxyaniline (2a), Fe[TPP]Cl and HFIP in $\mathrm{CDCl}_{3}(400 \mathrm{MHz})$ (Method B)

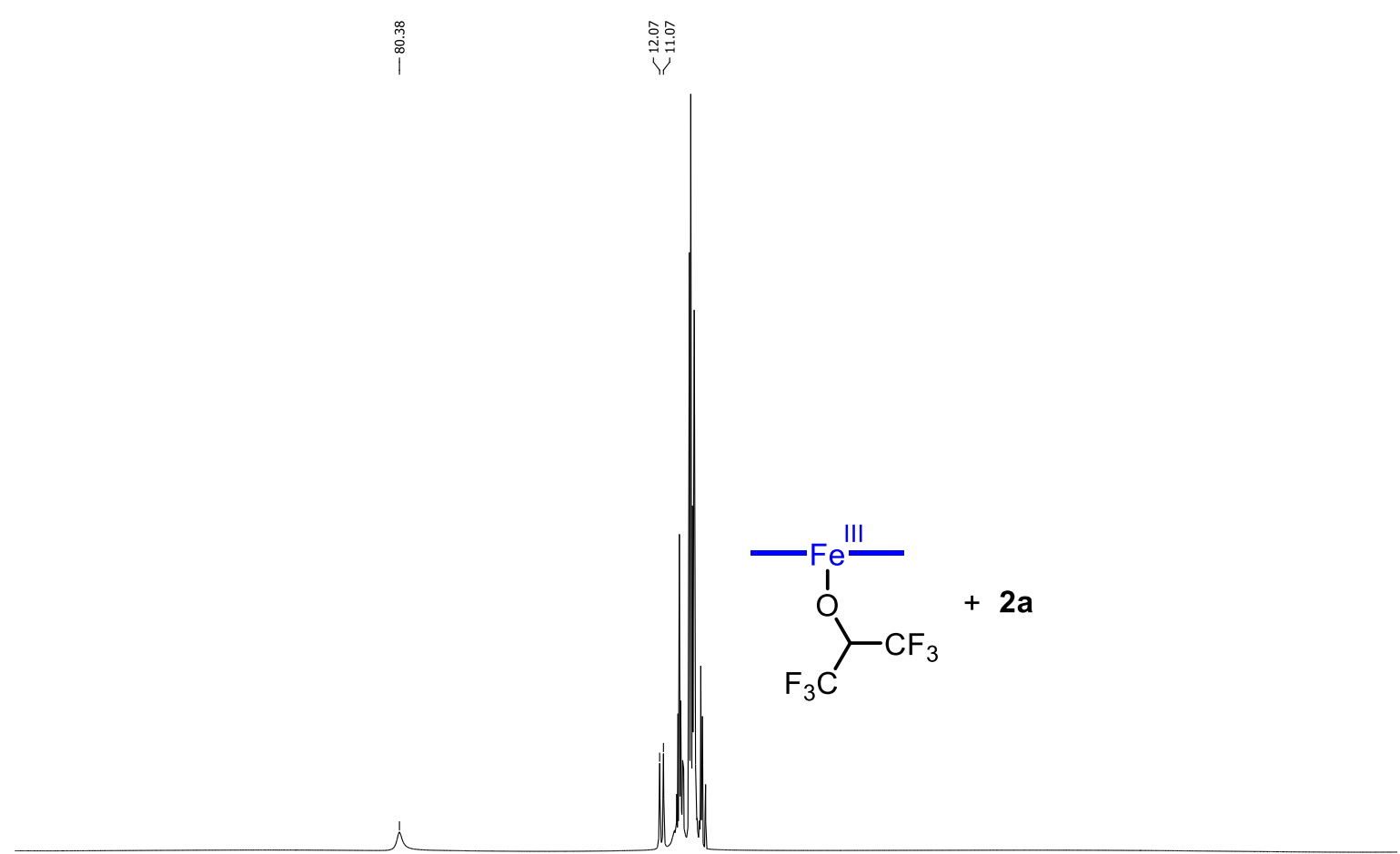

$\begin{array}{llllllllllllllllllllllllllll}170 & 150 & 130 & 110 & 90 & 80 & 70 & 60 & 50 & 40 & 30 & 20 & 10 & 0 & -10 & -30 & -50 & -70 & -90 & -110 & -130 & -150 & -170\end{array}$ 
2,4-Dimethoxyaniline (2b) and $\mathrm{Fe}[\mathrm{TPP}] \mathrm{Cl}$ in $\mathrm{CDCl}_{3}(400 \mathrm{MHz}$ ) (Method A)

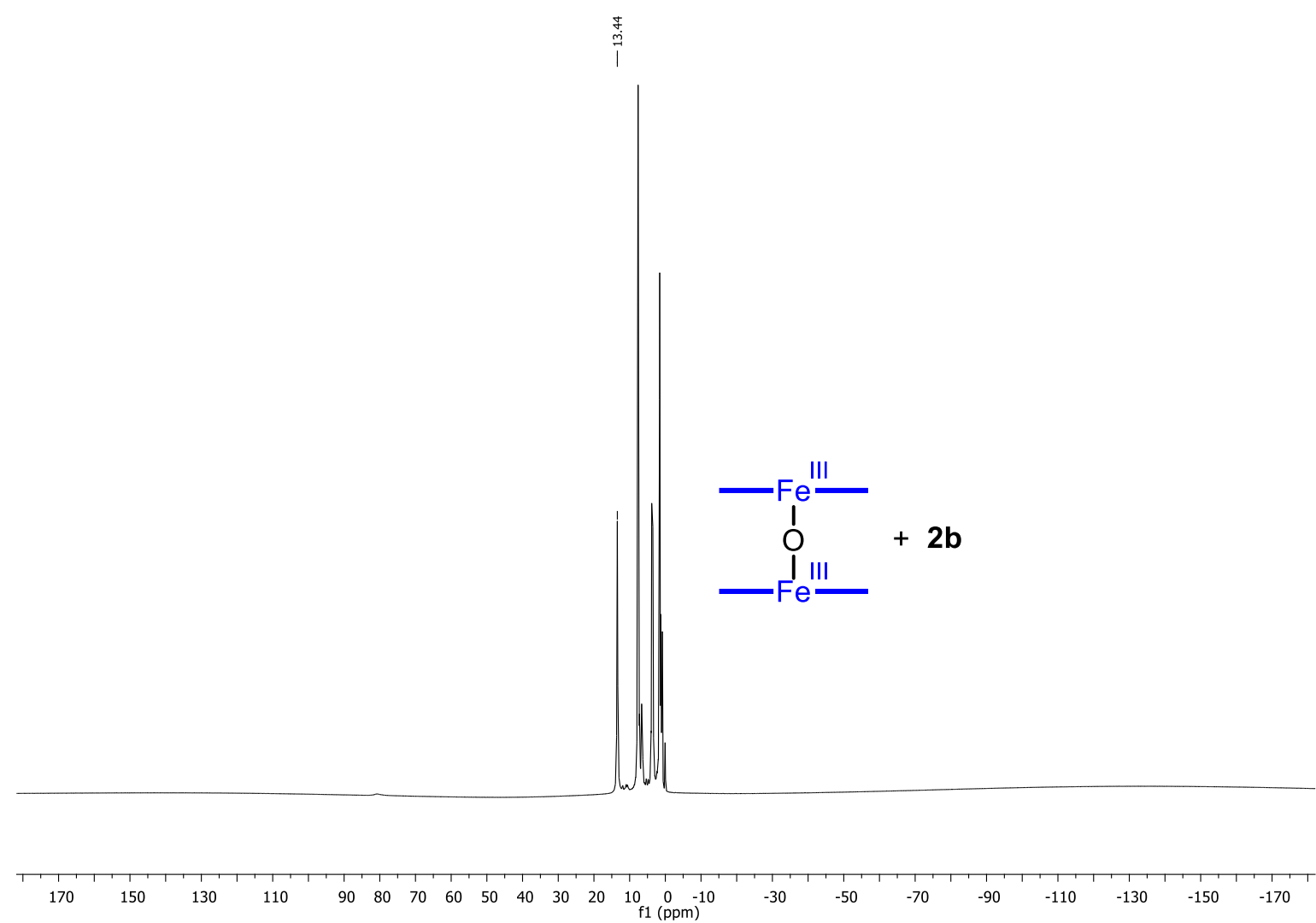

2,4-Dimethoxyaniline (2b), Fe[TPP]Cl and HFIP in $\mathrm{CDCl}_{3}(400 \mathrm{MHz})($ Method B)

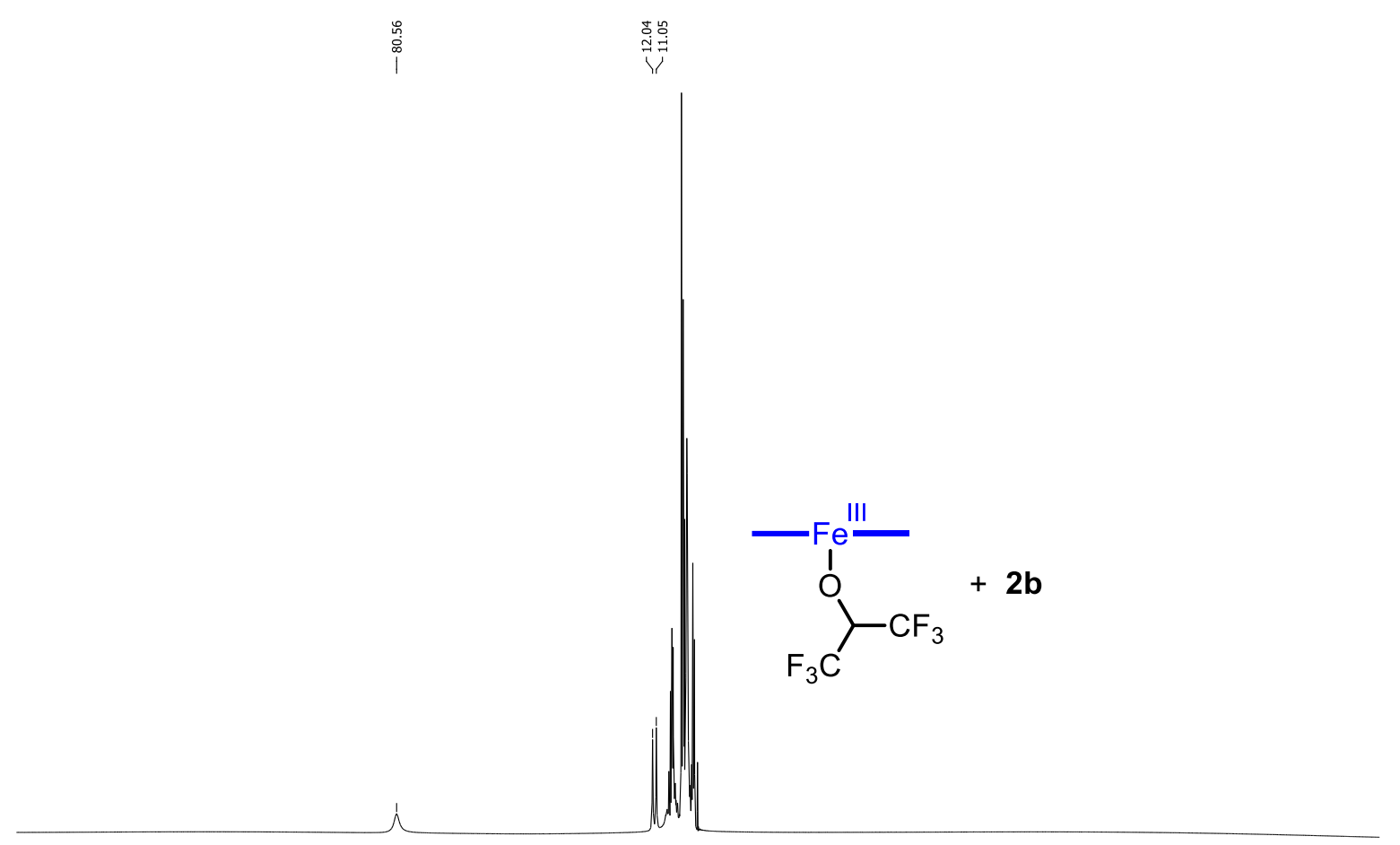


4,4'-dimethoxydiphenylamine (2d) and $\mathrm{Fe}[\mathrm{TPP}] \mathrm{Cl}$ in $\mathrm{CDCl}_{3}(400 \mathrm{MHz})$ (Method A)

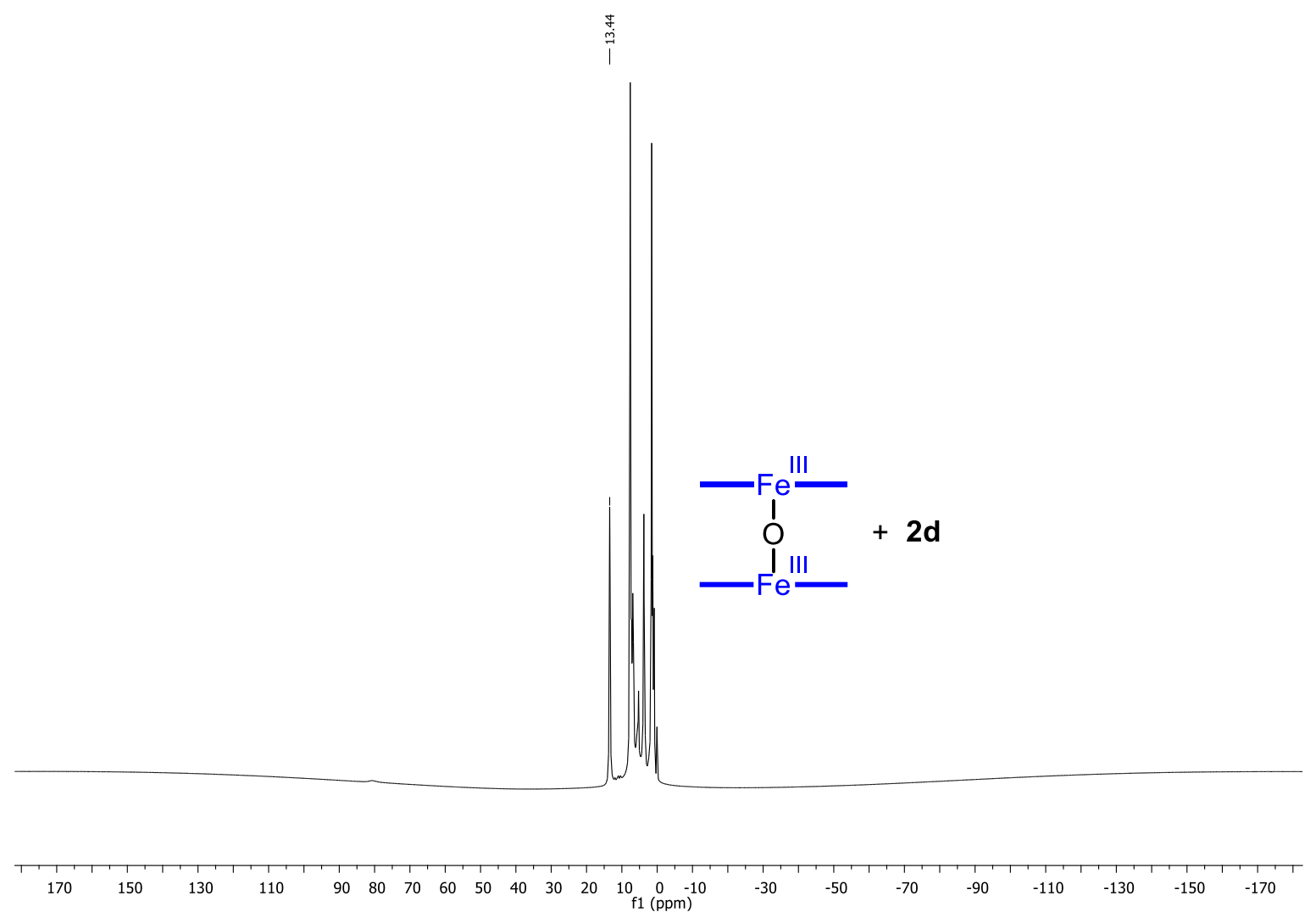

4,4'-dimethoxydiphenylamine (2d), Fe[TPP]Cl and HFIP in $\mathrm{CDCl}_{3}(400 \mathrm{MHz}$ ) (Method B)

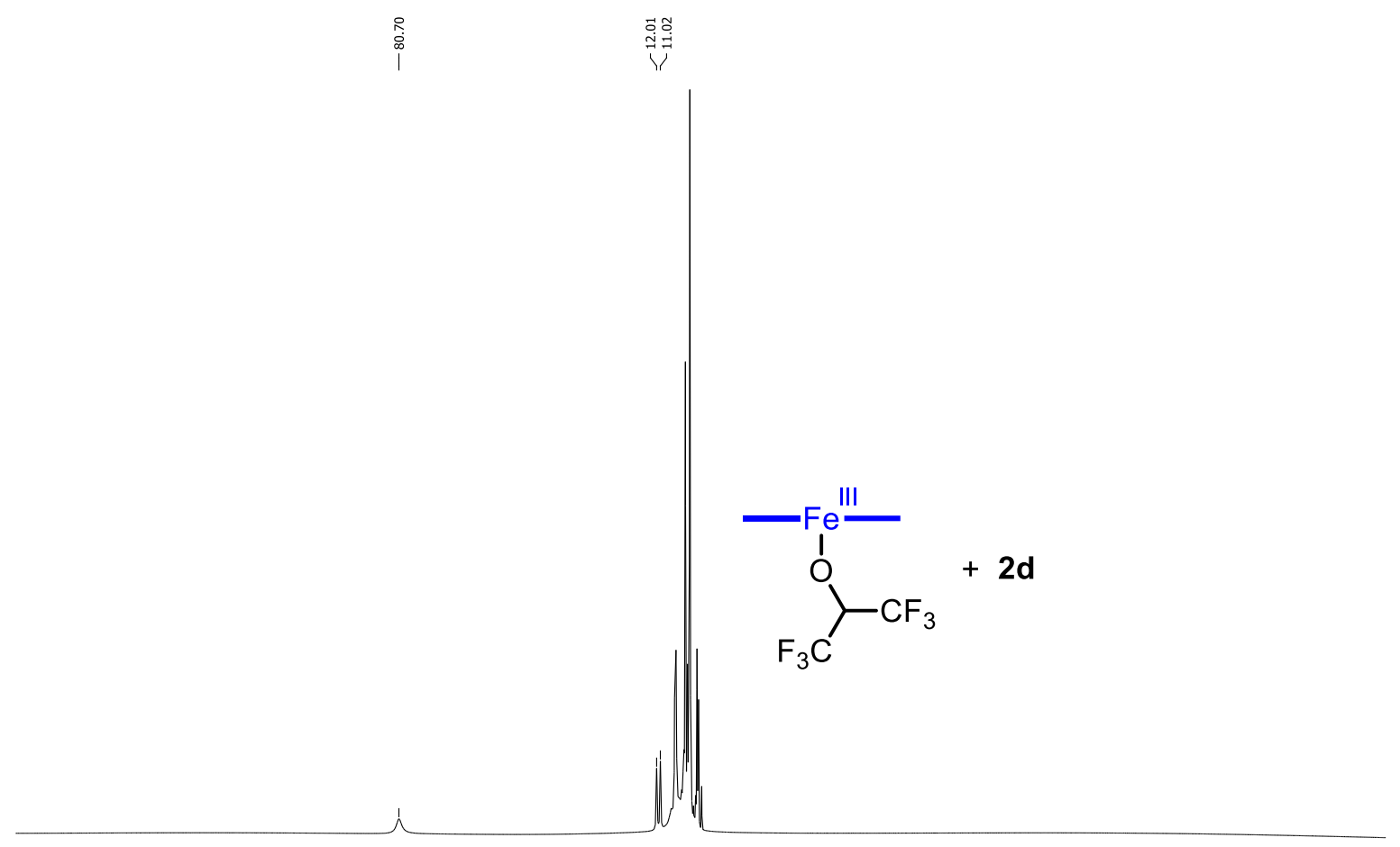


4-Methoxyaniline (2e) and $\mathrm{Fe}[\mathrm{TPP}] \mathrm{Cl}$ in $\mathrm{CDCl}_{3}(400 \mathrm{MHz}$ ) (Method A)
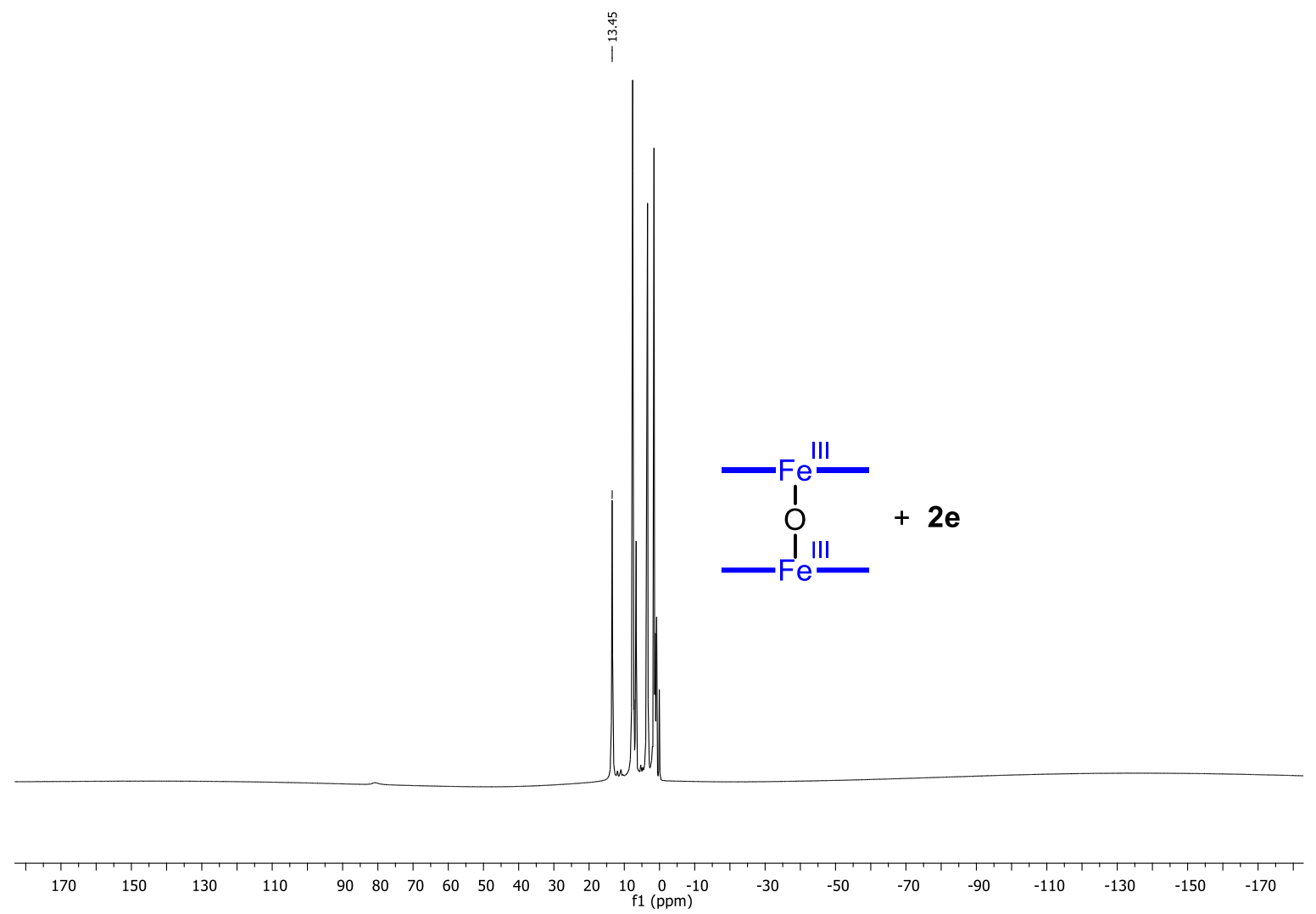

4-Methoxyaniline (2e), Fe[TPP]Cl and HFIP in $\mathrm{CDCl}_{3}(400 \mathrm{MHz})($ Method B)

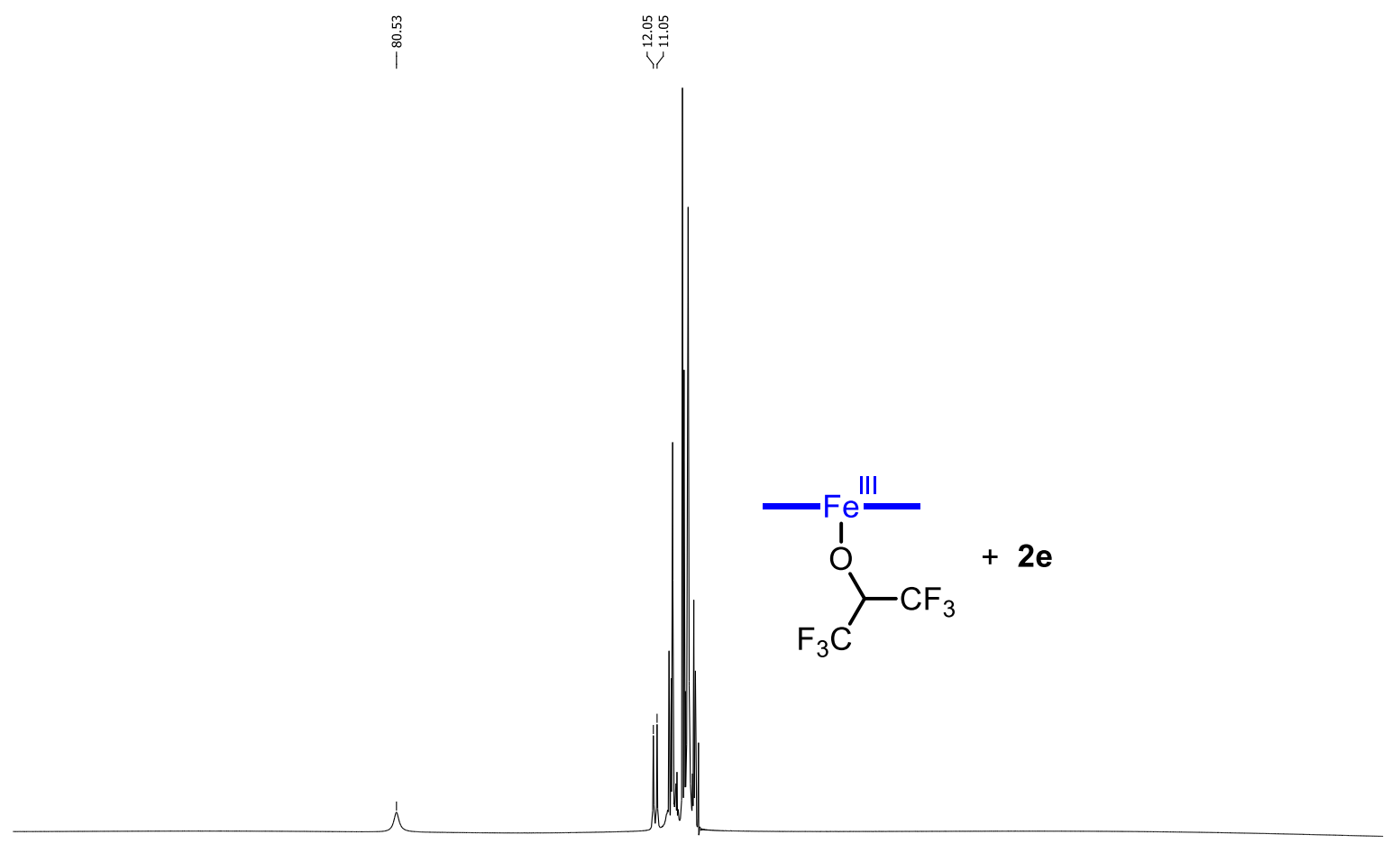


3-Methyl-4-methoxyaniline (2f) and $\mathrm{Fe}[\mathrm{TPP}] \mathrm{Cl}$ in $\mathrm{CDCl}_{3}(400 \mathrm{MHz}$ ) (Method A)

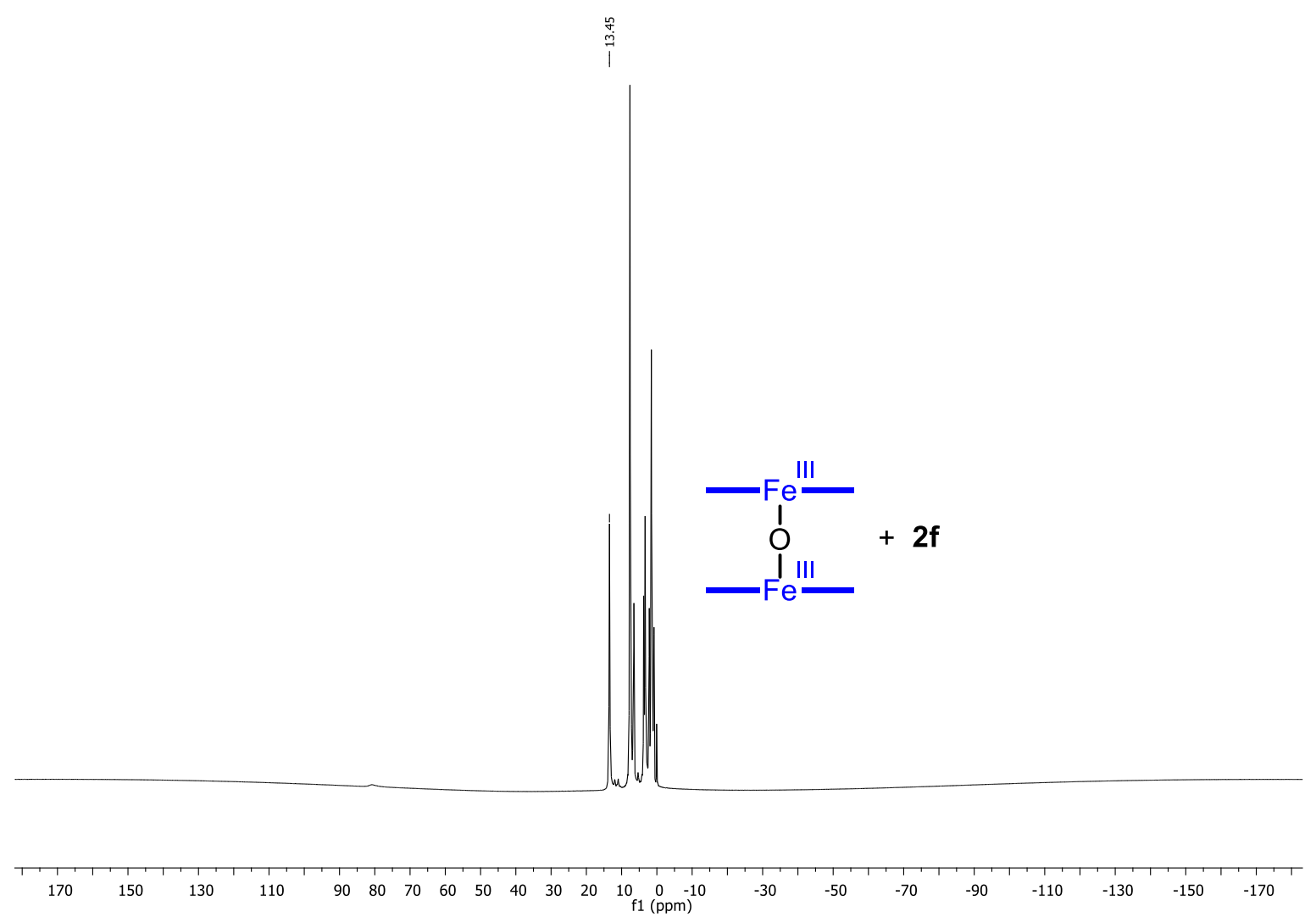

3-Methyl-4-methoxyaniline (2f), $\mathrm{Fe}[\mathrm{TPP}] \mathrm{Cl}$ and HFIP in $\mathrm{CDCl}_{3}(400 \mathrm{MHz}$ ) (Method B)

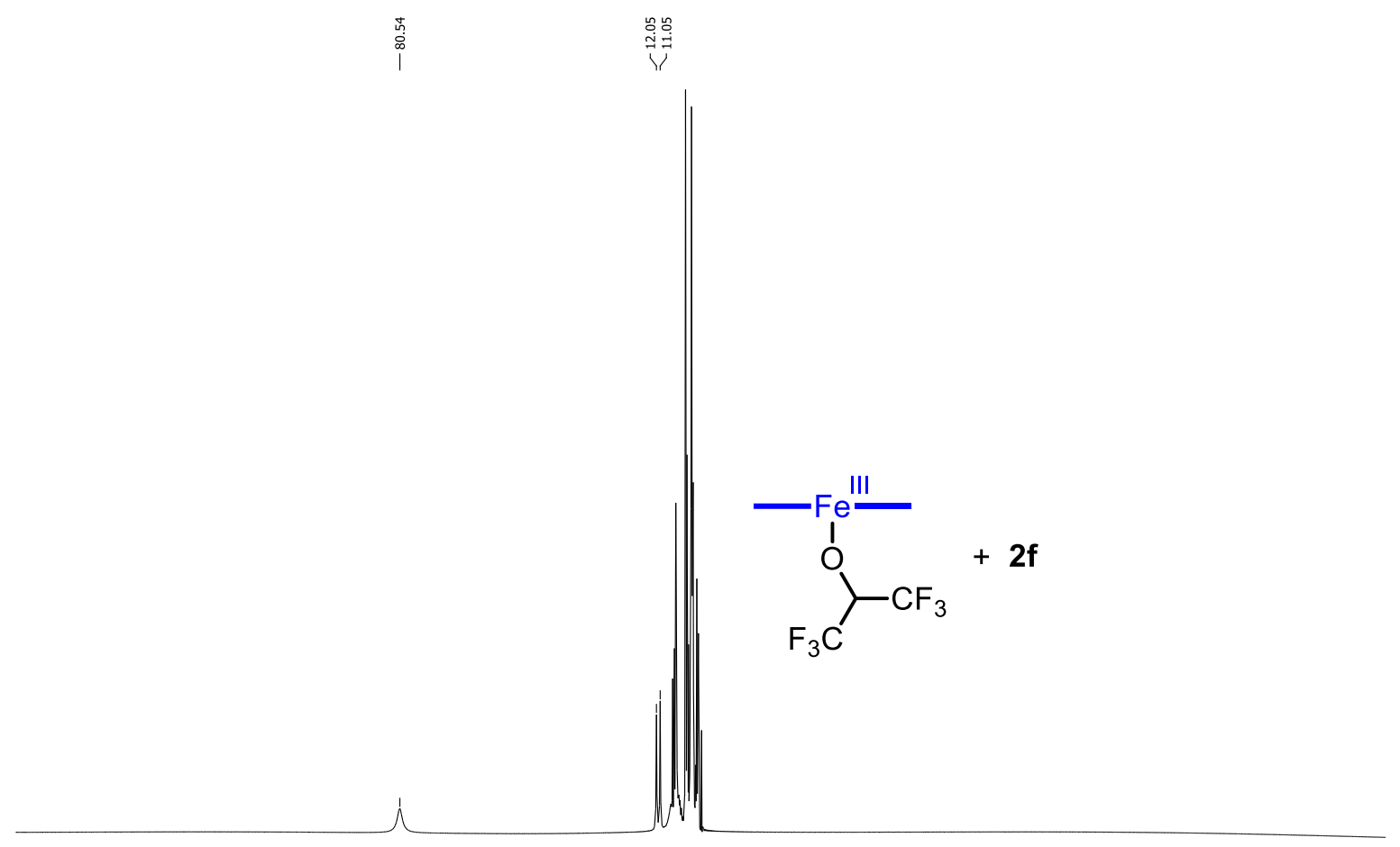


2-Methyl-4-methoxyaniline (2g) and $\mathrm{Fe}[\mathrm{TPP}] \mathrm{Cl}$ in $\mathrm{CDCl}_{3}(400 \mathrm{MHz}$ ) (Method A)

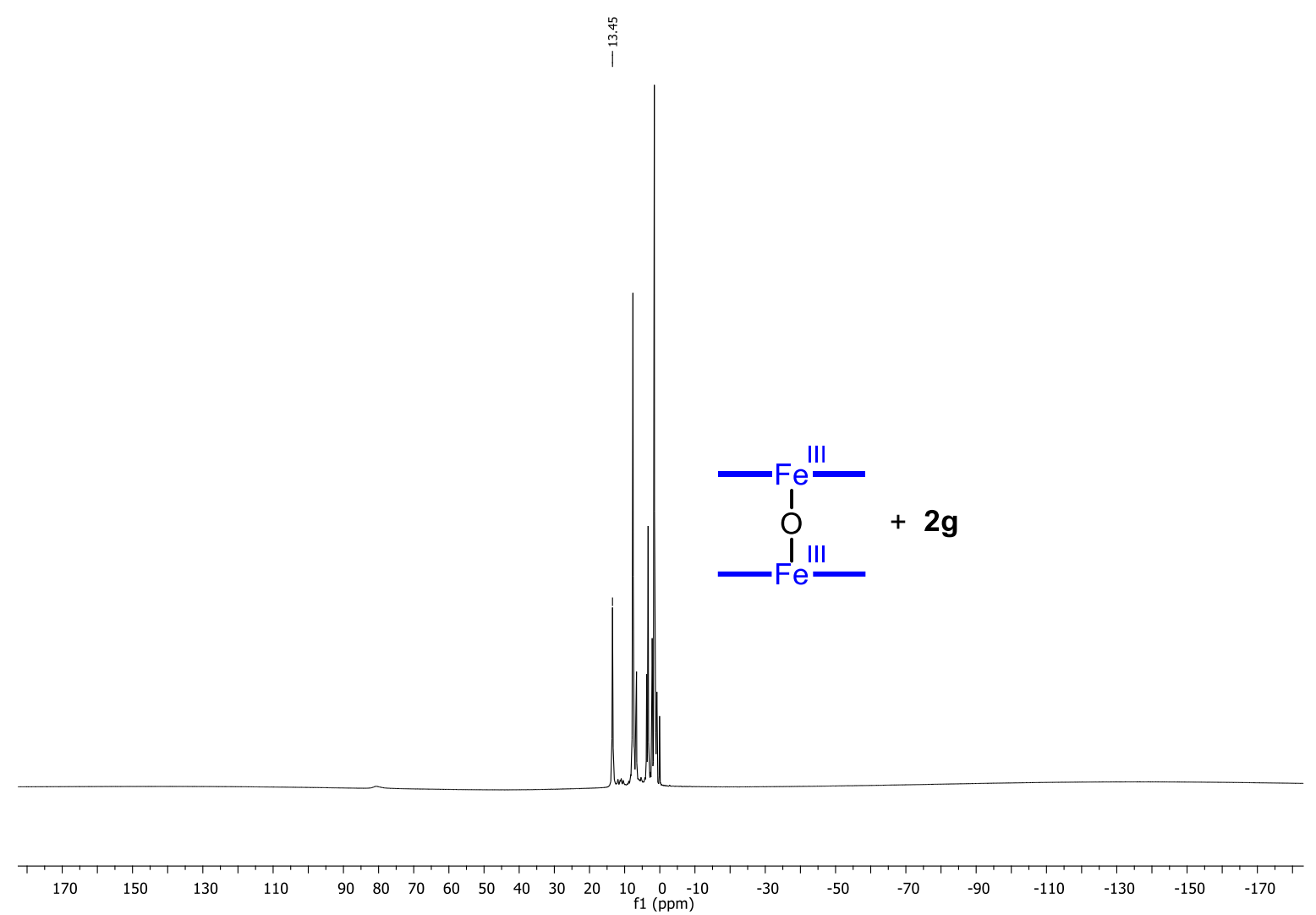

2-Methyl-4-methoxyaniline (2g), Fe[TPP]Cl and HFIP in $\mathrm{CDCl}_{3}(400 \mathrm{MHz})(\mathrm{Method} \mathrm{B})$

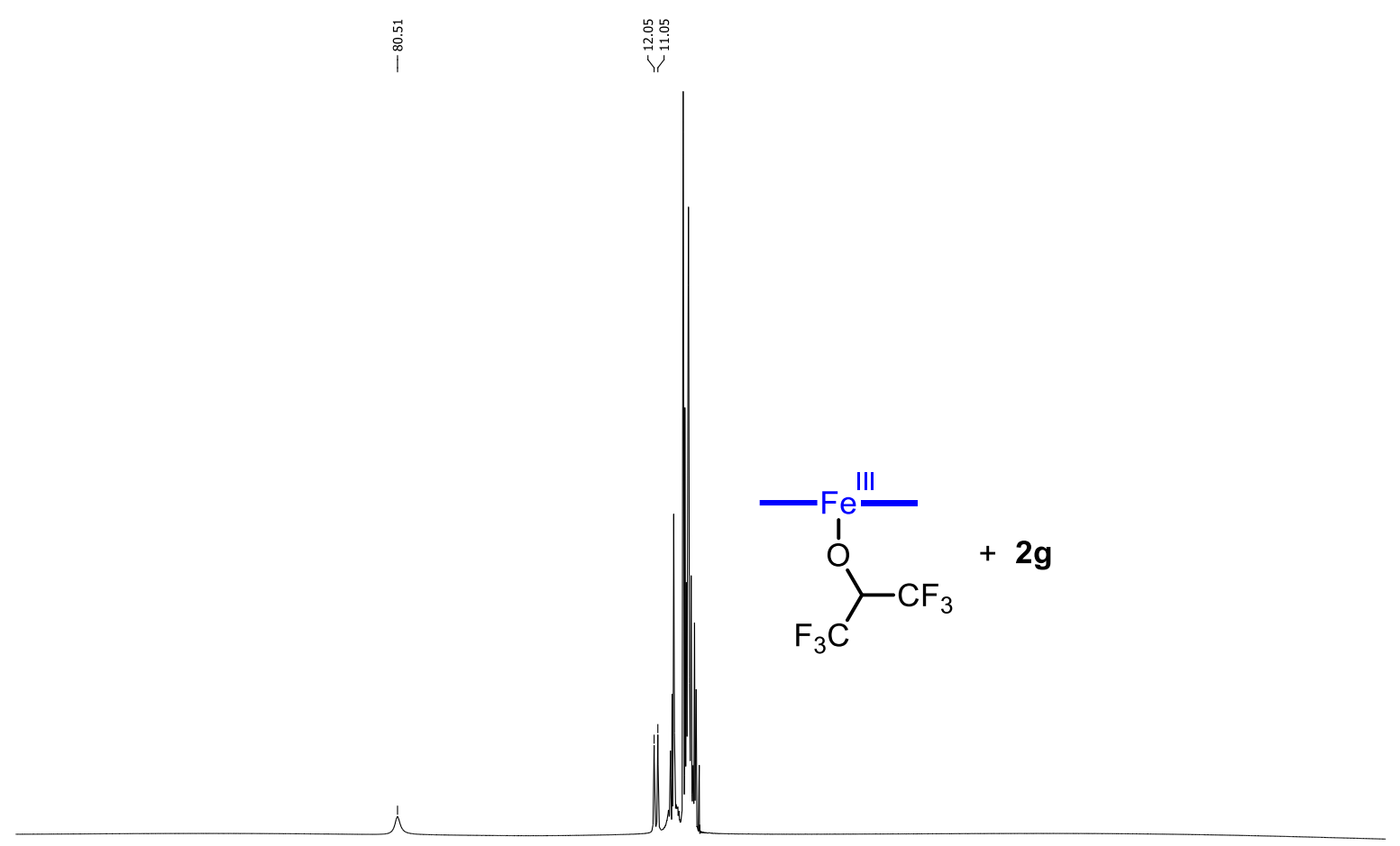

$\begin{array}{lllllllllllllllllllllllllllllll}170 & 150 & 130 & 110 & 90 & 80 & 70 & 60 & 50 & 40 & 30 & 20 & 10 & 0 & -10 & -30 & -50 & -70 & -90 & -110 & -130 & -150 & -170\end{array}$ 
2,6-Dimethylphenol (1b), 3,4-Dimethoxyaniline (2a) and $\mathrm{Fe}[\mathrm{TPP}] \mathrm{Cl}$ in $\mathrm{CDCl}_{3}(400 \mathrm{MHz})$ (Method C)

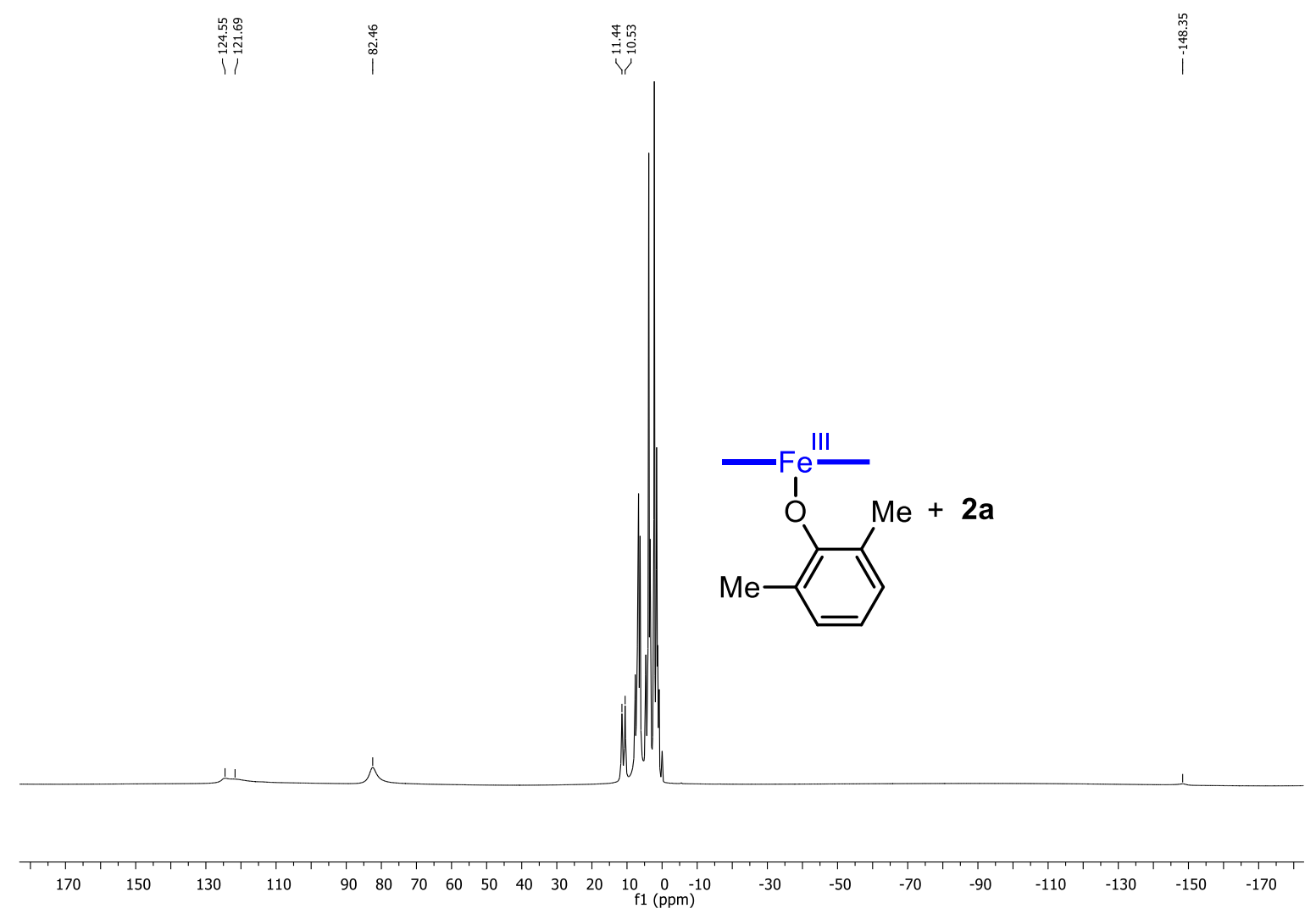

2,6-Dimethylphenol (1b), 3,4-Dimethoxyaniline (2a), $\mathrm{Fe}[\mathrm{TPP}] \mathrm{Cl}$ and $\mathrm{HFIP}$ in $\mathrm{CDCl}_{3}(400 \mathrm{MHz})$ (Method D)

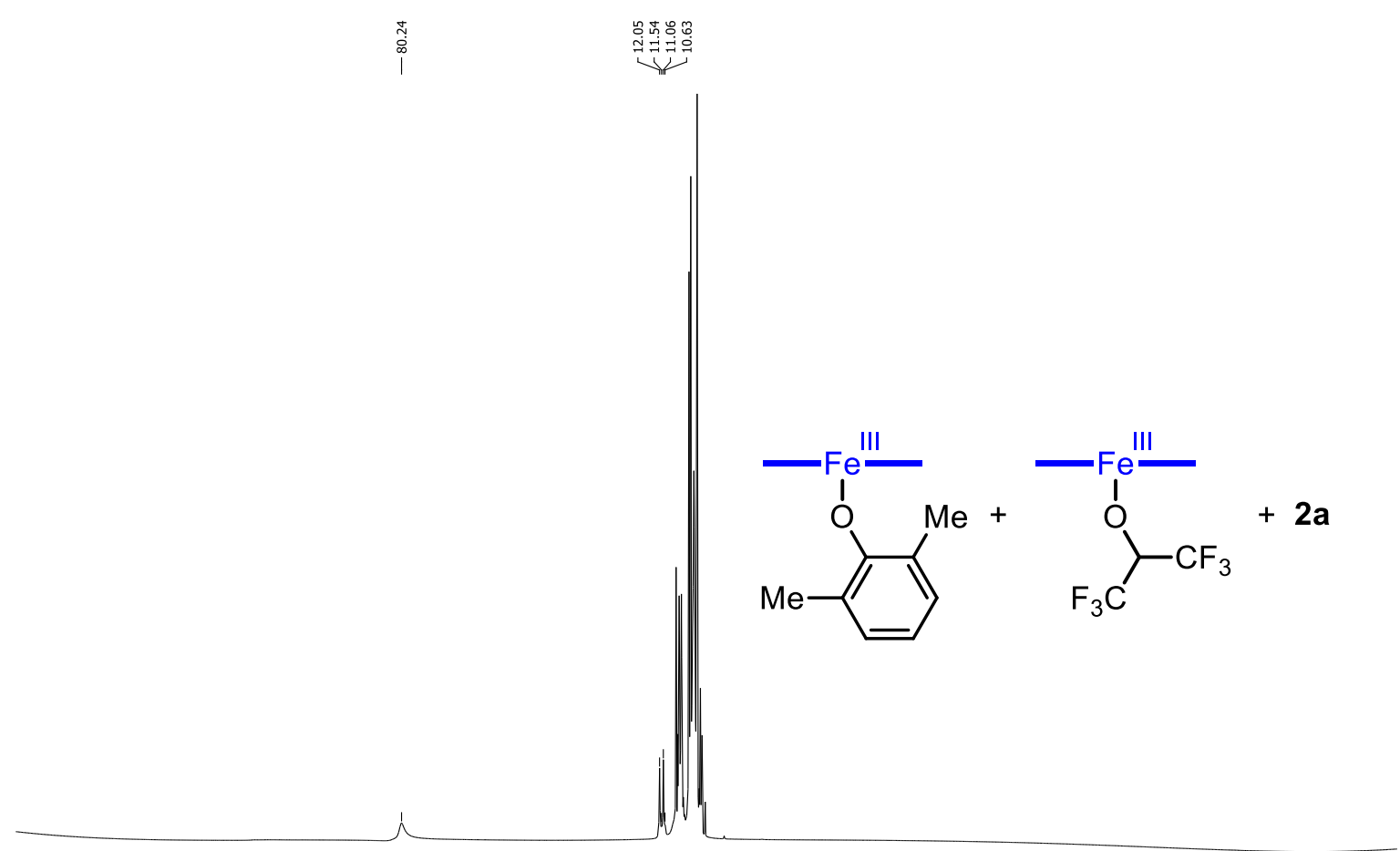


Discussion: The use of competitive binding experiments enables us to evaluate the binding strength of organic compounds to the Fe[TPP $] \mathrm{Cl} .{ }^{1}$ This set of experiments reveal that none of the anilines that were tested bind to the $\mathrm{Fe}[\mathrm{TPP}] \mathrm{Cl}$ complex in $\mathrm{CDCl}_{3}$, instead an $\mu$-oxo iron dimer complex $\left[(\mathrm{Fe}[\mathrm{TPP}])_{2} \mathrm{O}\right]$ is formed. In the presence of HFIP, the latter complex interacts with the solvent to afford $\mathrm{Fe}[\mathrm{TPP}]\left[\mathrm{OCH}\left(\mathrm{CF}_{3}\right)_{2}\right]$ complex. In addition, the competitive binding experiment between 2,6dimethylphenol (1b) and 3,4-dimethoxyaniline (2a) shows that $\mathbf{1 b}$ selectively binds to the $\mathrm{Fe}[\mathrm{TPP}] \mathrm{Cl}$ complex in $\mathrm{CDCl}_{3}$ forming $\mathrm{Fe}[\mathrm{TPP}][$ phenolate] complex. In the presence of HFIP the latter complex is found to be in an equilibrium with $\mathrm{Fe}[\mathrm{TPP}]\left[\mathrm{OCH}\left(\mathrm{CF}_{3}\right)_{2}\right]$ complex. In all of these experiments, binding of aniline to the iron was not observed, while phenols were found to be a strong axial ligands, even in the presence of HFIP.

\section{References:}

1. a) Reiss, H.; Shalit, H.; Vershinin, V.; More, N. Y.; Forckosh, H.; Pappo, D. Cobalt(II)[salen]Catalyzed Selective Aerobic Oxidative Cross-Coupling between Electron-Rich Phenols and 2Naphthols. J. Org. Chem. 2019, 84 (12), 7950-7960; b) Shalit, H.; Libman, A.; Pappo, D. mesoTetraphenylporphyrin Iron Chloride Catalyzed Selective Oxidative Cross-Coupling of Phenols. $J$. Am. Chem. Soc. 2017, 139 (38), 13404-13413. 


\section{Spectral $\left({ }^{1} \mathrm{H} \&{ }^{13} \mathrm{C}\right)$ data for compounds 3-38:}

${ }^{1} \mathrm{H}$ - NMR spectrum of compound $3\left(400 \mathrm{MHz}, \mathrm{CDCl}_{3}\right)$<smiles>COC1=CC(=Nc2ccc(OC)c(OC)c2)C=C(OC)C1=O</smiles>

Vுن

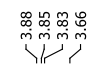
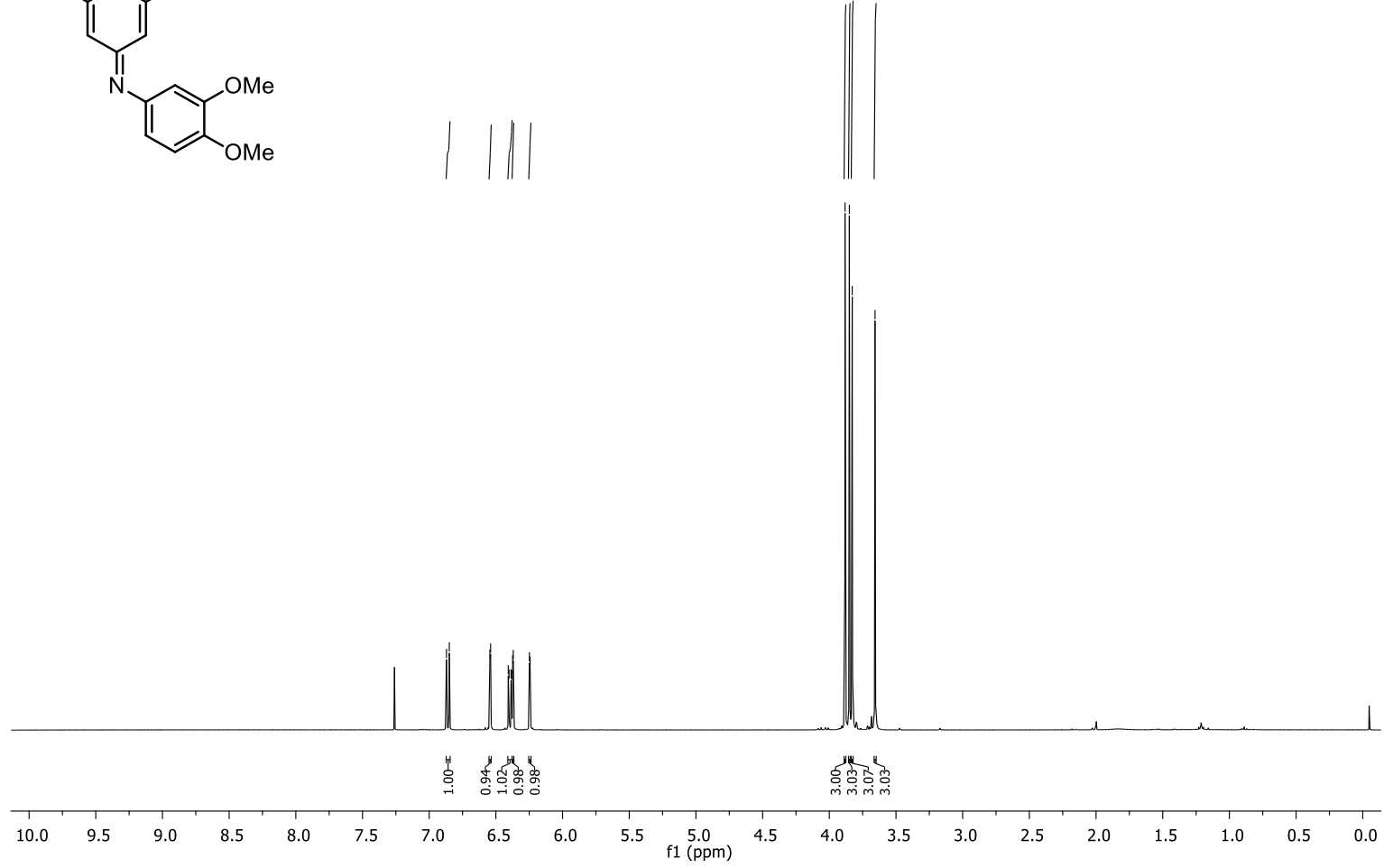

${ }^{13} \mathrm{C}$ - NMR spectrum of compound $3\left(100 \mathrm{MHz}, \mathrm{CDCl}_{3}\right)$

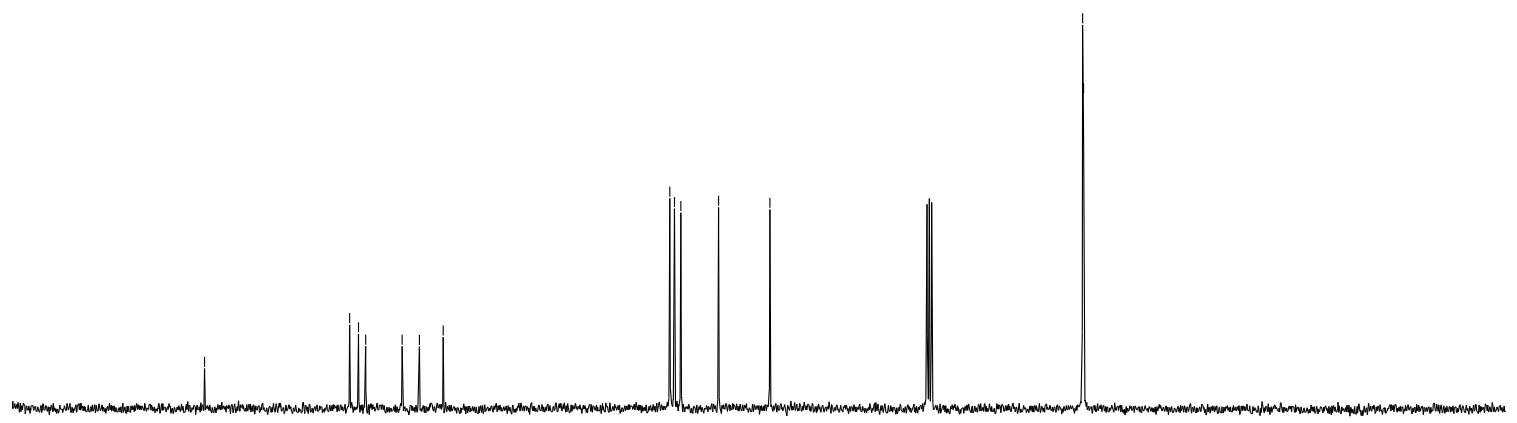

200

$\begin{array}{lllllllll}180 & 170 & 160 & 150 & 140 & 130 & 120 & 110 & \begin{array}{c}100 \\ f 1(\mathrm{ppm})\end{array}\end{array}$

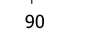

70

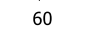


${ }^{1} \mathrm{H}$ - NMR spectrum of compound $4\left(400 \mathrm{MHz}, \mathrm{CDCl}_{3}\right)$

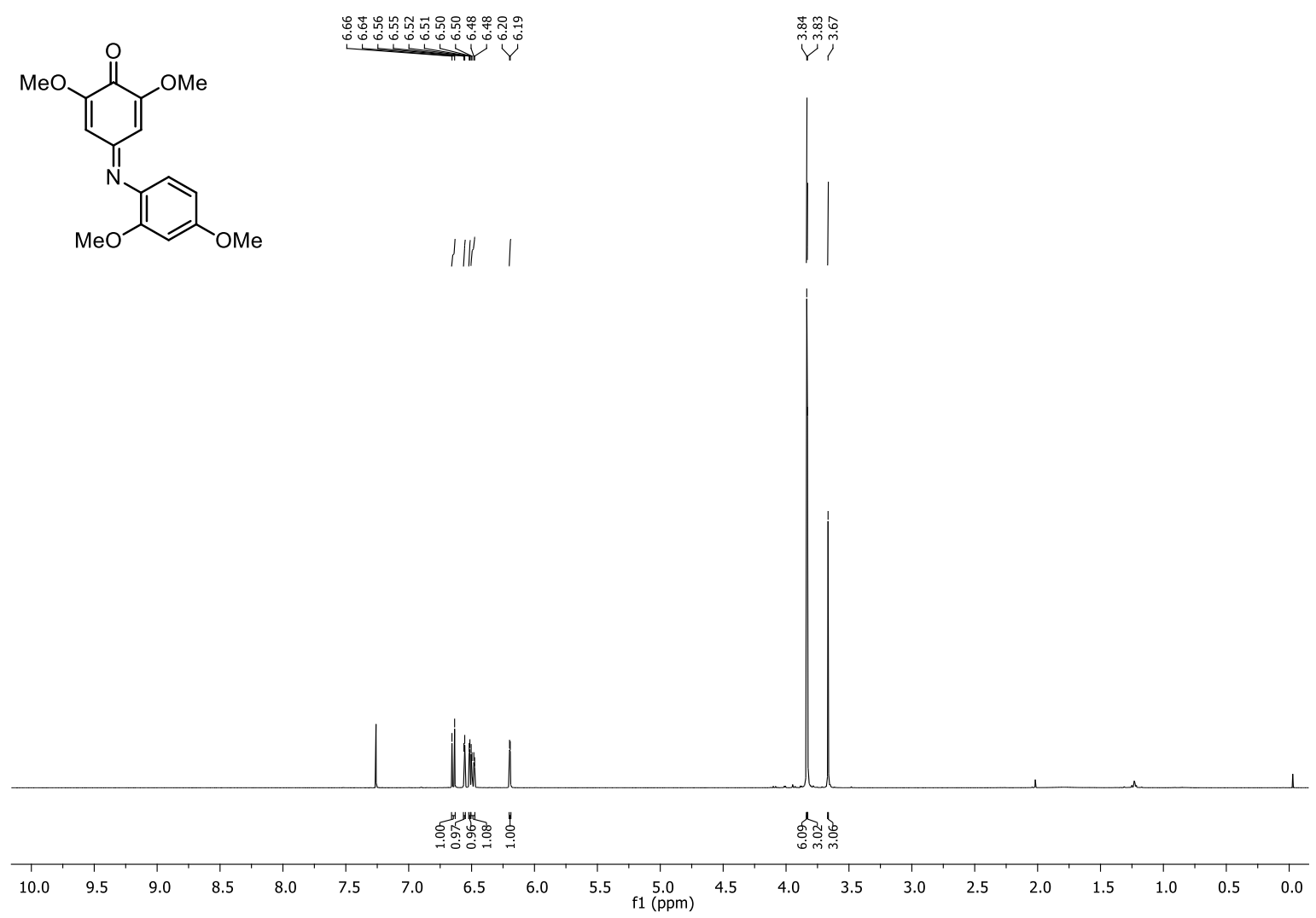

${ }^{13} \mathrm{C}-\mathrm{NMR}$ spectrum of compound $4\left(100 \mathrm{MHz}, \mathrm{CDCl}_{3}\right)$

|

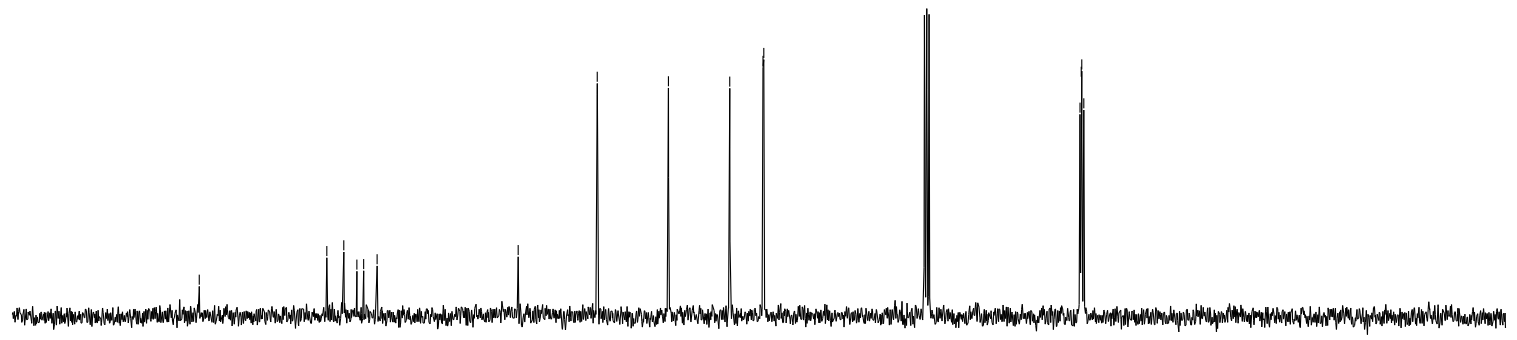

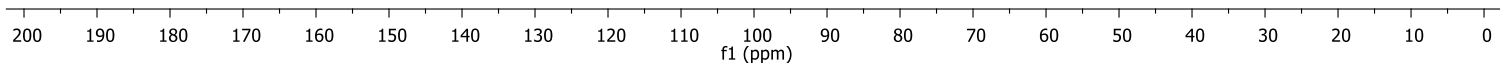


${ }^{1} \mathrm{H}$ - NMR spectrum of compound $5\left(400 \mathrm{MHz}, \mathrm{CDCl}_{3}\right.$ )
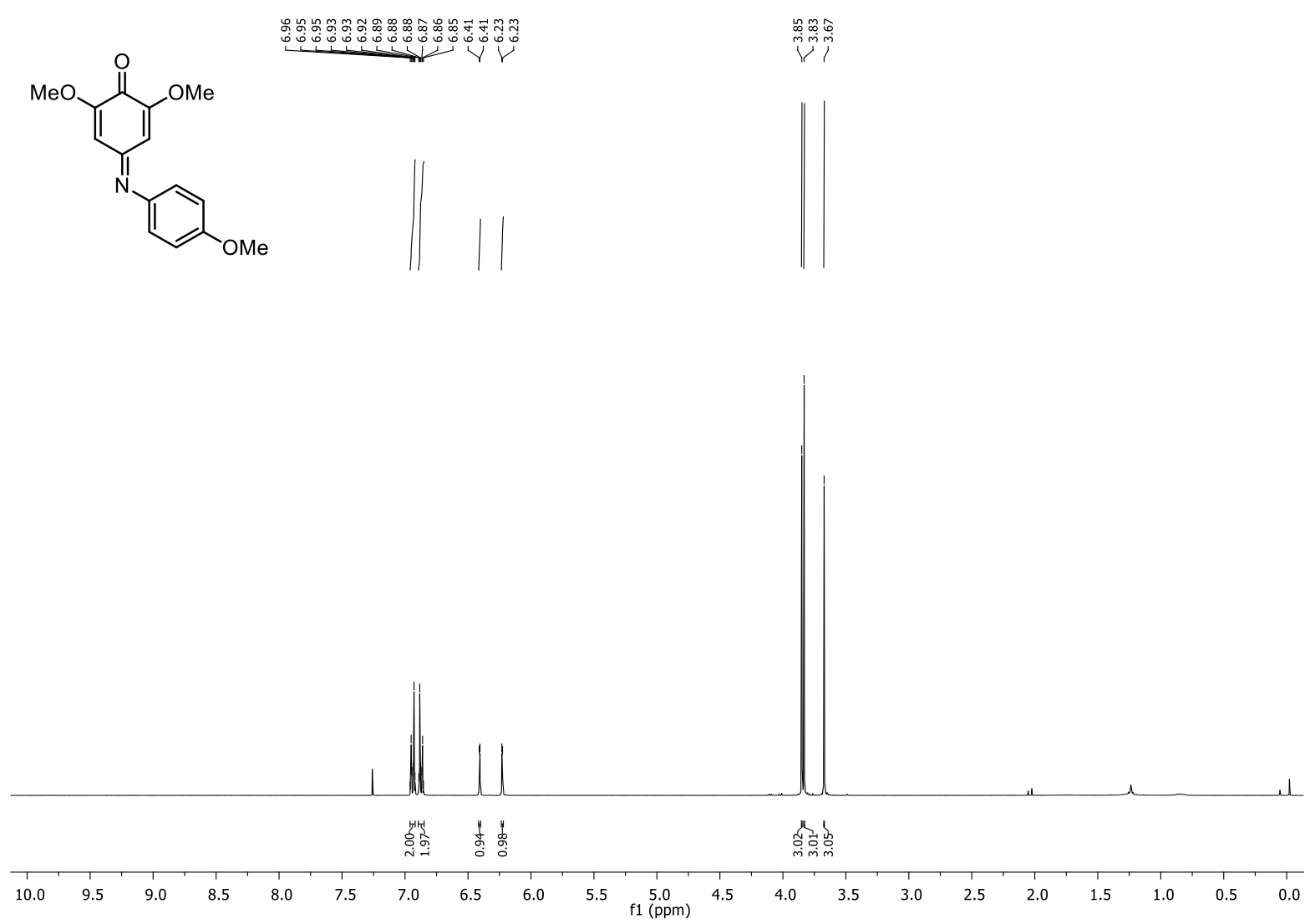

${ }^{13} \mathrm{C}$ - NMR spectrum of compound $5\left(100 \mathrm{MHz}, \mathrm{CDCl}_{3}\right)$

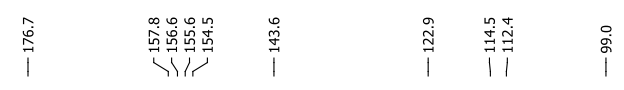
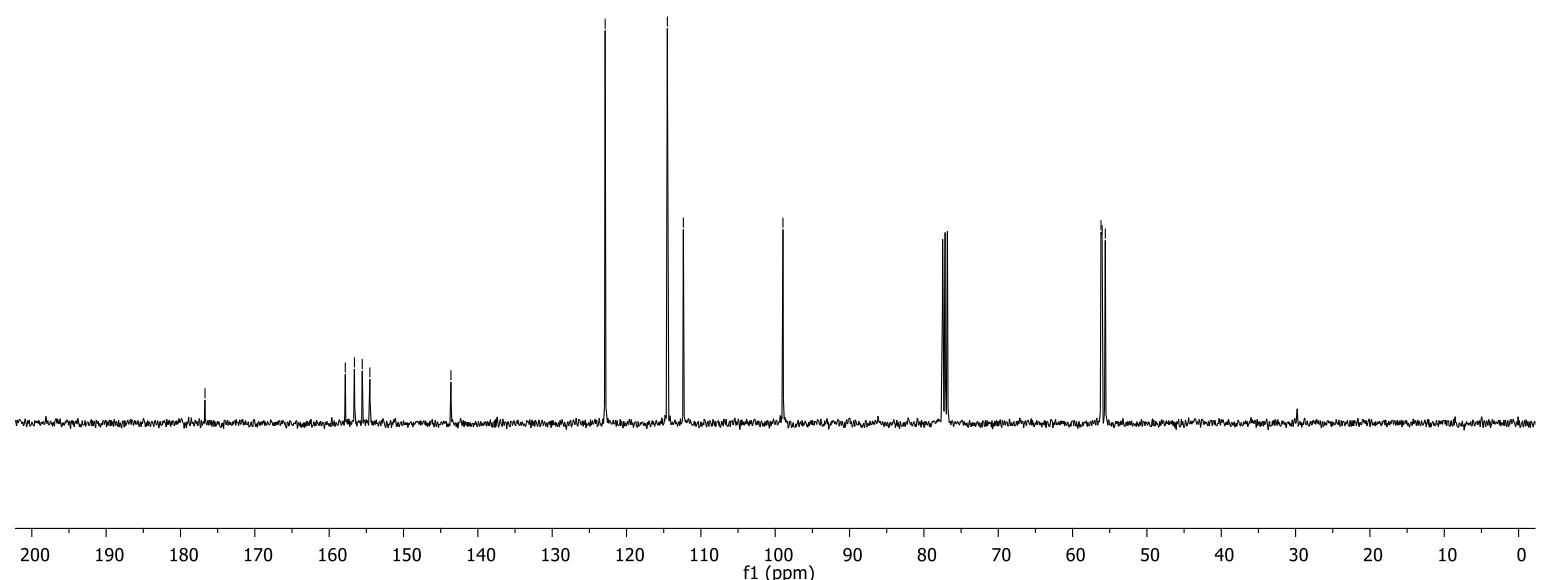
${ }^{1} \mathrm{H}$ - NMR spectrum of compound 6 (400 MHz, DMSO- $d_{6}$ )<smiles></smiles>
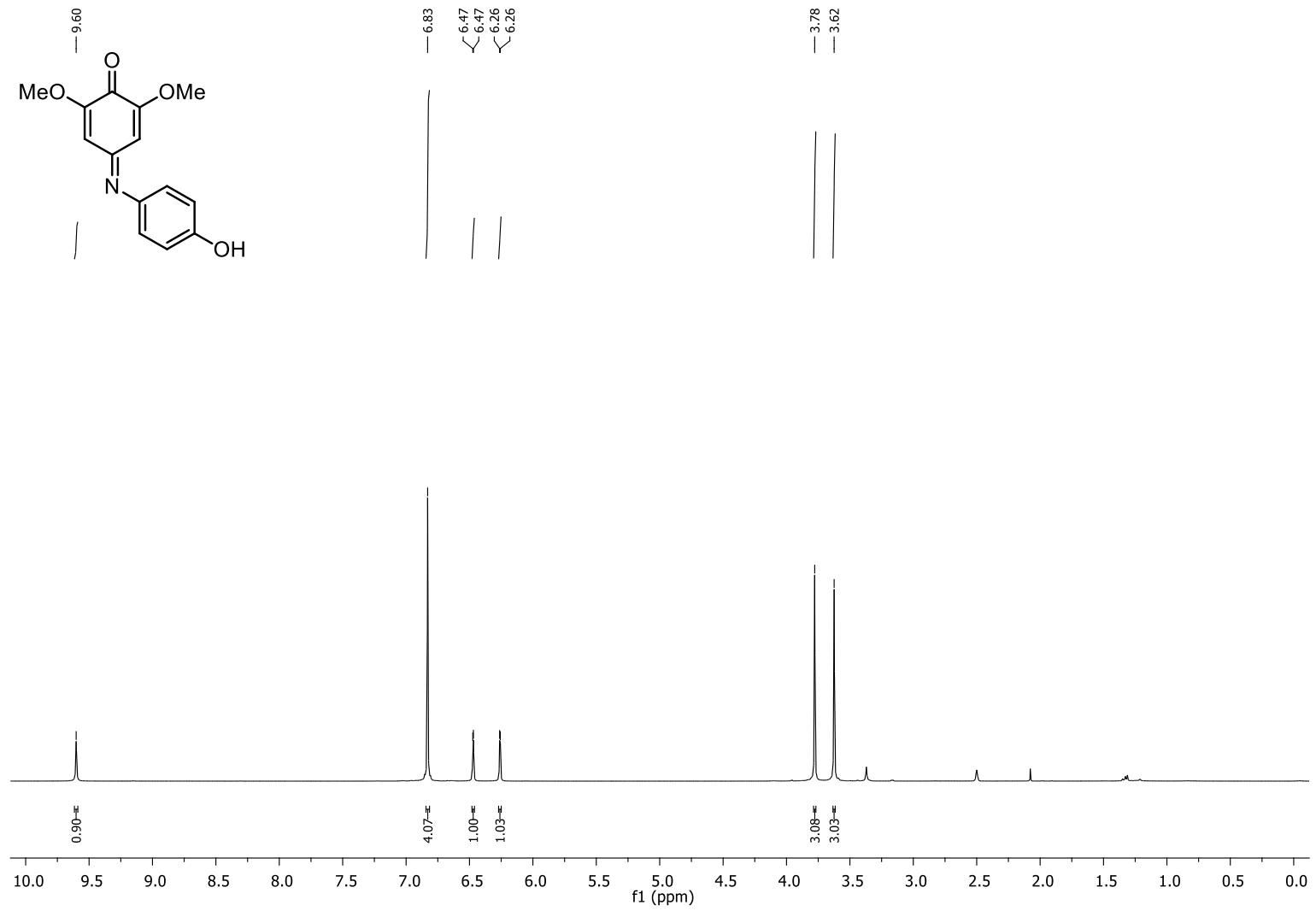

${ }^{13} \mathrm{C}$ - NMR spectrum of compound 6 (100 MHz, DMSO-d $\left.d_{6}\right)$

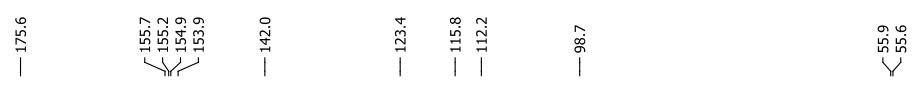

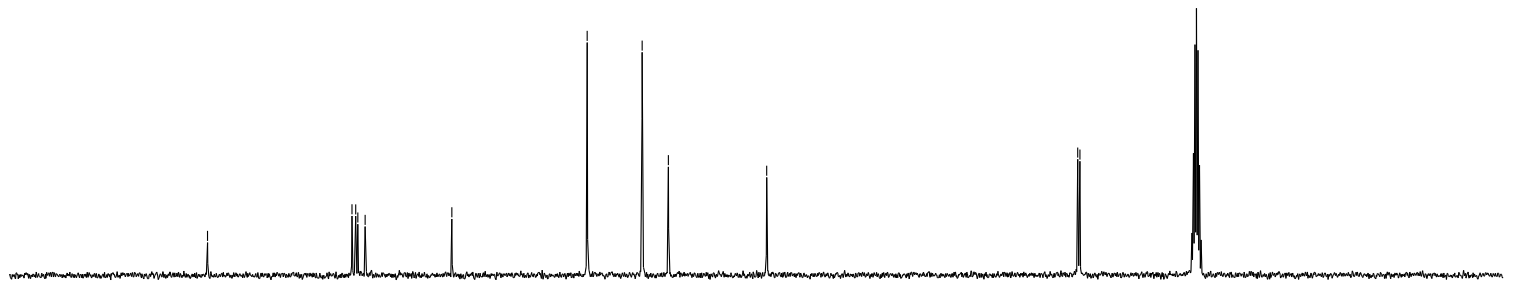

$\begin{array}{llllllllllllllllllllll}200 & 190 & 180 & 170 & 160 & 150 & 140 & 130 & 120 & 110 & 100 & 90 & 80 & 70 & 60 & 50 & 40 & 30 & 20 & 10 & 0\end{array}$ 
${ }^{1} \mathrm{H}$ - NMR spectrum of compound 7 (400 MHz, $\mathrm{CDCl}_{3}$ )
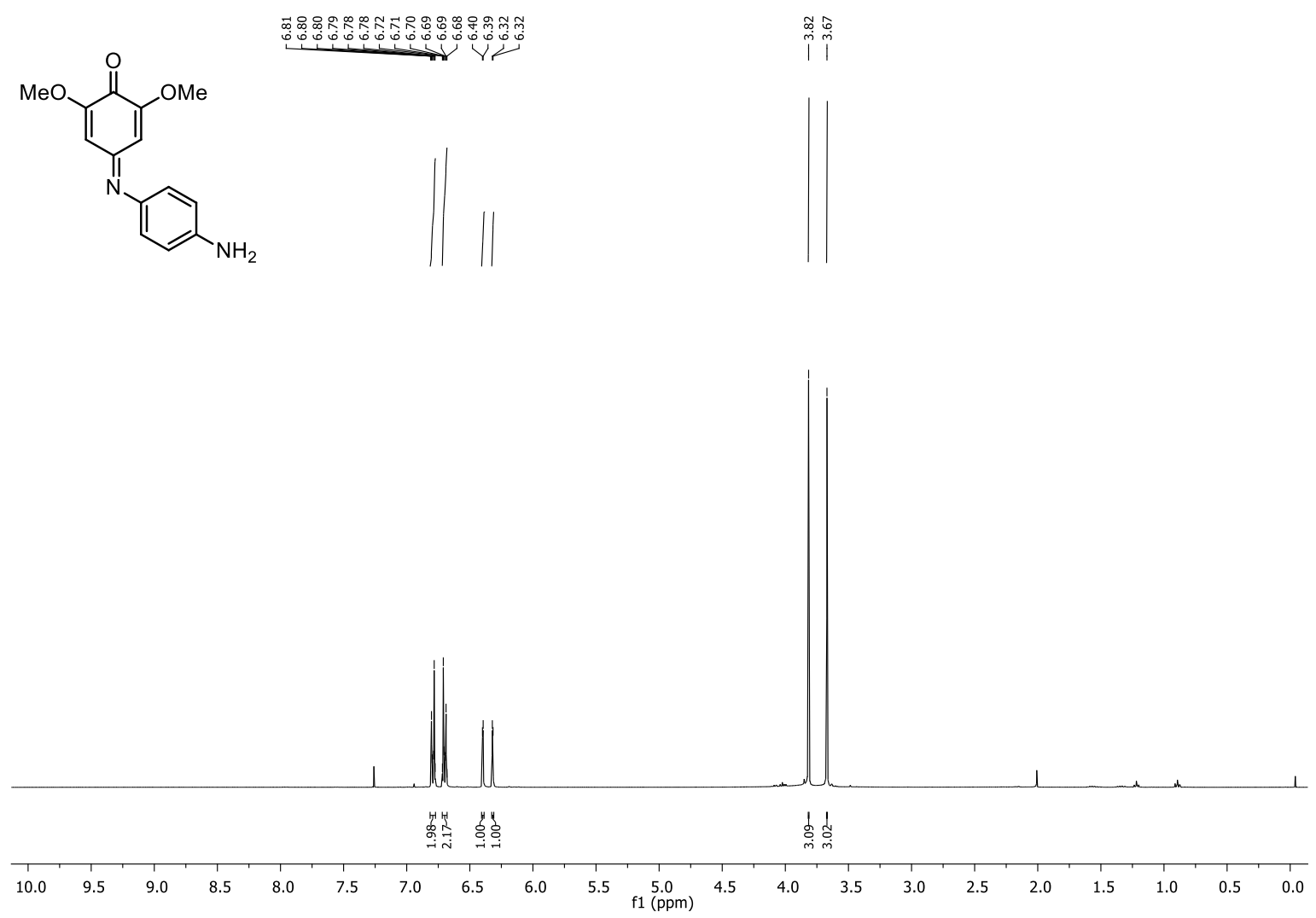

${ }^{13} \mathrm{C}$ - NMR spectrum of compound $7\left(100 \mathrm{MHz}, \mathrm{CDCl}_{3}\right)$

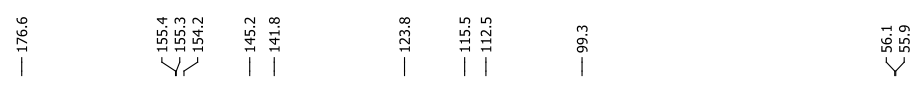

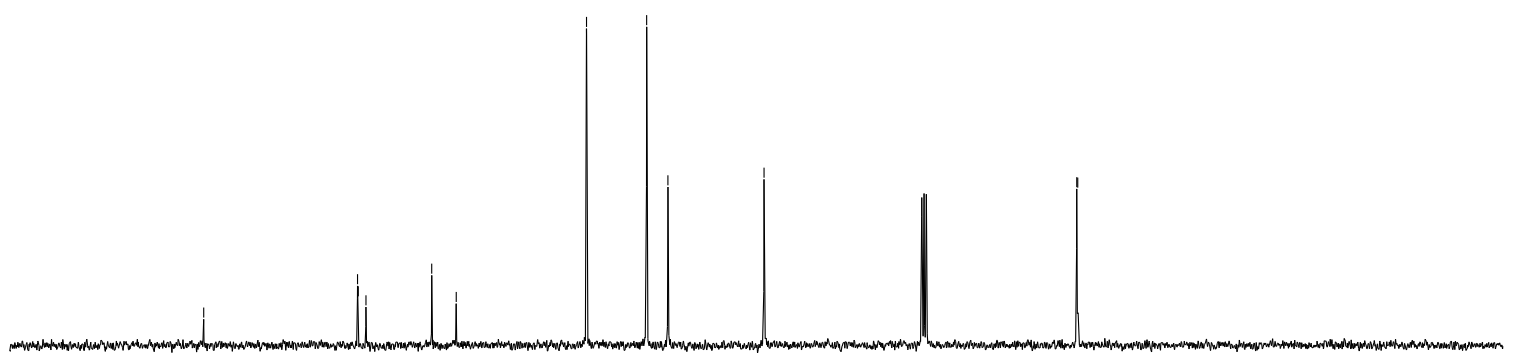


${ }^{1} \mathrm{H}-\mathrm{NMR}$ spectrum of compound $\mathbf{8}\left(400 \mathrm{MHz}, \mathrm{CDCl}_{3}\right)$

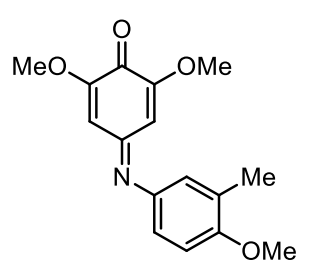

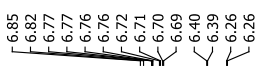

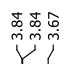

$\underset{\widetilde{*}}{\text { i }}$

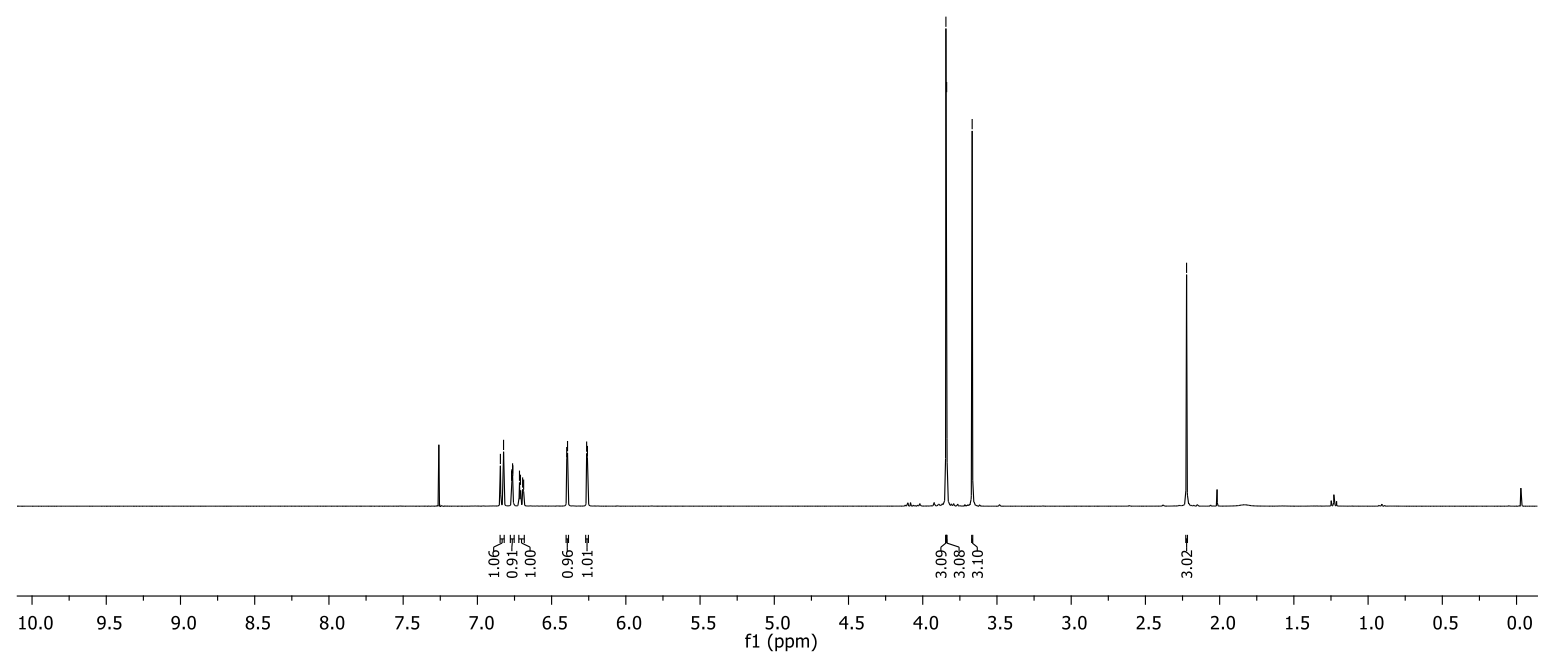

${ }^{13} \mathrm{C}$ - NMR spectrum of compound $8\left(100 \mathrm{MHz}, \mathrm{CDCl}_{3}\right)$

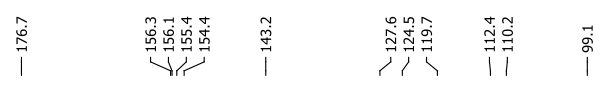

ไู่

$\stackrel{+}{\stackrel{0}{0}}$
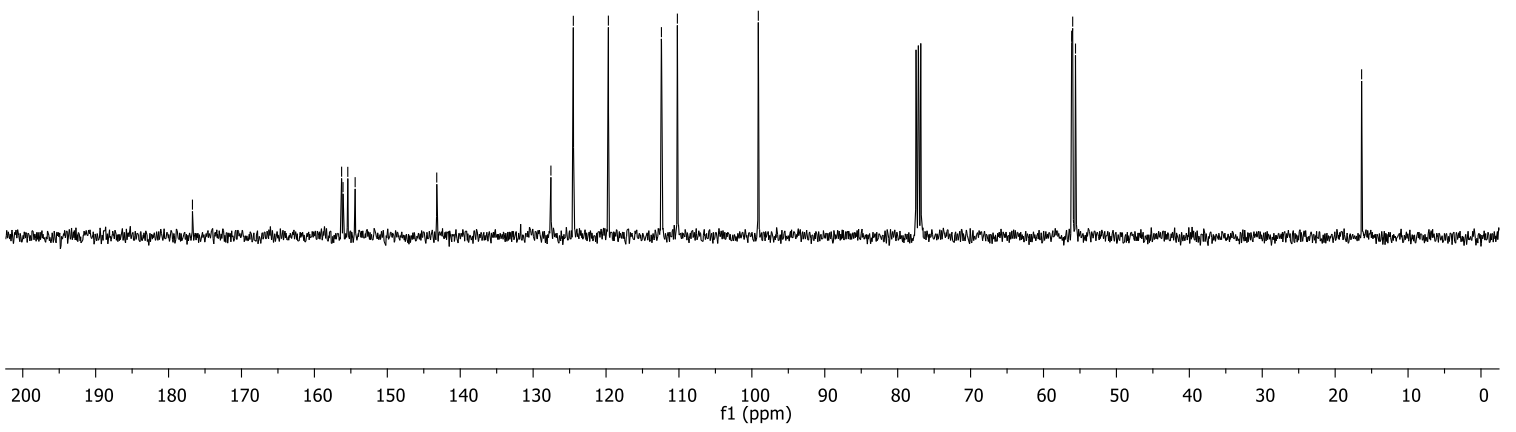

S32 
${ }^{1} \mathrm{H}$ - NMR spectrum of compound 9 (400 $\left.\mathrm{MHz}, \mathrm{CDCl}_{3}\right)$

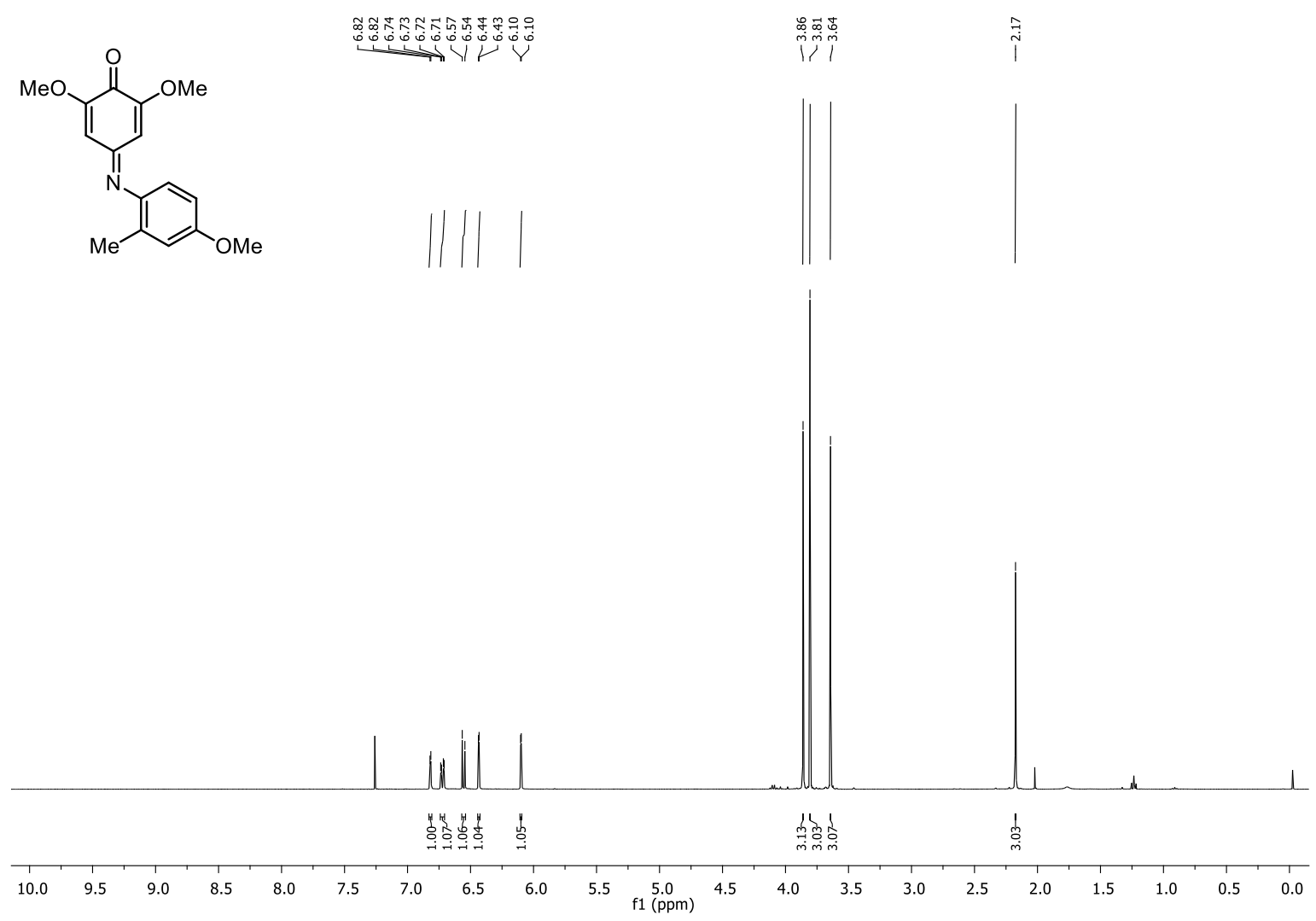

${ }^{13} \mathrm{C}$ - NMR spectrum of compound $9\left(100 \mathrm{MHz}, \mathrm{CDCl}_{3}\right)$

i

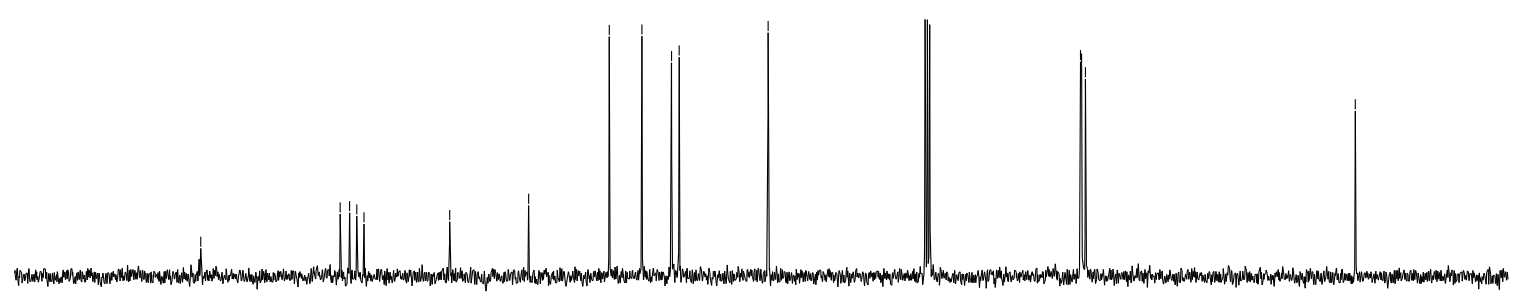

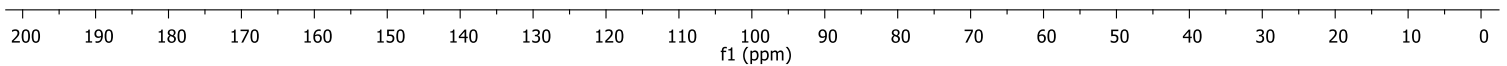


${ }^{1} \mathrm{H}$ - NMR spectrum of compound $10\left(400 \mathrm{MHz}, \mathrm{CDCl}_{3}\right)$

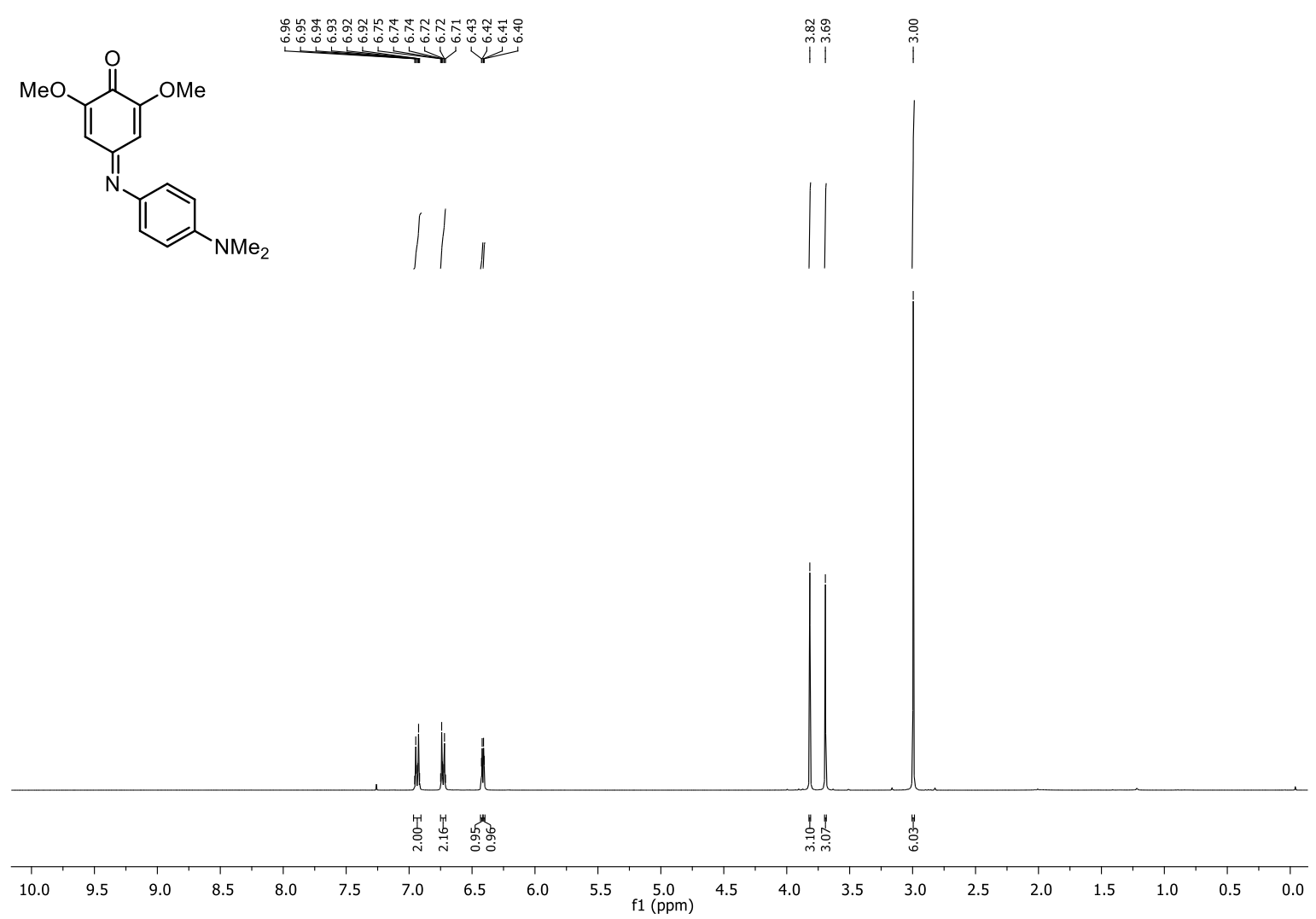

${ }^{13} \mathrm{C}$ - NMR spectrum of compound $10\left(100 \mathrm{MHz}, \mathrm{CDCl}_{3}\right)$

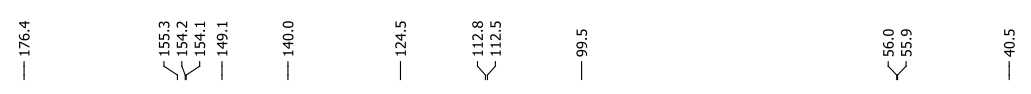
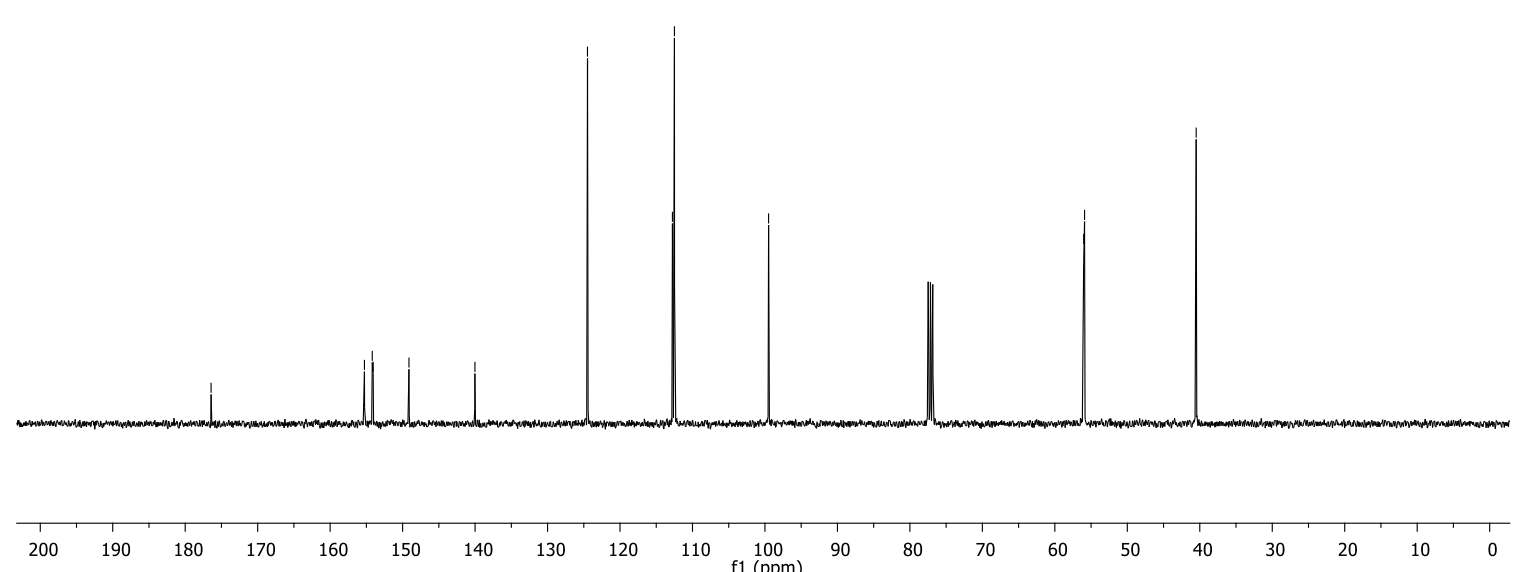
${ }^{1} \mathrm{H}$ - NMR spectrum of compound $11\left(400 \mathrm{MHz}, \mathrm{CDCl}_{3}\right)$
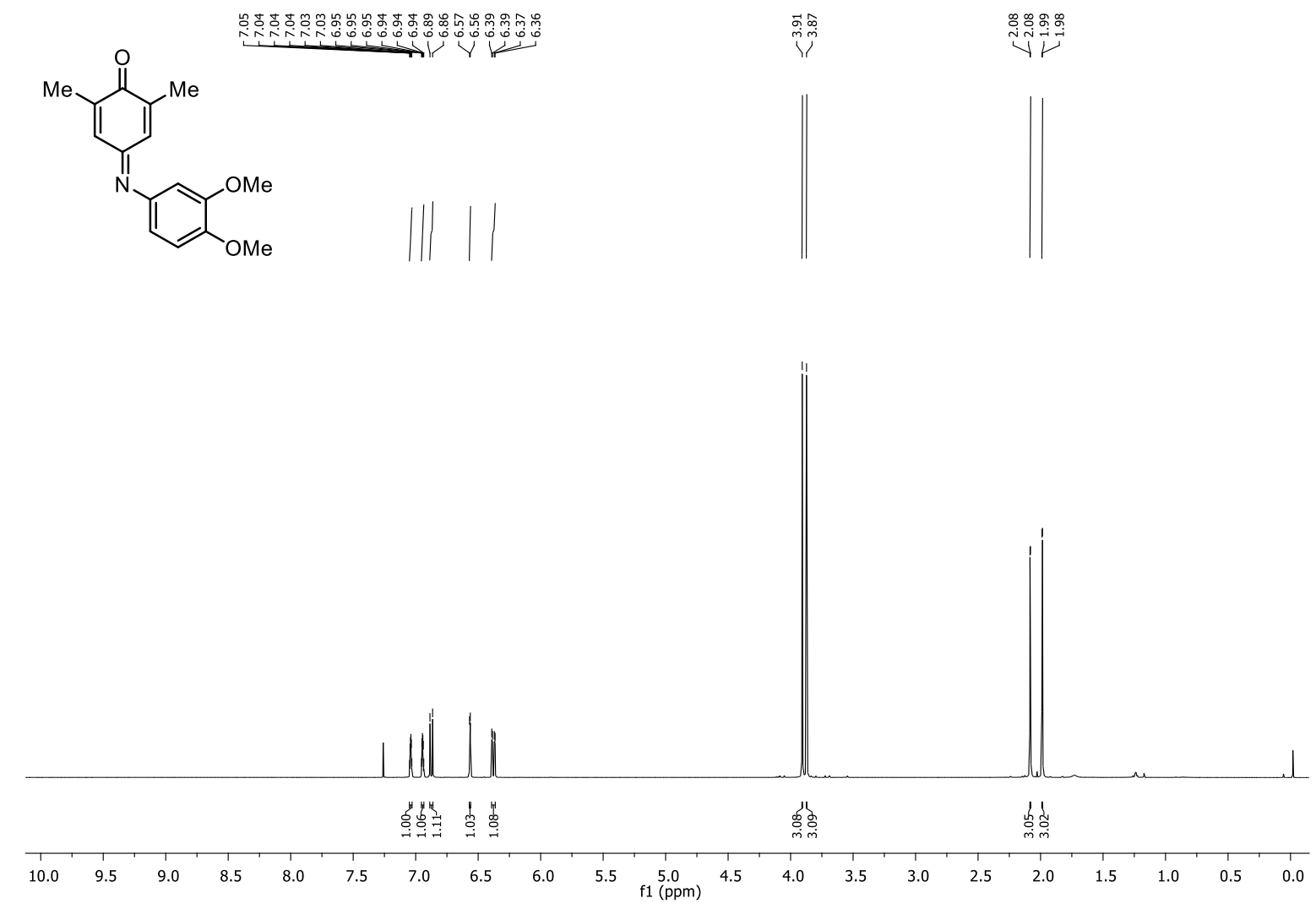

${ }^{13} \mathrm{C}$ - NMR spectrum of compound $11\left(100 \mathrm{MHz}, \mathrm{CDCl}_{3}\right)$

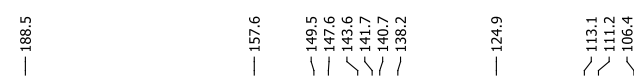

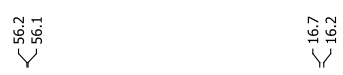

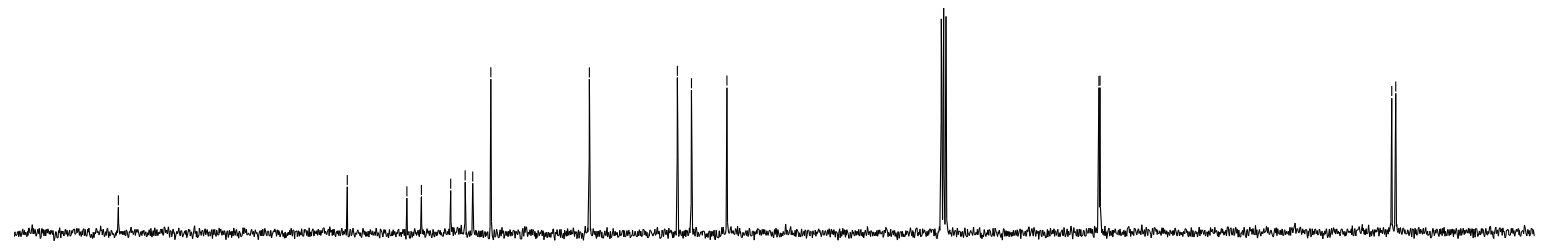

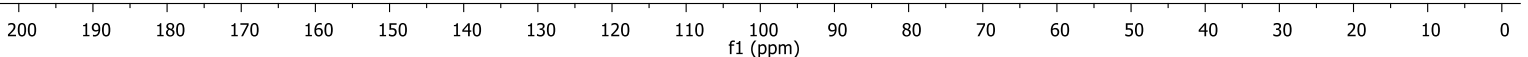


${ }^{1} \mathrm{H}$ - NMR spectrum of compound $12\left(400 \mathrm{MHz}, \mathrm{CDCl}_{3}\right)$
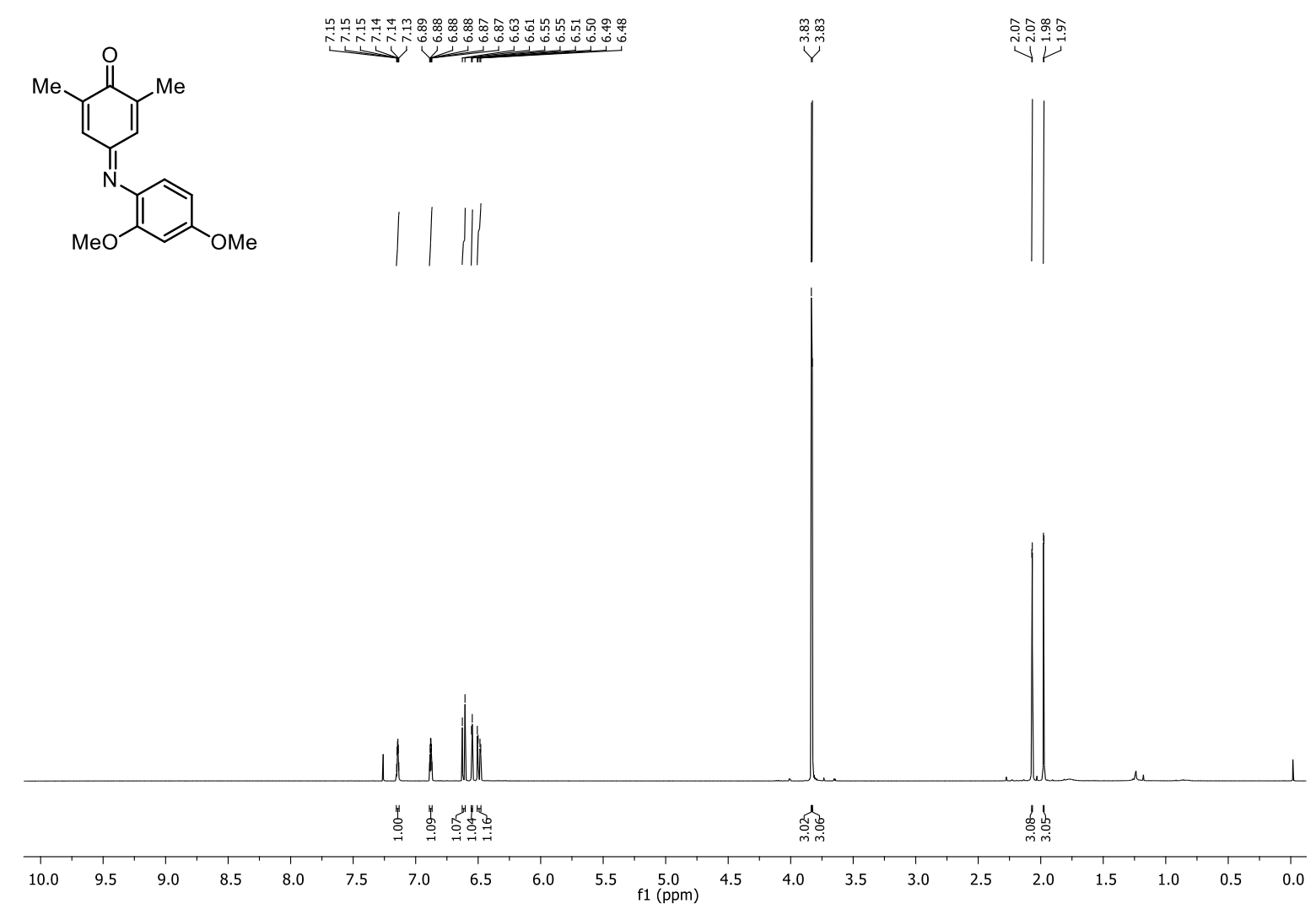

${ }^{13} \mathrm{C}$ - NMR spectrum of compound $12\left(100 \mathrm{MHz}, \mathrm{CDCl}_{3}\right)$

兽

บํ.

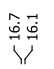

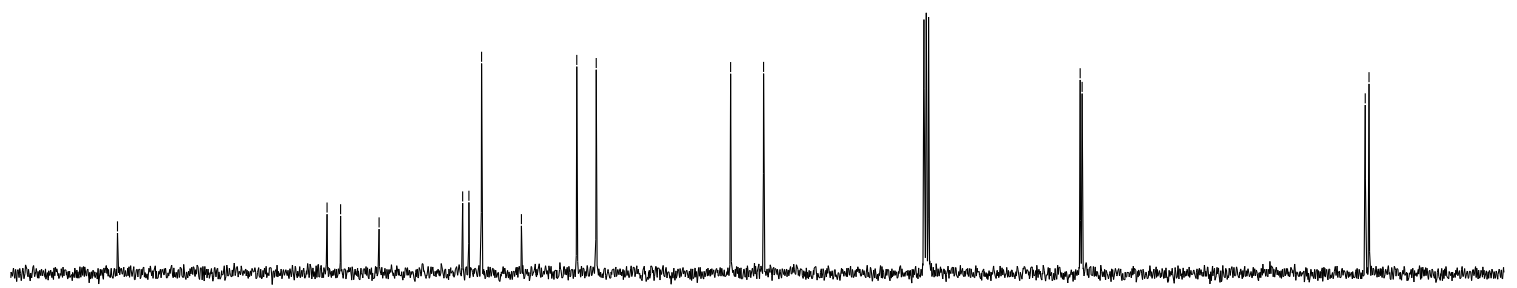


${ }^{1} \mathrm{H}$ - NMR spectrum of compound $13\left(400 \mathrm{MHz}, \mathrm{CDCl}_{3}\right)$
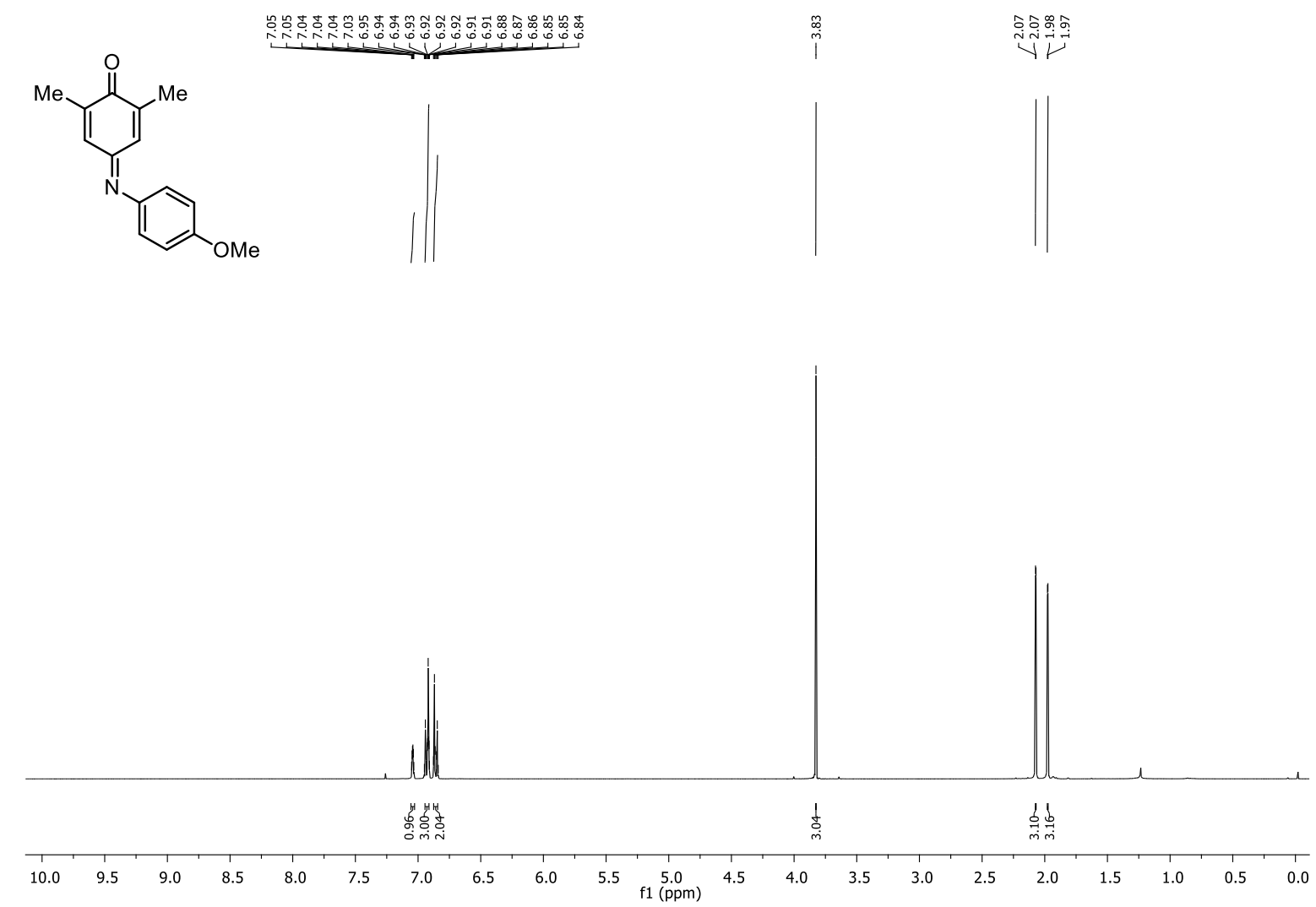

${ }^{13} \mathrm{C}$ - NMR spectrum of compound $13\left(100 \mathrm{MHz}, \mathrm{CDCl}_{3}\right)$

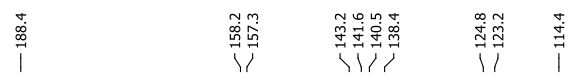
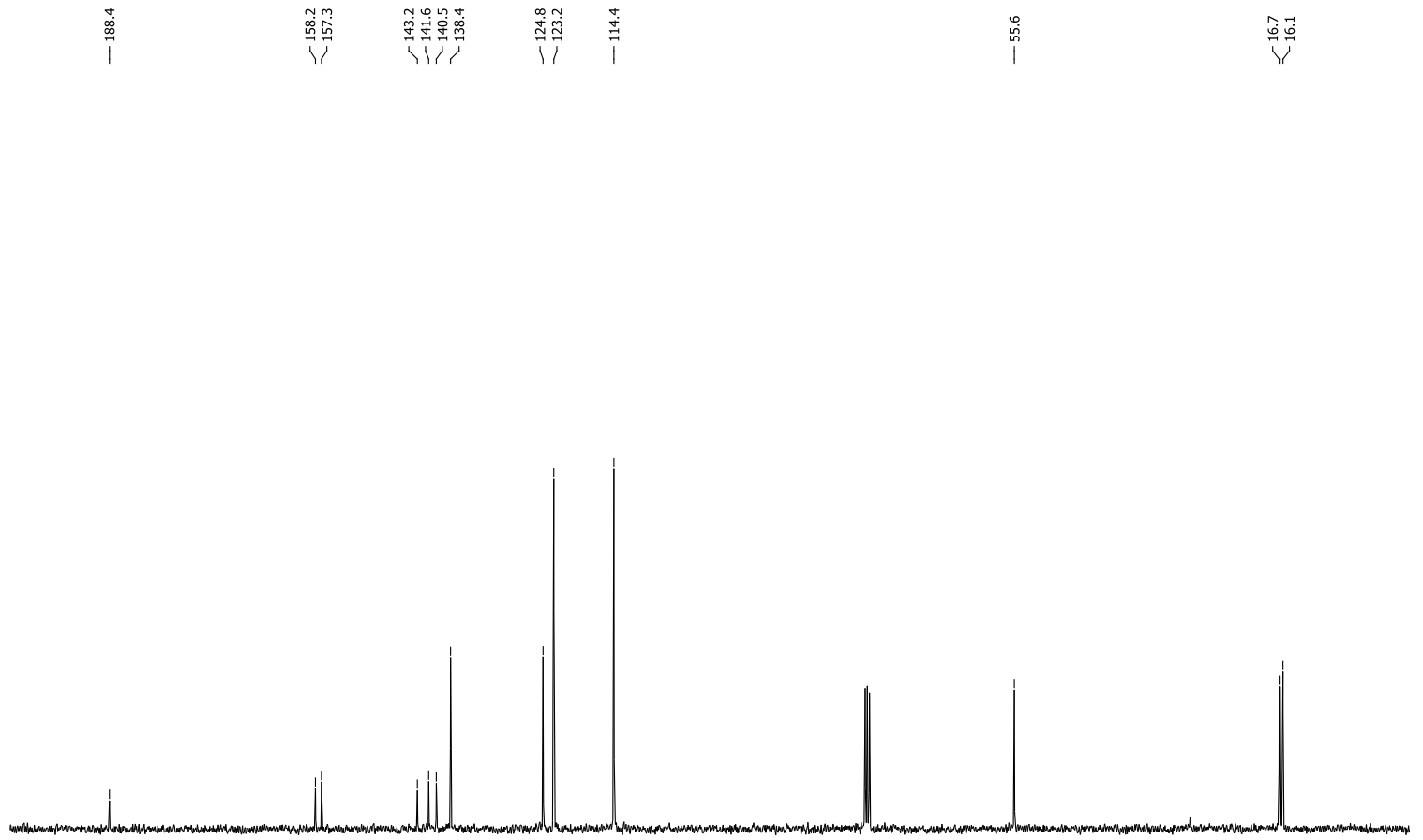

200

$70 \quad 160$

$150 \quad 140$

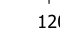

$110 \quad \begin{aligned} & 100 \\ & \mathrm{f} 1(\mathrm{ppm})\end{aligned}$ 
${ }^{1} \mathrm{H}$ - NMR spectrum of compound 14 (400 MHz, Acetone- $\left.d_{6}\right)$<smiles>CC1=CC(=Nc2ccc(O)cc2)C=C(O)C1=O</smiles>
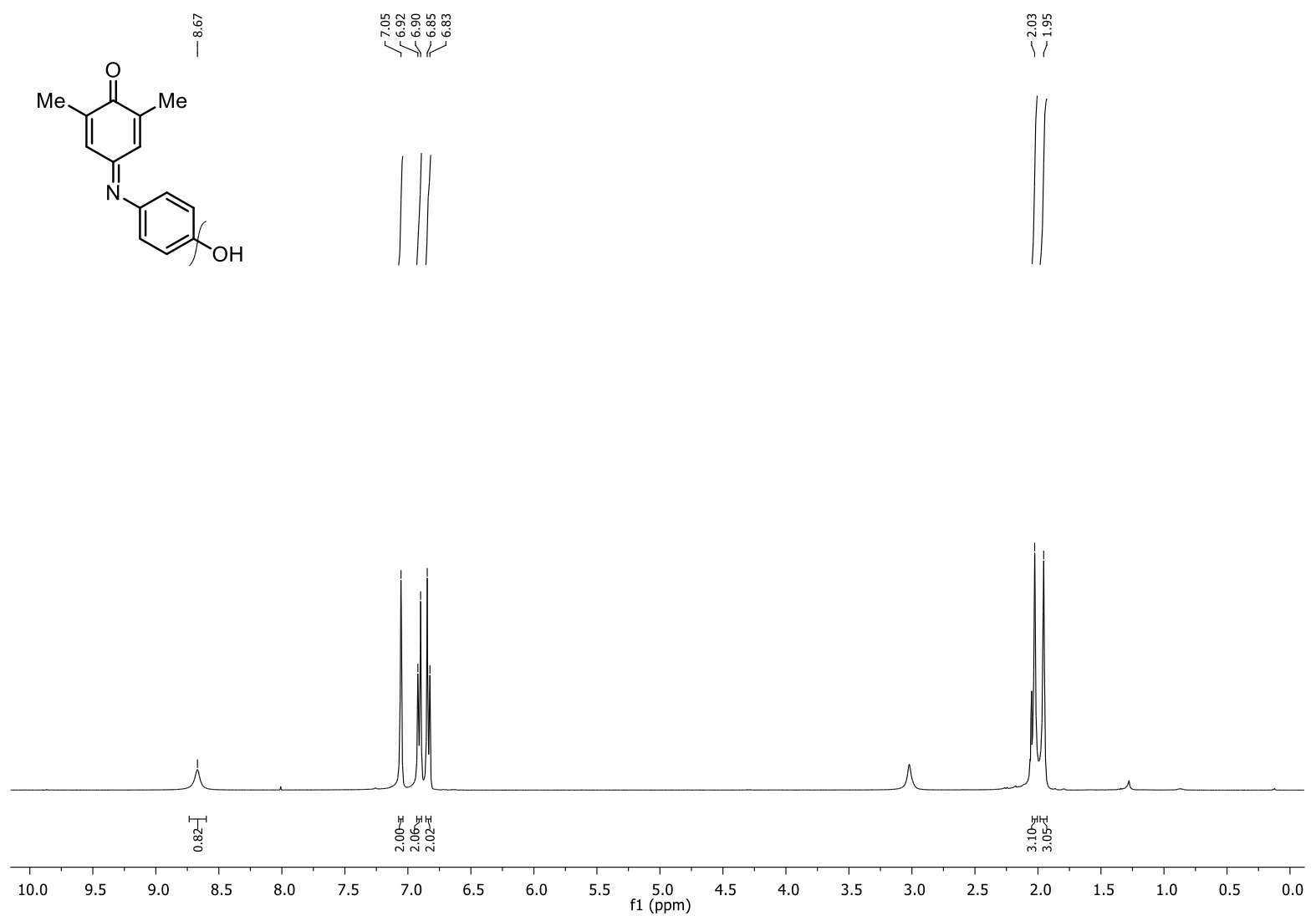

${ }^{13} \mathrm{C}$ - NMR spectrum of compound $14\left(100 \mathrm{MHz}\right.$, Acetone- $\left.d_{6}\right)$

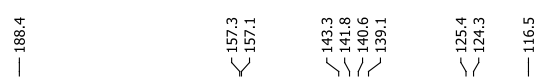

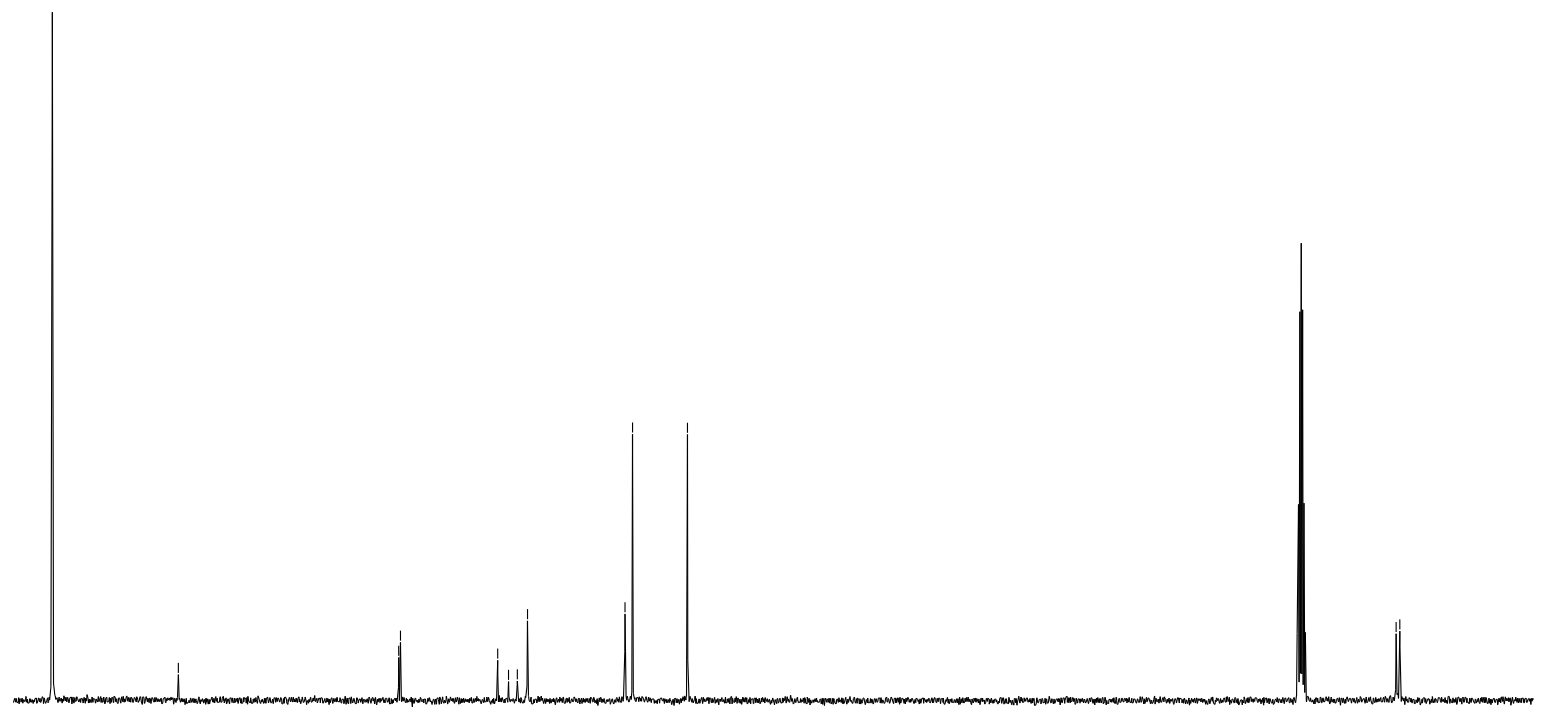

$\begin{array}{llllllllllllllllllllllllll}1 & 210 & 200 & 190 & 180 & 170 & 160 & 150 & 140 & 130 & 120 & \underset{\mathrm{f} 1(\mathrm{ppm})}{110} & 90 & 80 & 70 & 60 & 50 & 40 & 30 & 20 & 10 & 0\end{array}$ 
${ }^{1} \mathrm{H}$ - NMR spectrum of compound $15\left(400 \mathrm{MHz}, \mathrm{CDCl}_{3}\right)$
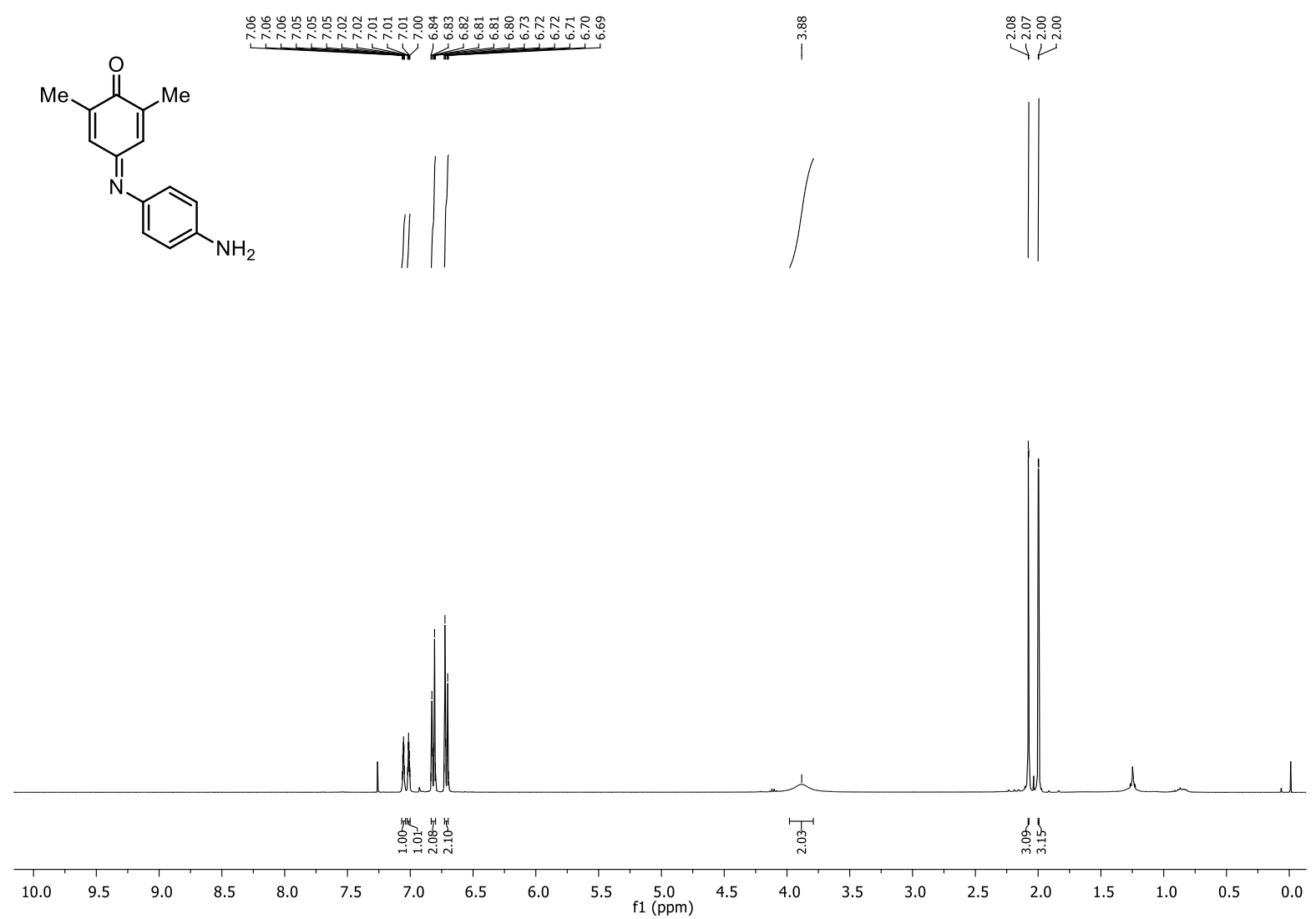

${ }^{13} \mathrm{C}$ - NMR spectrum of compound $15\left(100 \mathrm{MHz}, \mathrm{CDCl}_{3}\right)$

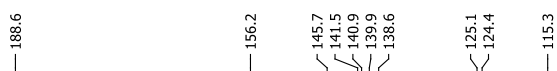

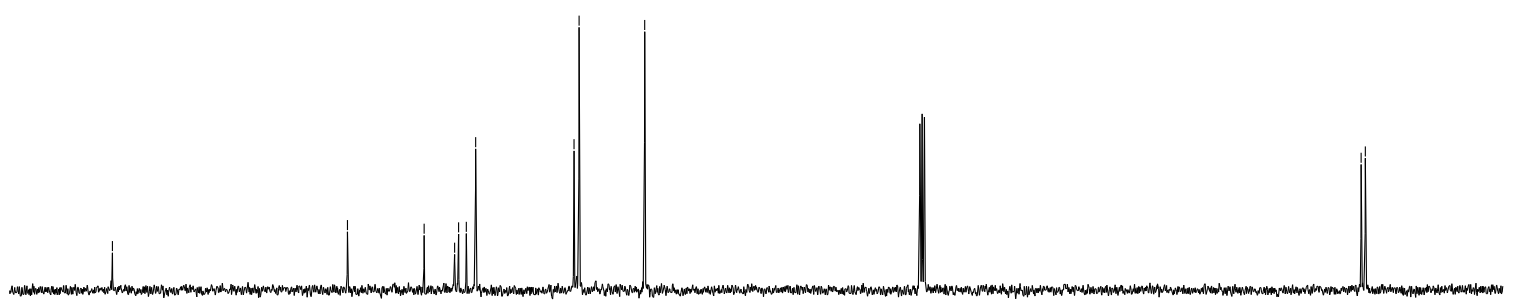

200 $\begin{array}{llllllllll}190 & 180 & 170 & 160 & 150 & 140 & 130 & 120 & 110 & \begin{array}{l}100 \\ \mathrm{f} 1(\mathrm{ppm})\end{array}\end{array}$ 
${ }^{1} \mathrm{H}$ - NMR spectrum of compound $16\left(400 \mathrm{MHz}, \mathrm{CDCl}_{3}\right)$

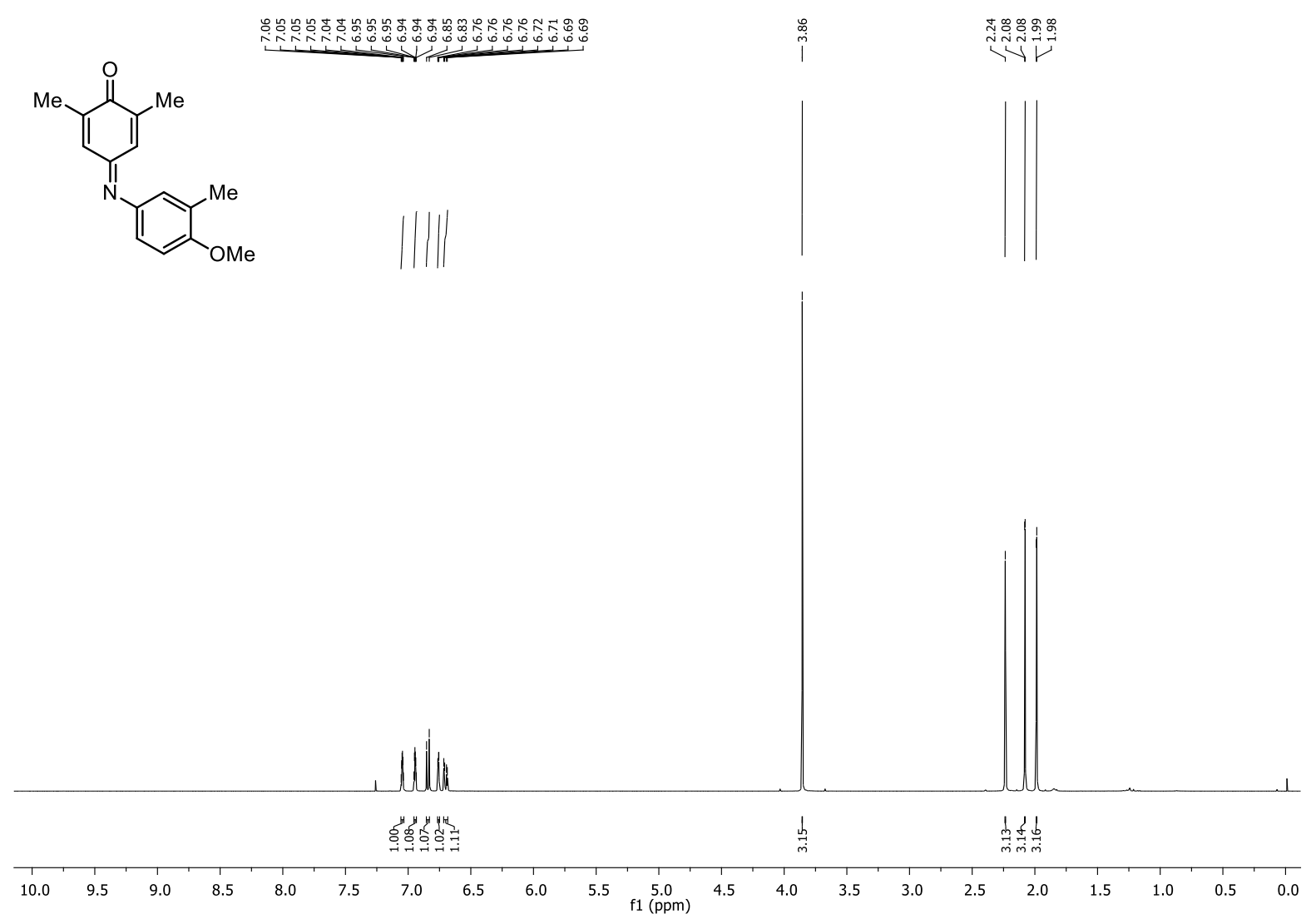

${ }^{13} \mathrm{C}$ - NMR spectrum of compound $16\left(100 \mathrm{MHz}, \mathrm{CDCl}_{3}\right)$
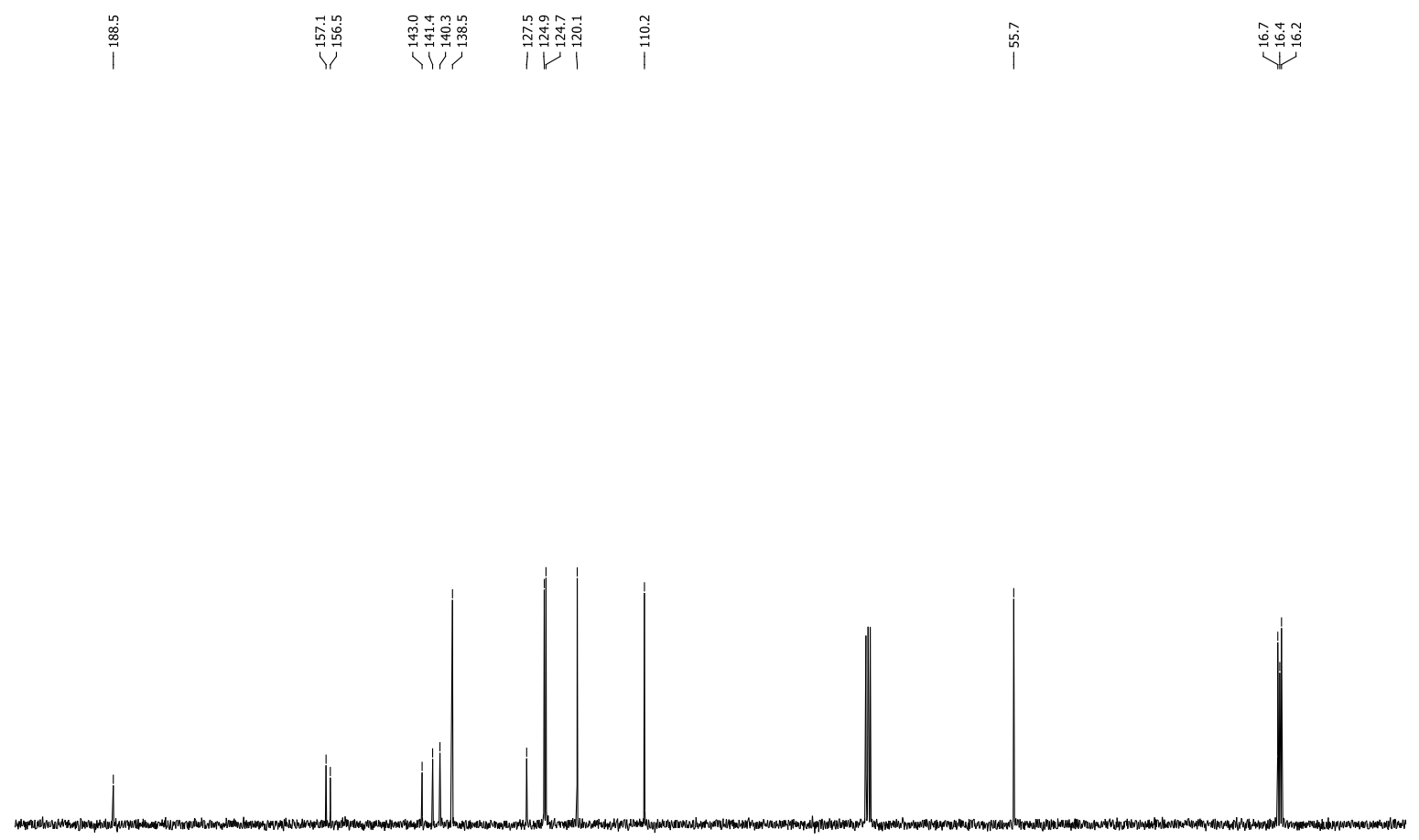

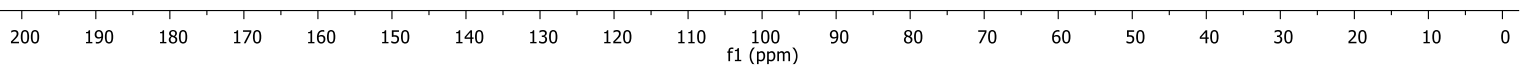


${ }^{1} \mathrm{H}$ - NMR spectrum of compound $17\left(400 \mathrm{MHz}, \mathrm{CDCl}_{3}\right)$<smiles>COC1=CC(=Nc2ccc(OC)cc2[N+](=O)[O-])C=C([N+](=O)[O-])C1=O</smiles>

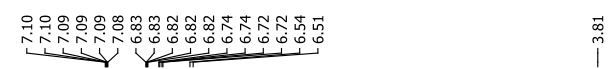

บำ
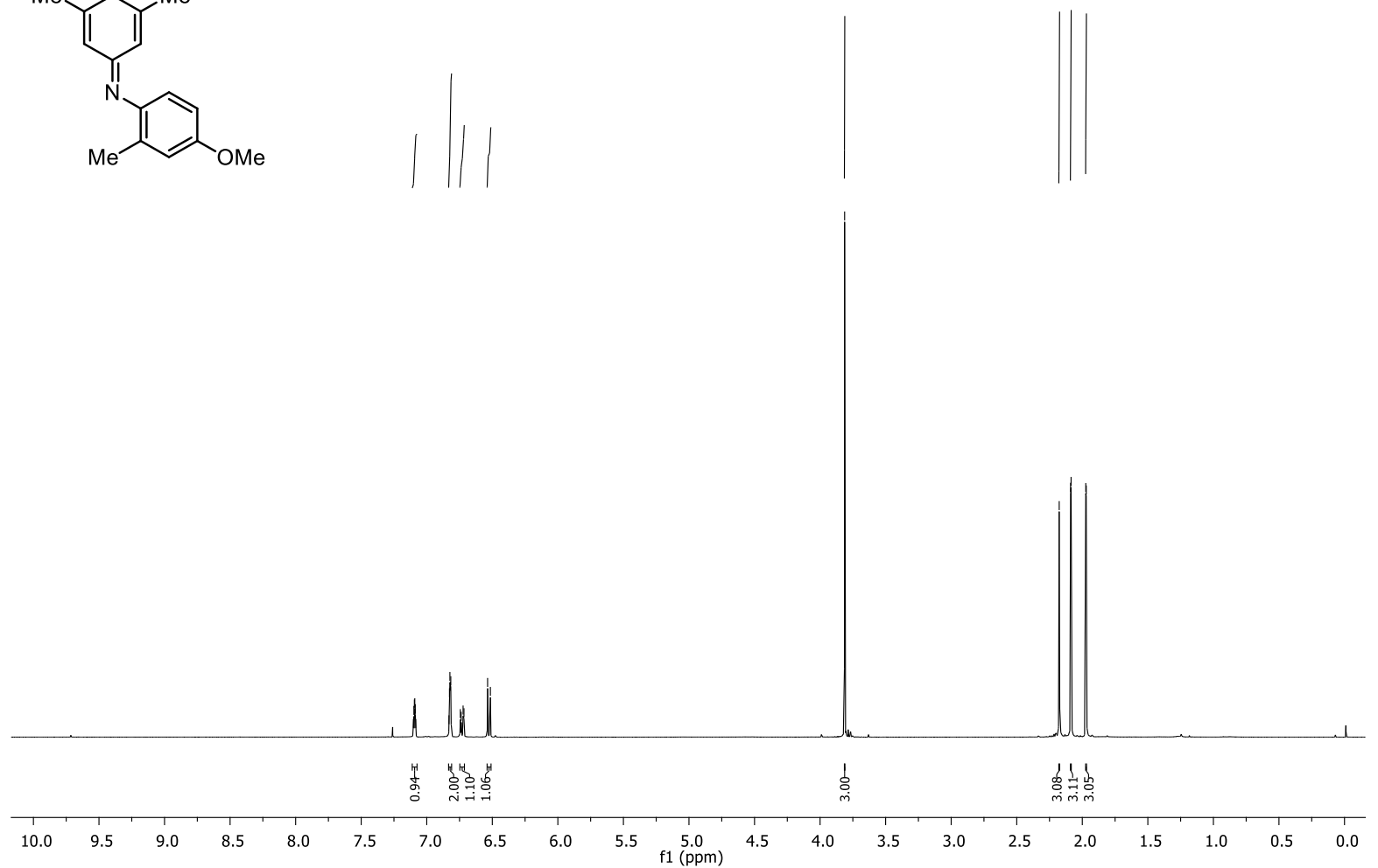

${ }^{13} \mathrm{C}$ - NMR spectrum of compound $17\left(100 \mathrm{MHz}, \mathrm{CDCl}_{3}\right)$

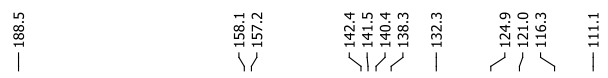

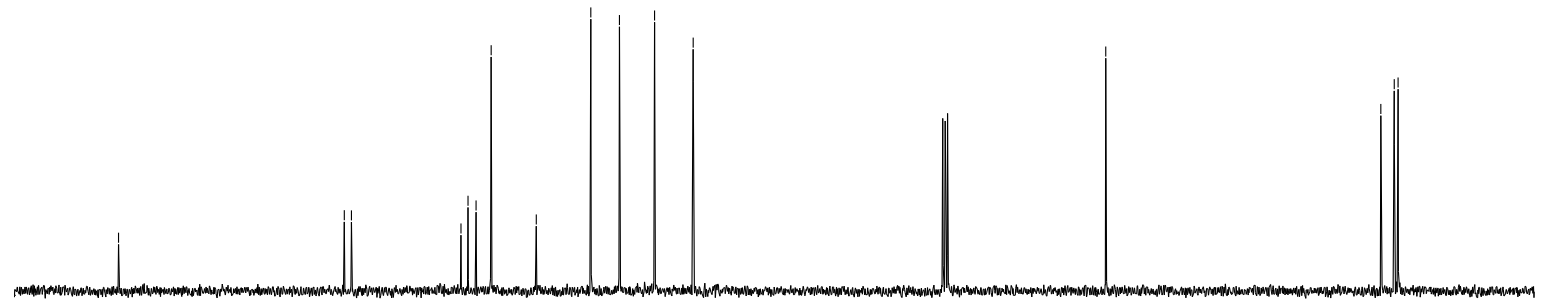

$\begin{array}{lllllllllllllllllllllll}200 & 190 & 180 & 170 & 160 & 150 & 140 & 130 & 120 & 110 & \begin{array}{c}100 \\ \mathrm{f} 1(\mathrm{ppm})\end{array} & 90 & 80 & 70 & 60 & 50 & 40 & 30 & 20 & 10 & 0\end{array}$ 
${ }^{1} \mathrm{H}$ - NMR spectrum of compound $18\left(400 \mathrm{MHz}, \mathrm{CDCl}_{3}\right)$<smiles>COC1=CC(=Nc2ccc(OC)c(OC)c2)C(OC)=CC1=O</smiles>
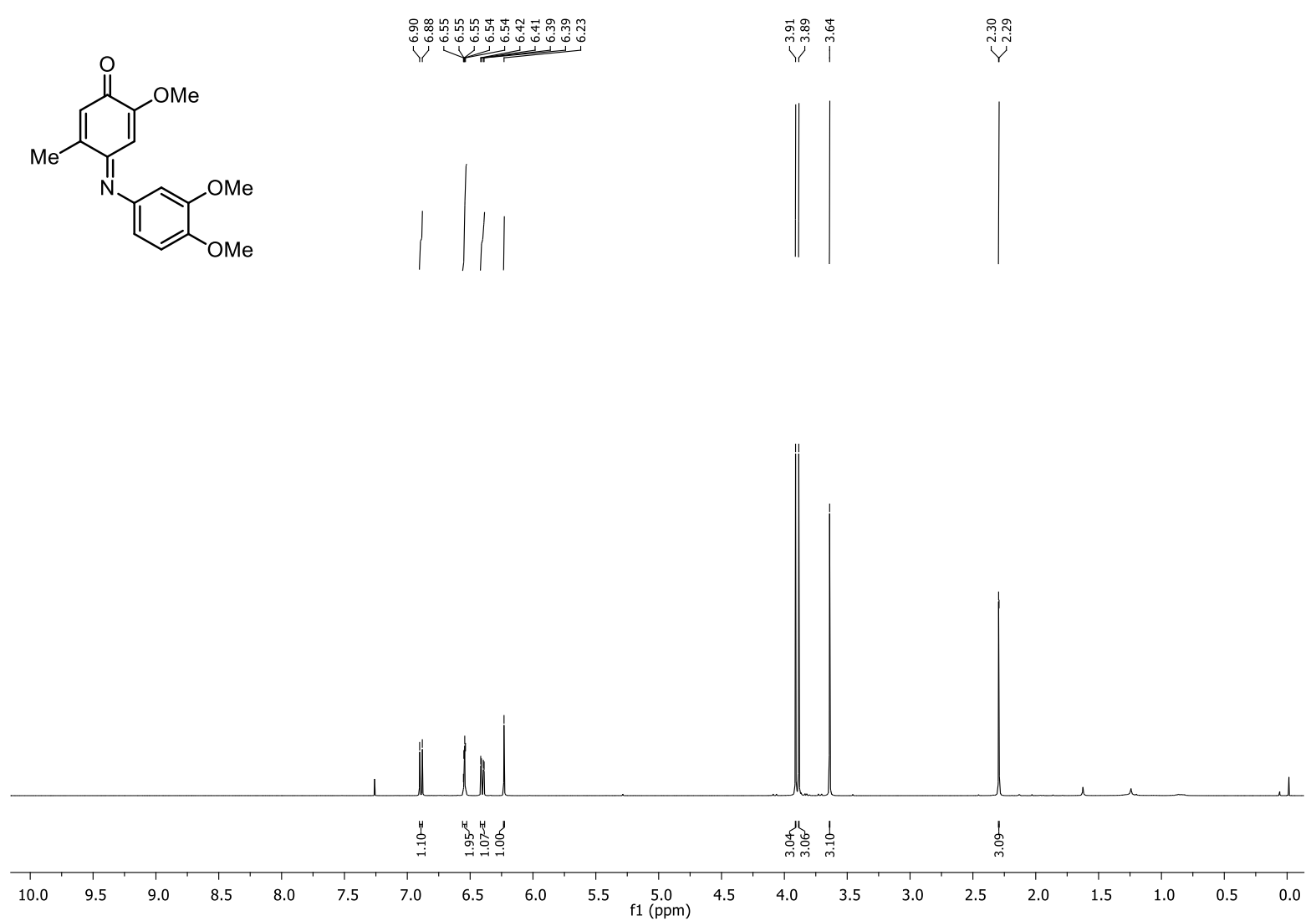

${ }^{13} \mathrm{C}$ - NMR spectrum of compound $18\left(100 \mathrm{MHz}, \mathrm{CDCl}_{3}\right)$

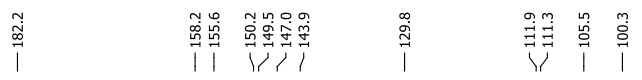

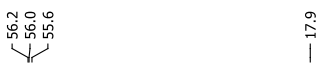

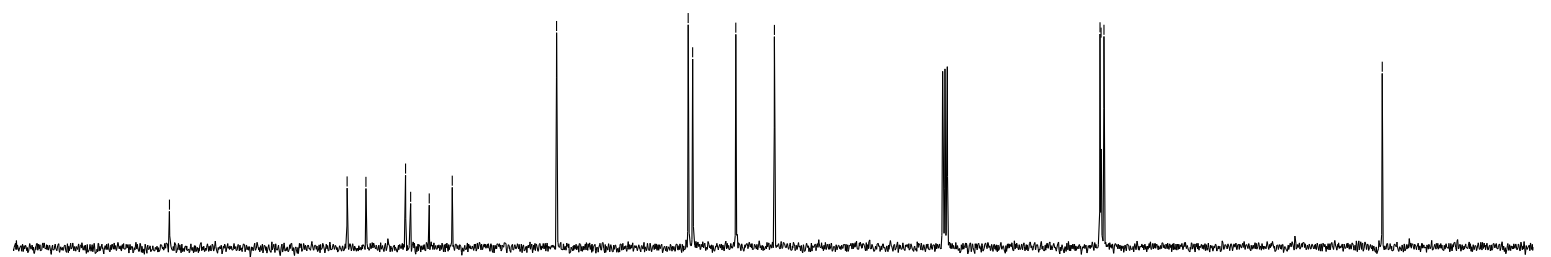

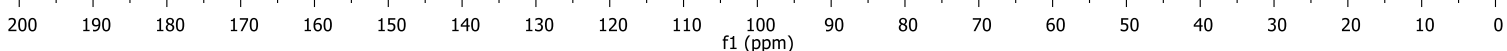


${ }^{1} \mathrm{H}$ - NMR spectrum of compound $19\left(400 \mathrm{MHz}, \mathrm{CDCl}_{3}\right)$<smiles>COc1ccc(N=C2C=C(C)C(=O)C=C2C)c(OC)c1</smiles>

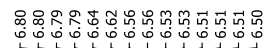

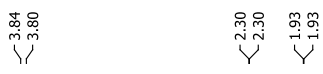
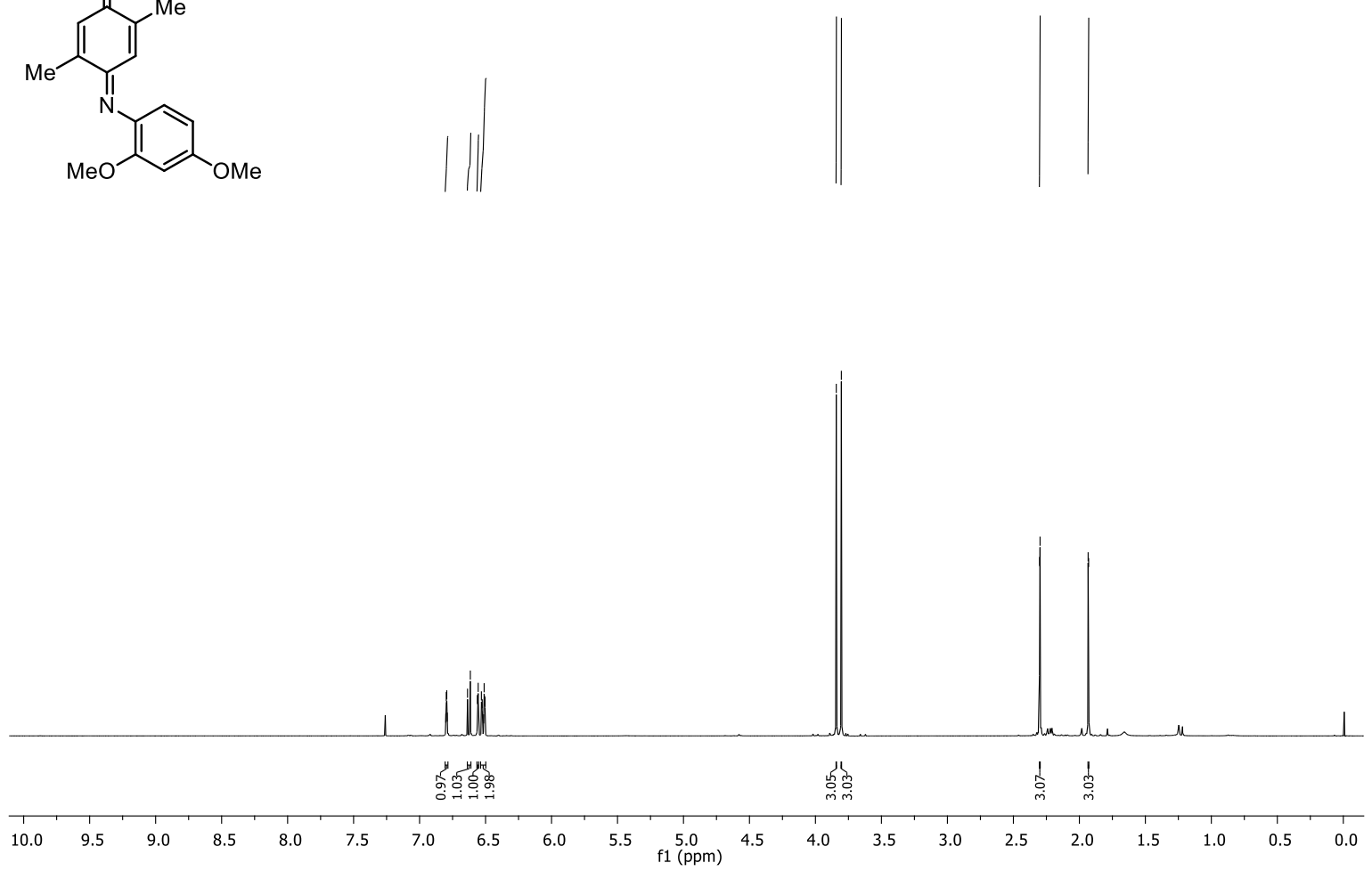

${ }^{13} \mathrm{C}$ - NMR spectrum of compound $19\left(100 \mathrm{MHz}, \mathrm{CDCl}_{3}\right)$

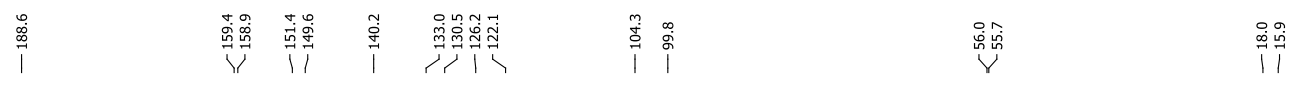

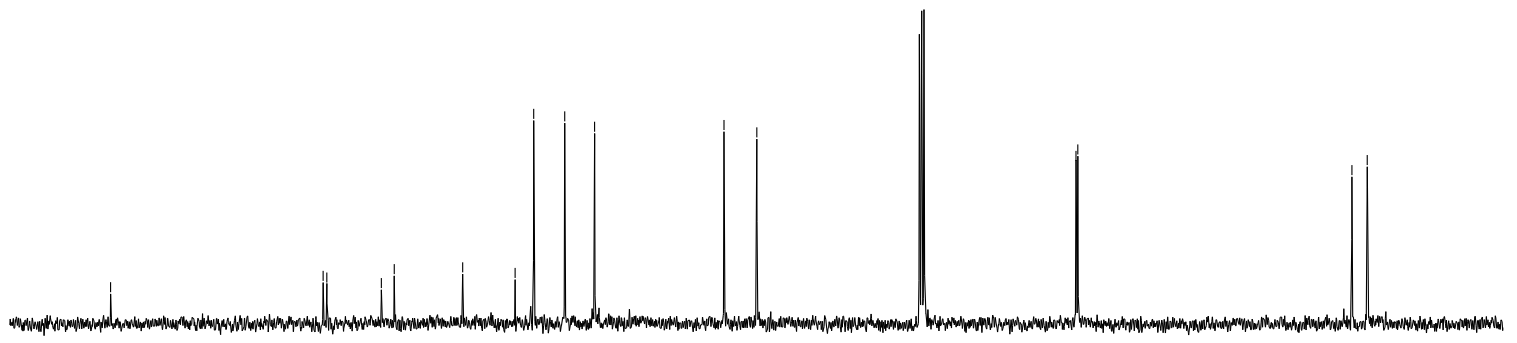

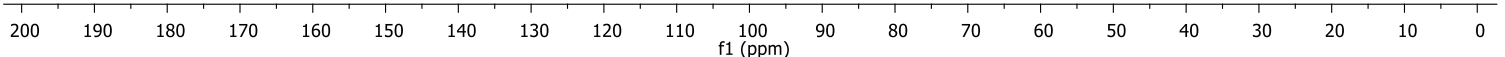


${ }^{1} \mathrm{H}-\mathrm{NMR}$ spectrum of compound $20\left(500 \mathrm{MHz}, \mathrm{DMSO}-d_{6}, 347 \mathrm{~K}\right)$<smiles>Nc1cc2nc3ccccc3oc-2cc1=O</smiles>

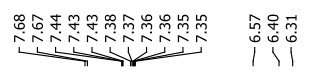
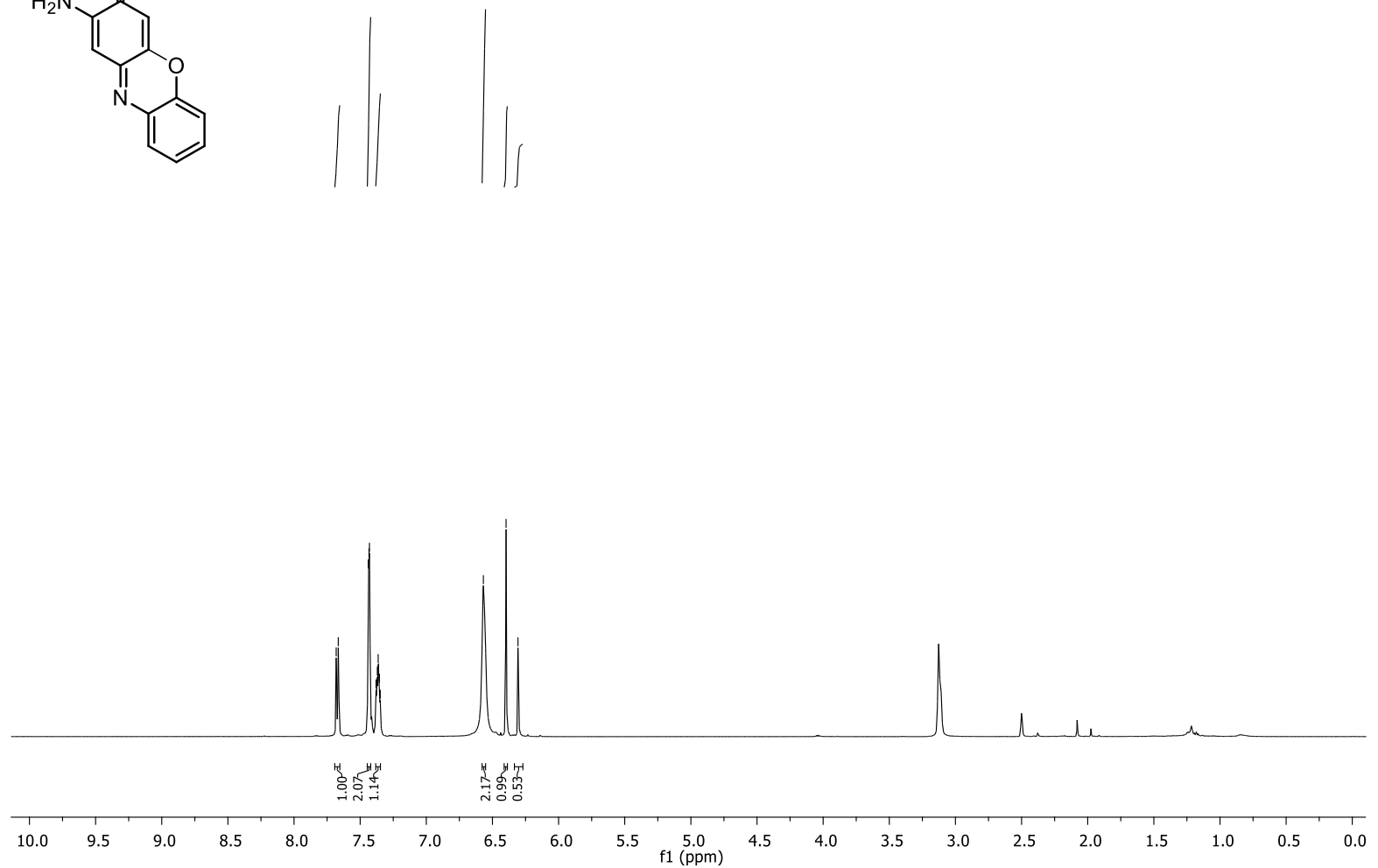

${ }^{13} \mathrm{C}$ - NMR spectrum of compound $20\left(125 \mathrm{MHz}, \mathrm{DMSO}-d_{6}, 347 \mathrm{~K}\right)$

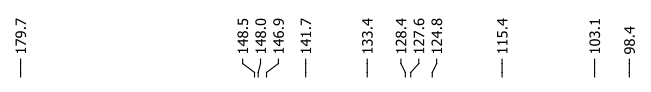

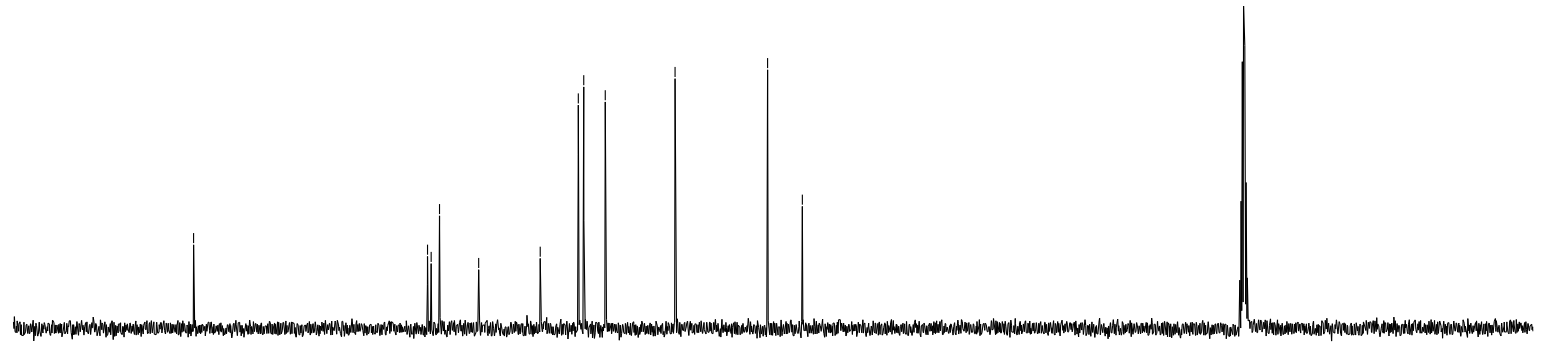

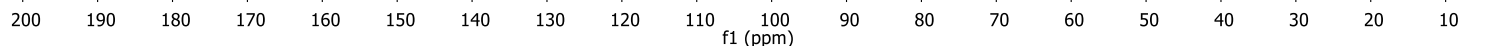


${ }^{1} \mathrm{H}$ - NMR spectrum of compound $21\left(400 \mathrm{MHz}, \mathrm{CDCl}_{3}\right)$<smiles>CC(C)(C)c1ccc2oc3cc(=O)c(N)cc-3nc2c1</smiles>

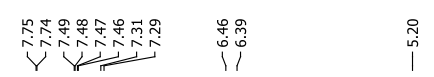

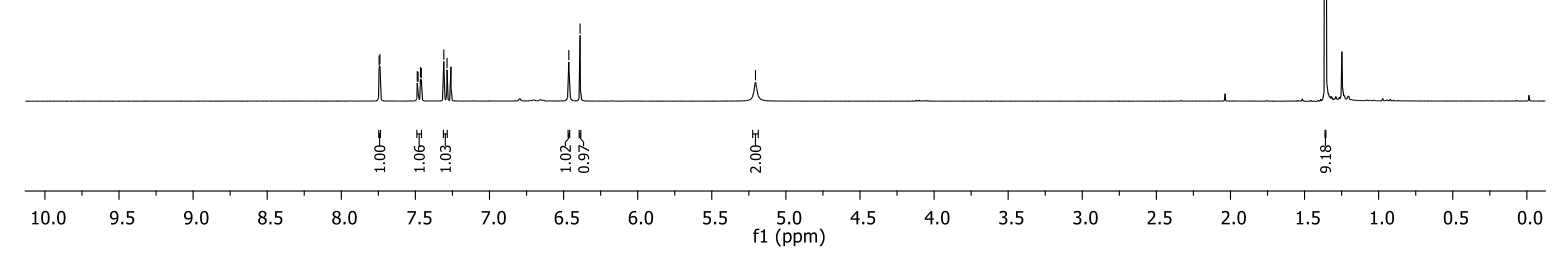

${ }^{13} \mathrm{C}$ - NMR spectrum of compound $21\left(100 \mathrm{MHz}, \mathrm{CDCl}_{3}\right)$

lli

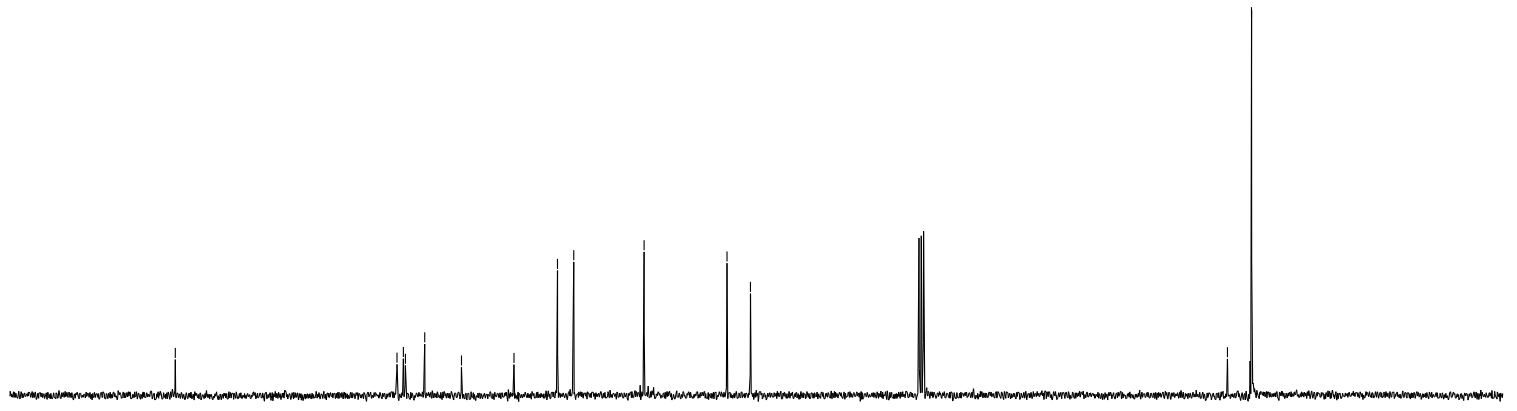


${ }^{1} \mathrm{H}$ - NMR spectrum of compound $22\left(400 \mathrm{MHz}, \mathrm{CDCl}_{3}\right)$<smiles>COc1ccc(N=C2C=CC(=O)C=C2)c(OC)c1</smiles>

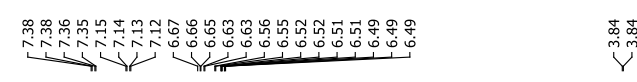

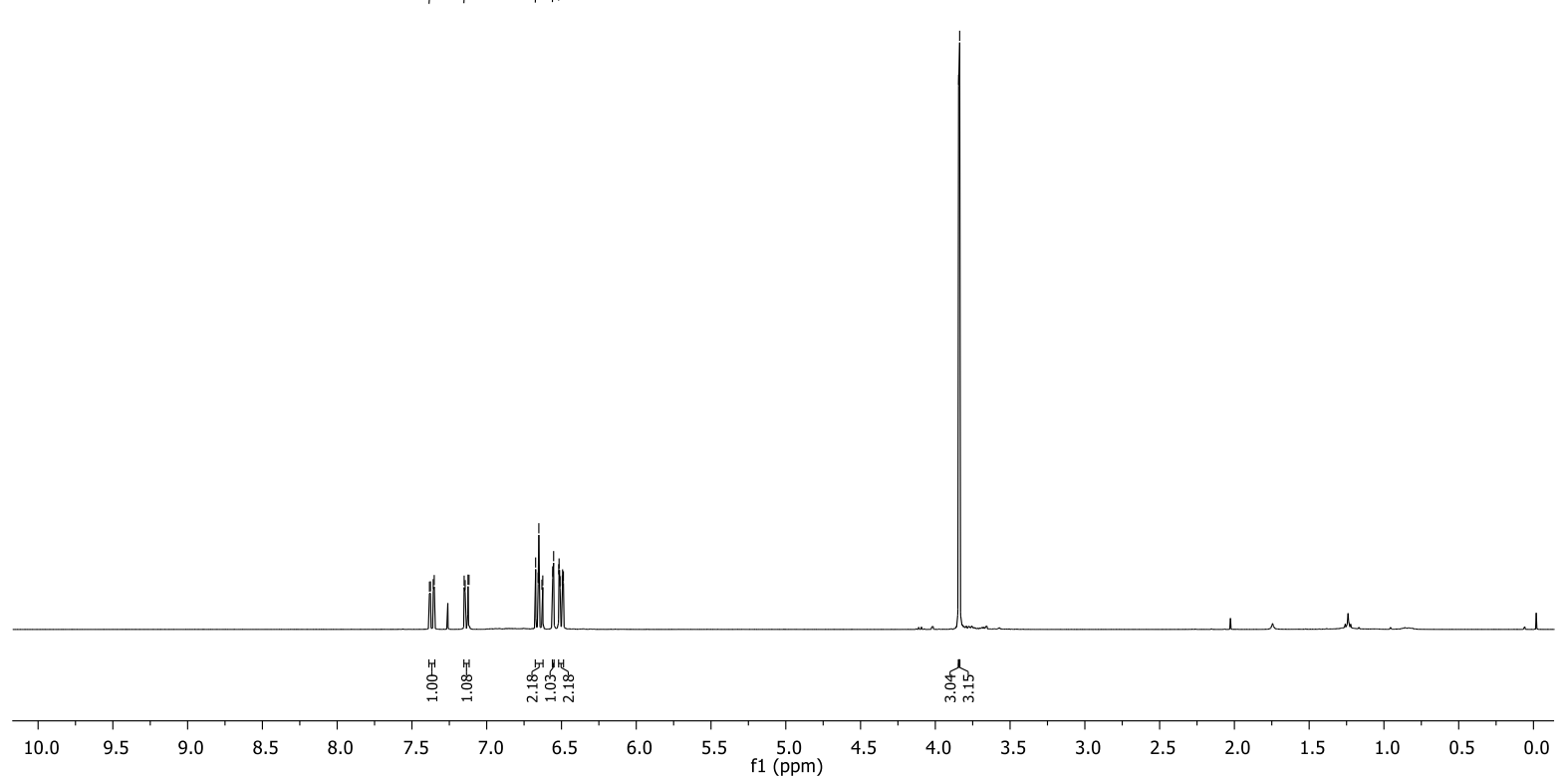

${ }^{13} \mathrm{C}$ - NMR spectrum of compound $22\left(100 \mathrm{MHz}, \mathrm{CDCl}_{3}\right)$ إ.
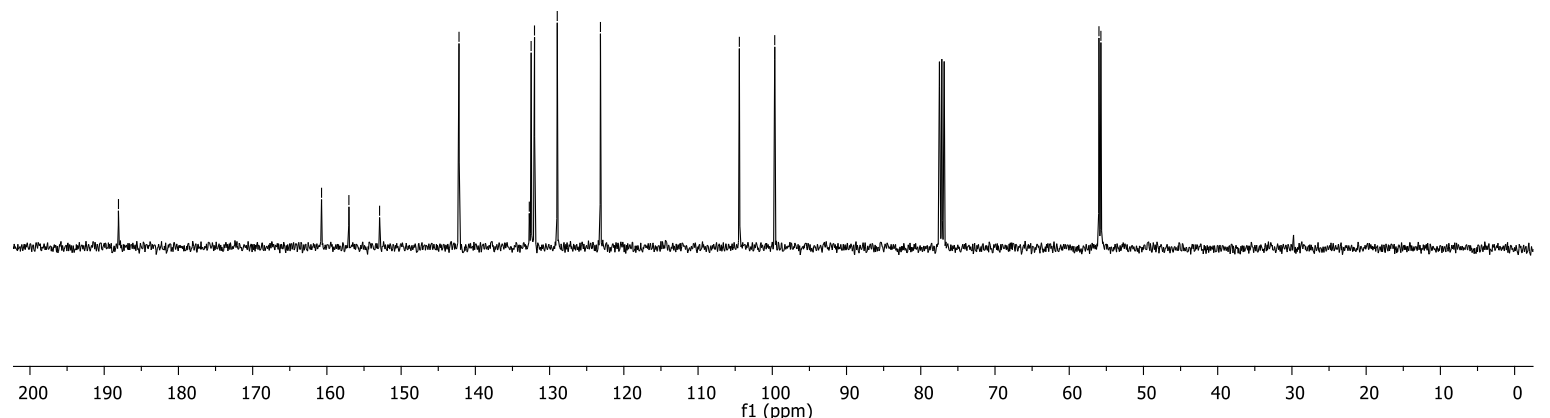
${ }^{1} \mathrm{H}$ - NMR spectrum of two isomeric conformations of compound $23\left(400 \mathrm{MHz}, \mathrm{CDCl}_{3}\right)$
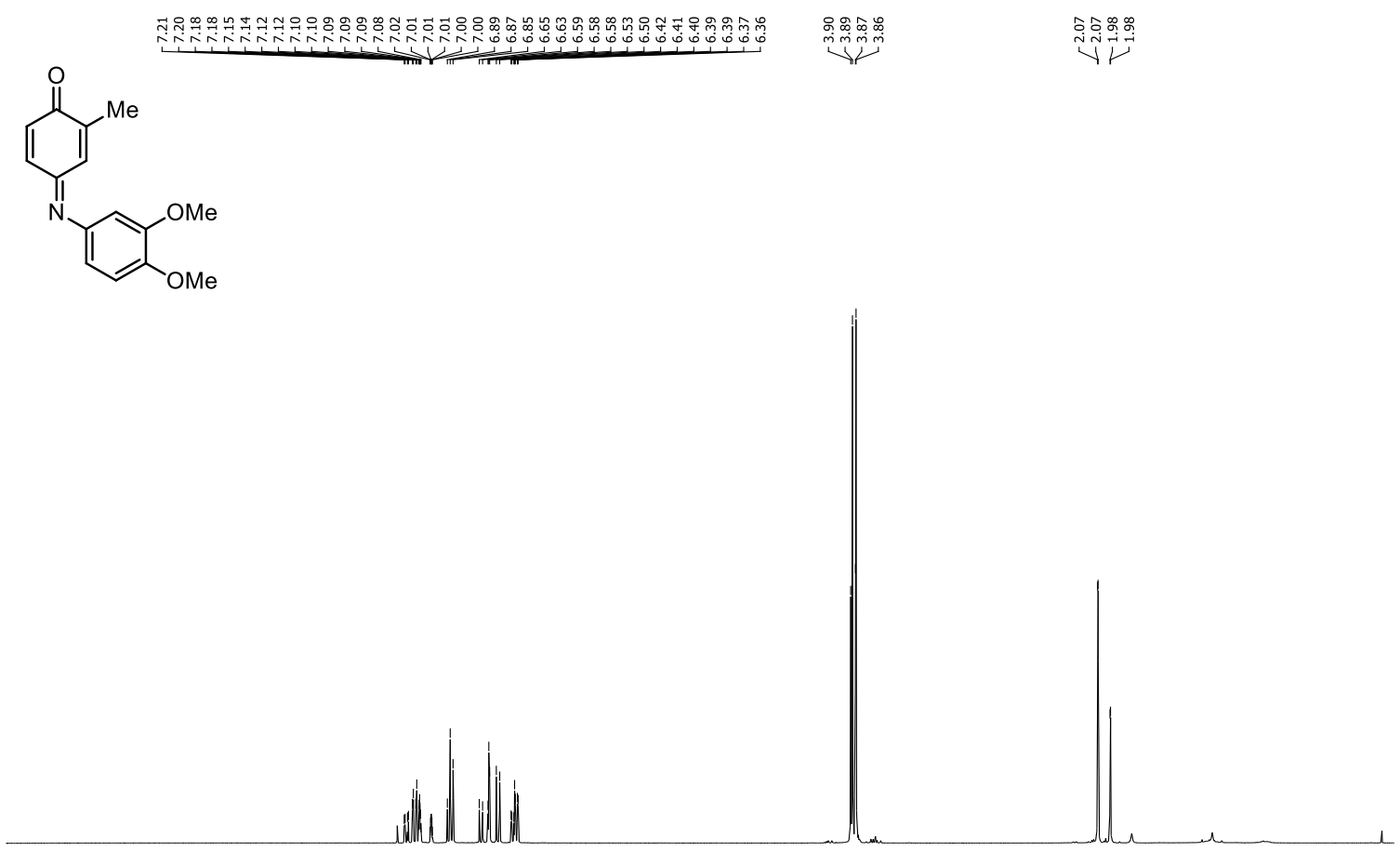

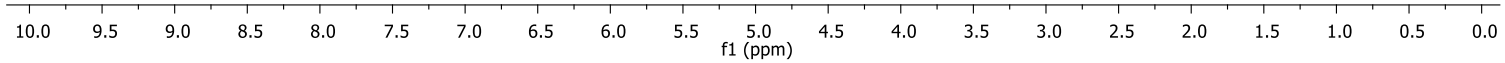

${ }^{13} \mathrm{C}$ - NMR spectrum of two isomeric conformations of compound $23\left(100 \mathrm{MHz}, \mathrm{CDCl}_{3}\right)$

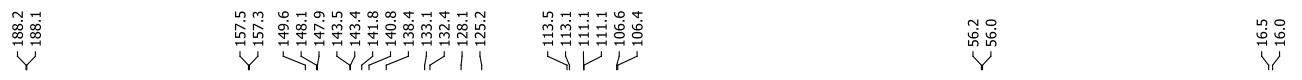

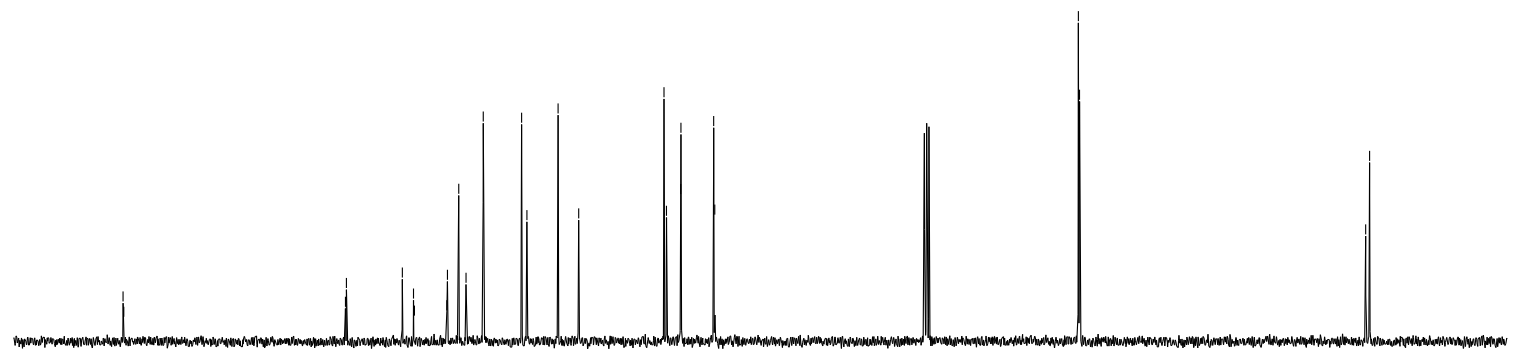


${ }^{1} \mathrm{H}$ - NMR spectrum of compound $24\left(400 \mathrm{MHz}, \mathrm{CDCl}_{3}\right)$
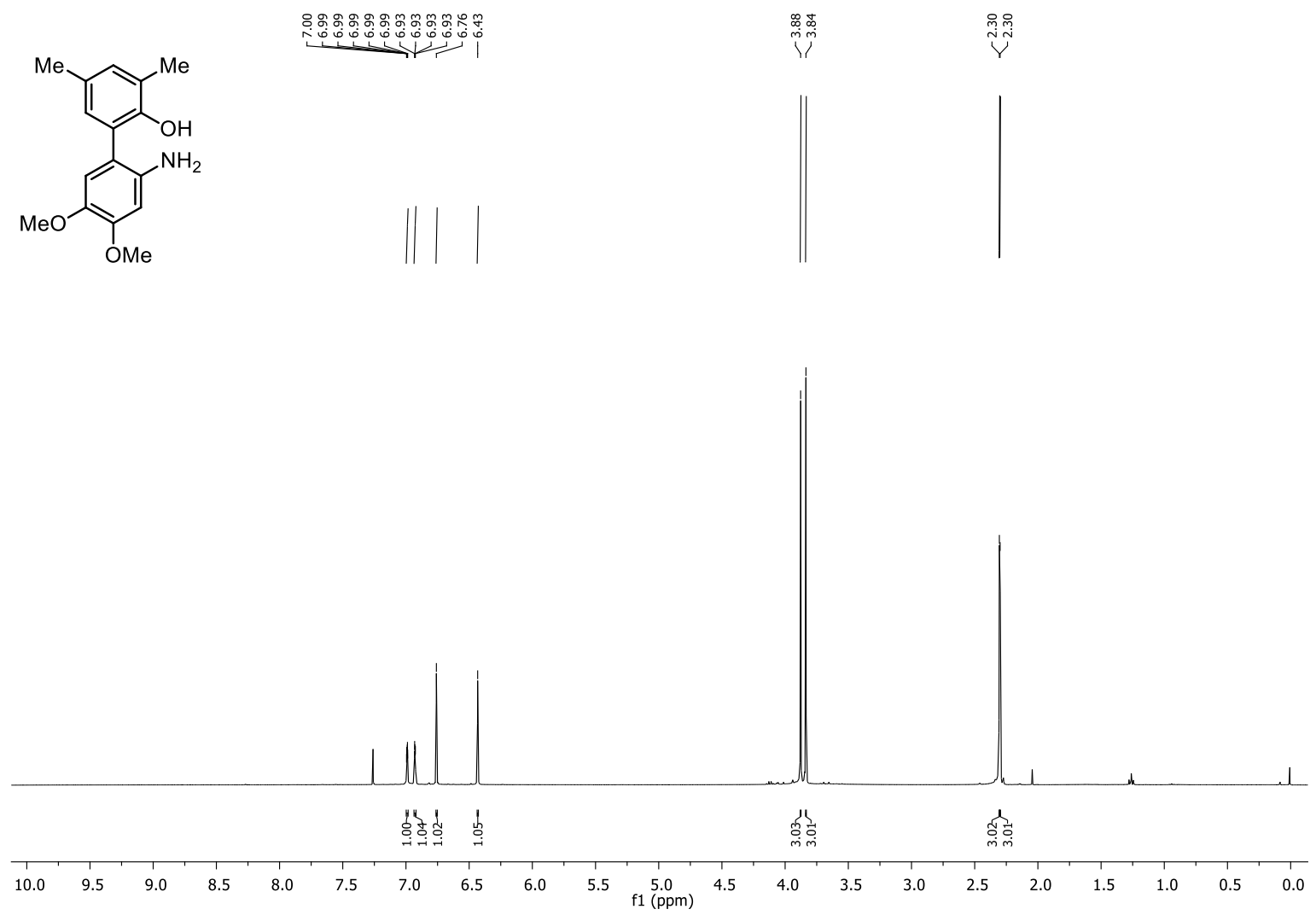

${ }^{13} \mathrm{C}$ - NMR spectrum of compound $24\left(100 \mathrm{MHz}, \mathrm{CDCl}_{3}\right)$

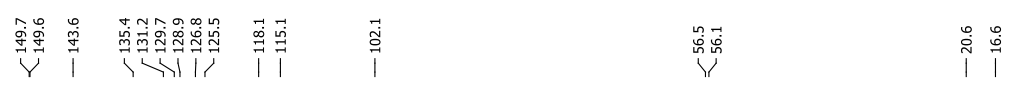

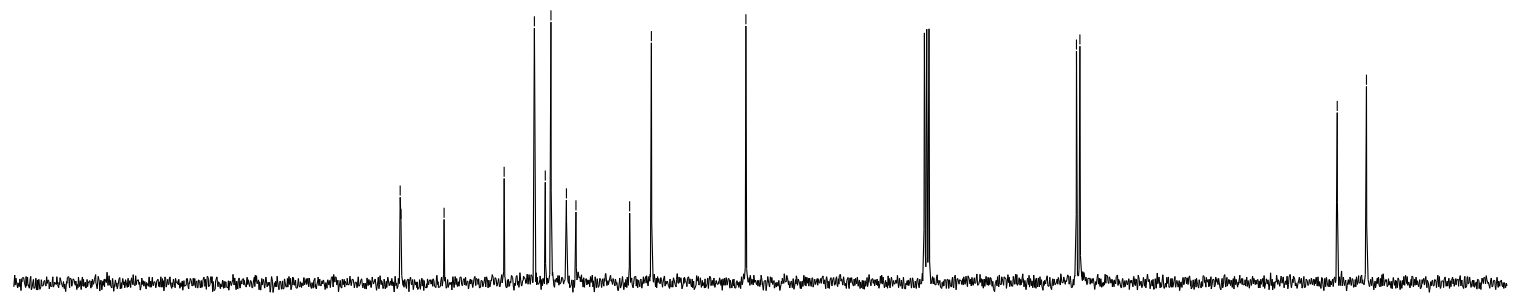

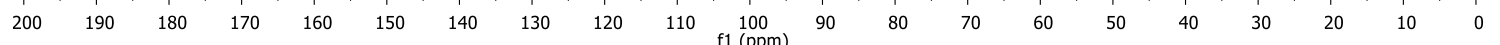


${ }^{1} \mathrm{H}$ - NMR spectrum of compound $25\left(400 \mathrm{MHz}, \mathrm{CDCl}_{3}\right)$
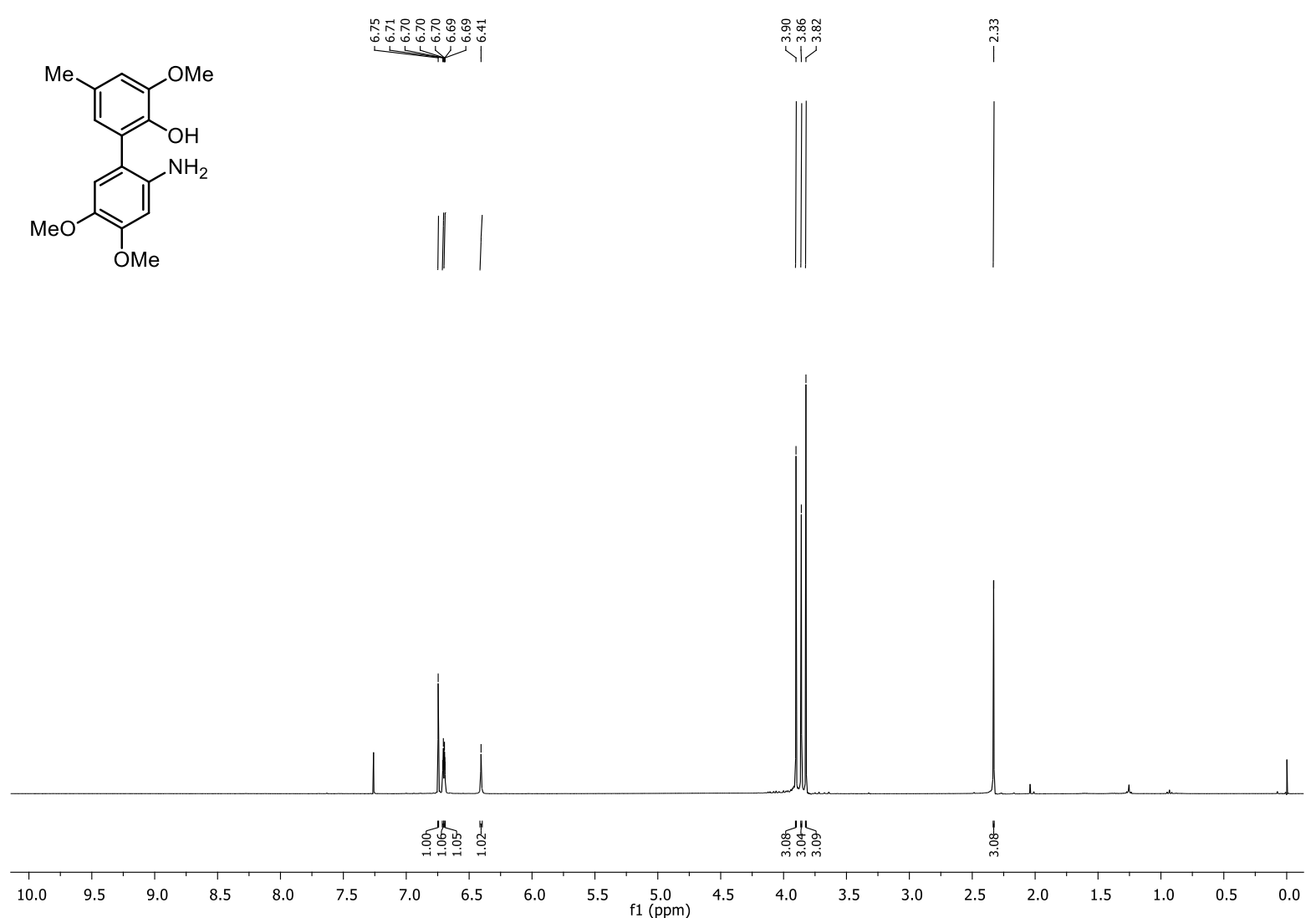

${ }^{13} \mathrm{C}$ - NMR spectrum of compound $25\left(100 \mathrm{MHz}, \mathrm{CDCl}_{3}\right)$
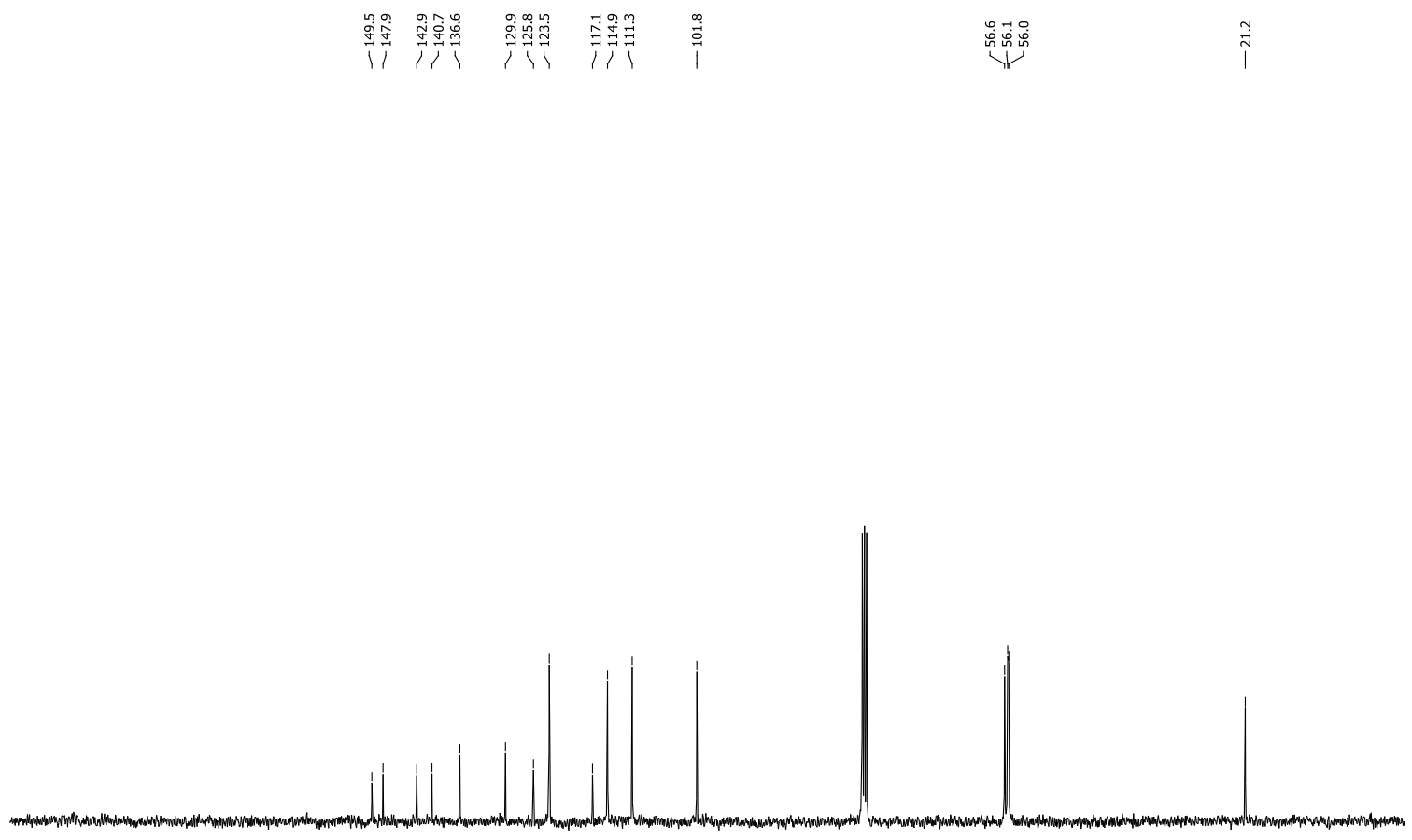

200

190

$\begin{array}{lllll}170 & 160 & 150 & 140 & 130\end{array}$

$120 \quad 110 \begin{array}{cc}100 \\ f 1(\mathrm{ppm})\end{array}$ 
${ }^{1} \mathrm{H}$ - NMR spectrum of compound $26\left(400 \mathrm{MHz}, \mathrm{CDCl}_{3}\right)$<smiles>COc1cc(-c2c(N)cc(OC)cc2O)cc([N+](=O)[O-])c1</smiles>

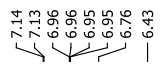

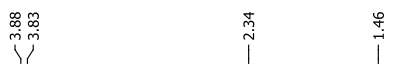
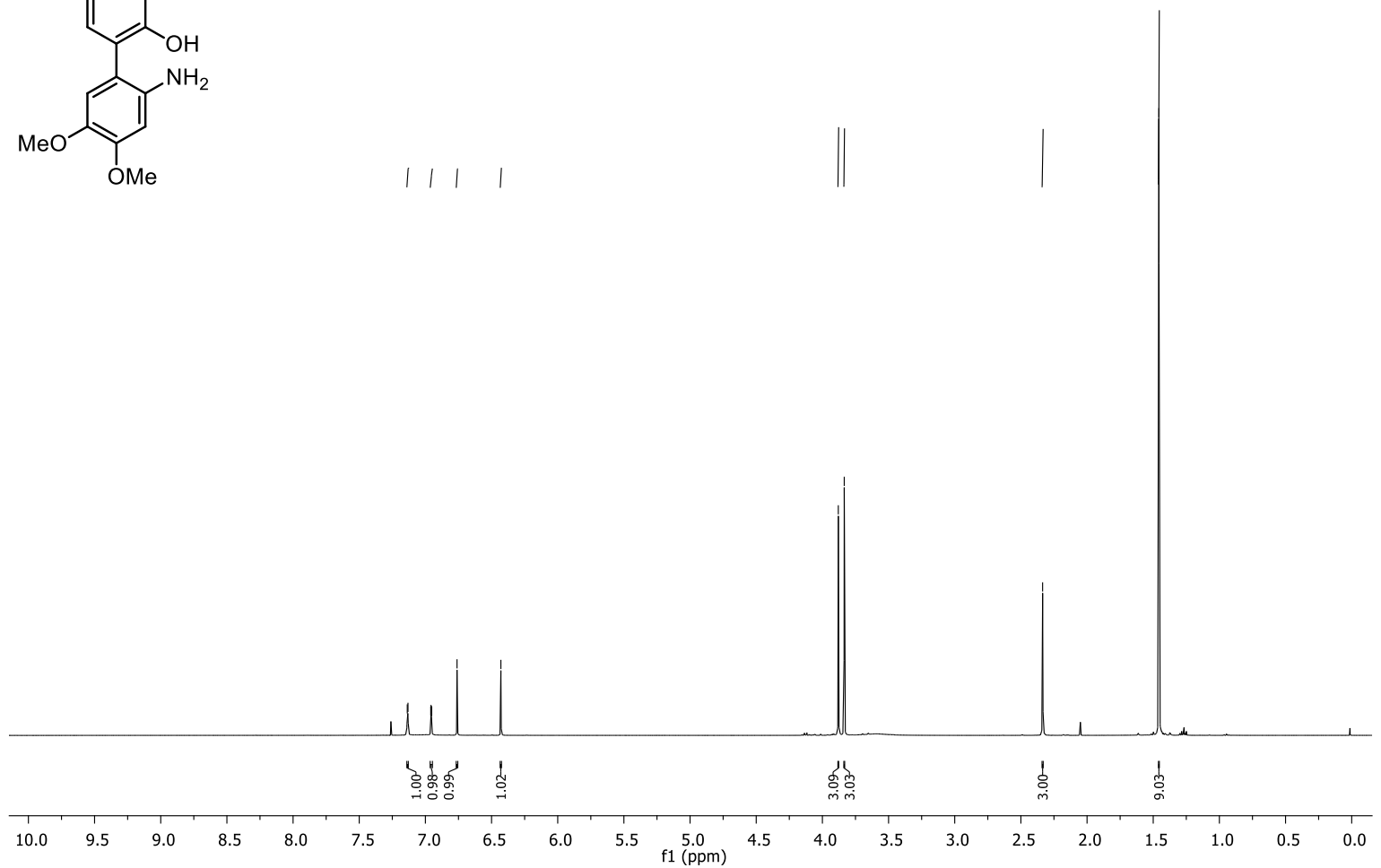

${ }^{13} \mathrm{C}$ - NMR spectrum of compound $26\left(100 \mathrm{MHz}, \mathrm{CDCl}_{3}\right)$

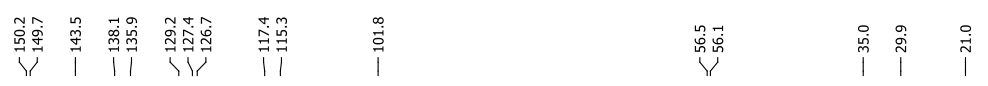
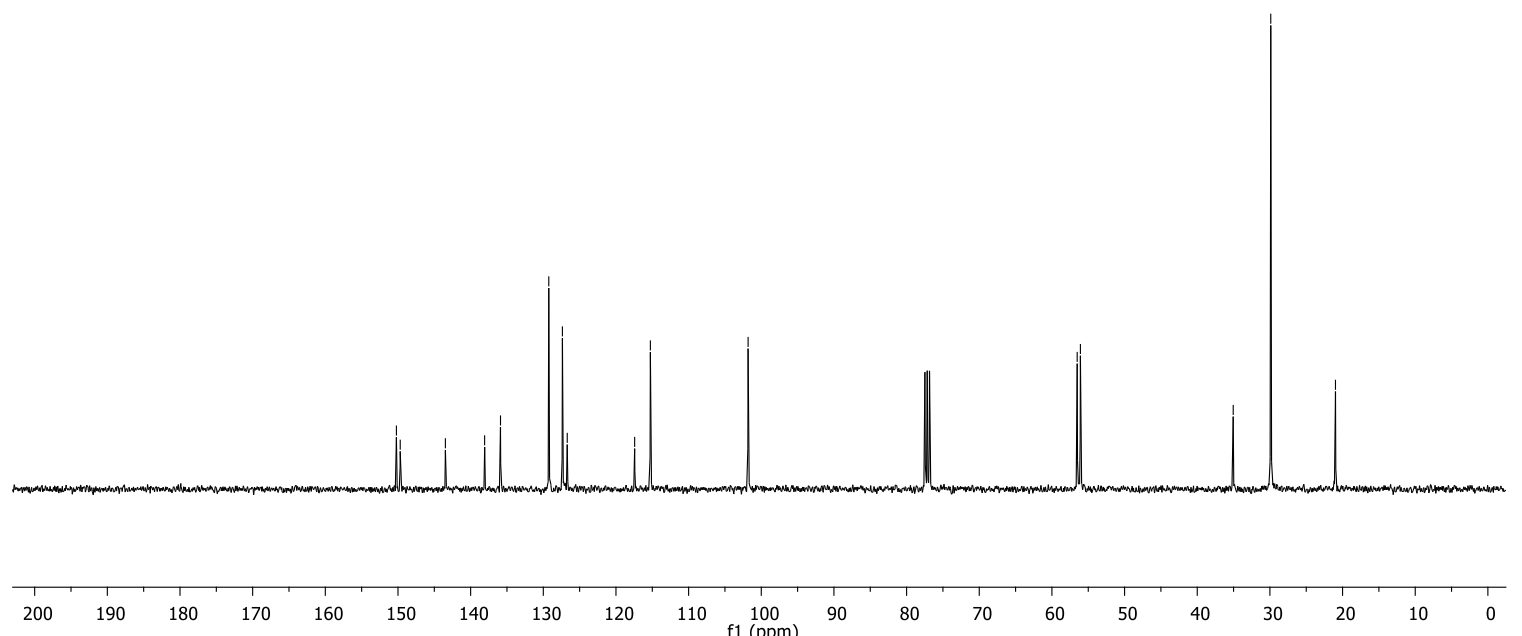
${ }^{1} \mathrm{H}$ - NMR spectrum of compound $27\left(400 \mathrm{MHz}, \mathrm{CDCl}_{3}\right)$
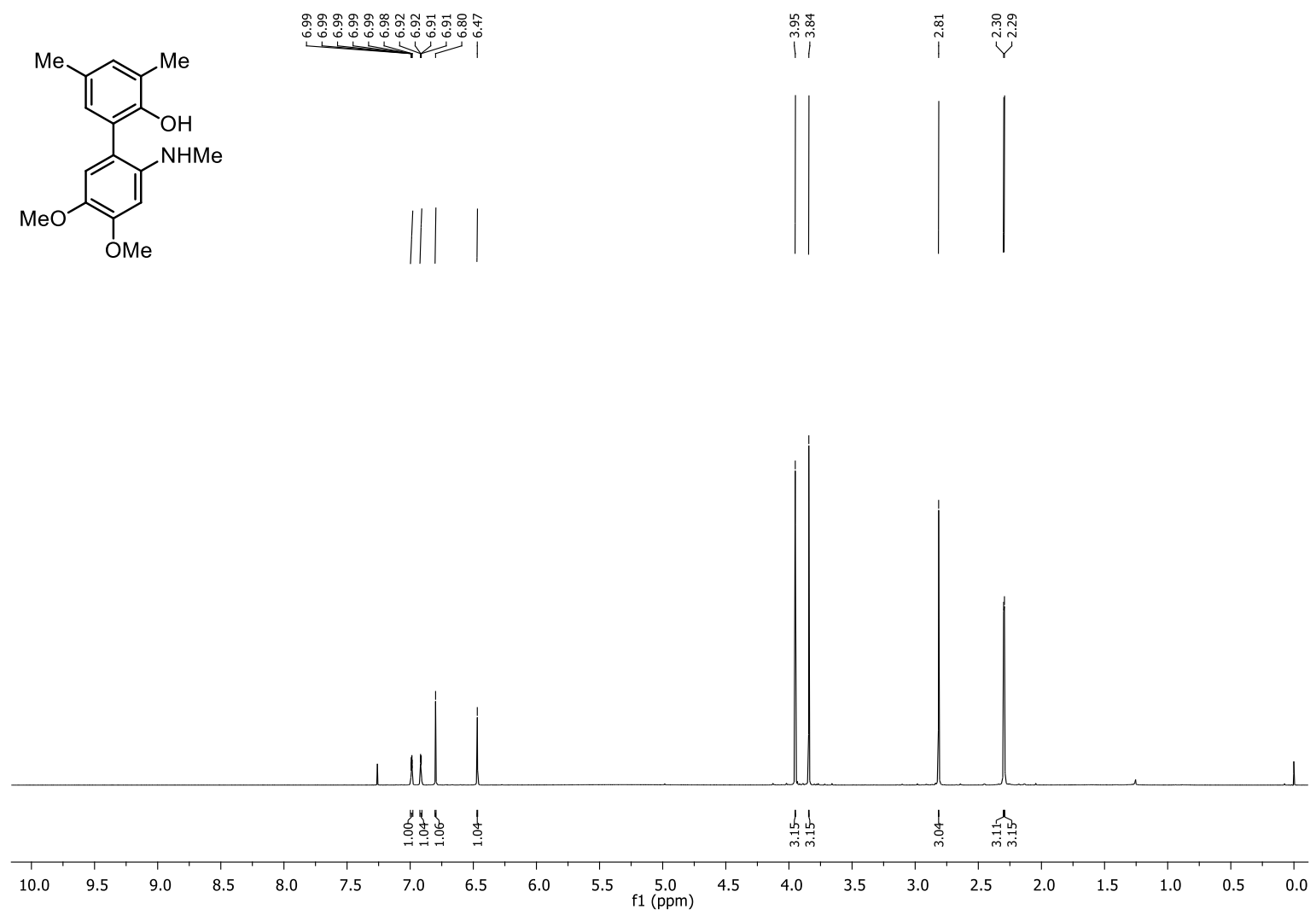

${ }^{13} \mathrm{C}$ - NMR spectrum of compound $27\left(100 \mathrm{MHz}, \mathrm{CDCl}_{3}\right)$

\begin{tabular}{|c|c|c|}
\hline 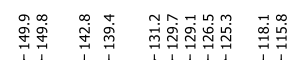 & $\stackrel{\widetilde{\alpha}}{\sigma}$ & 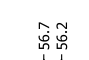 \\
\hline
\end{tabular}
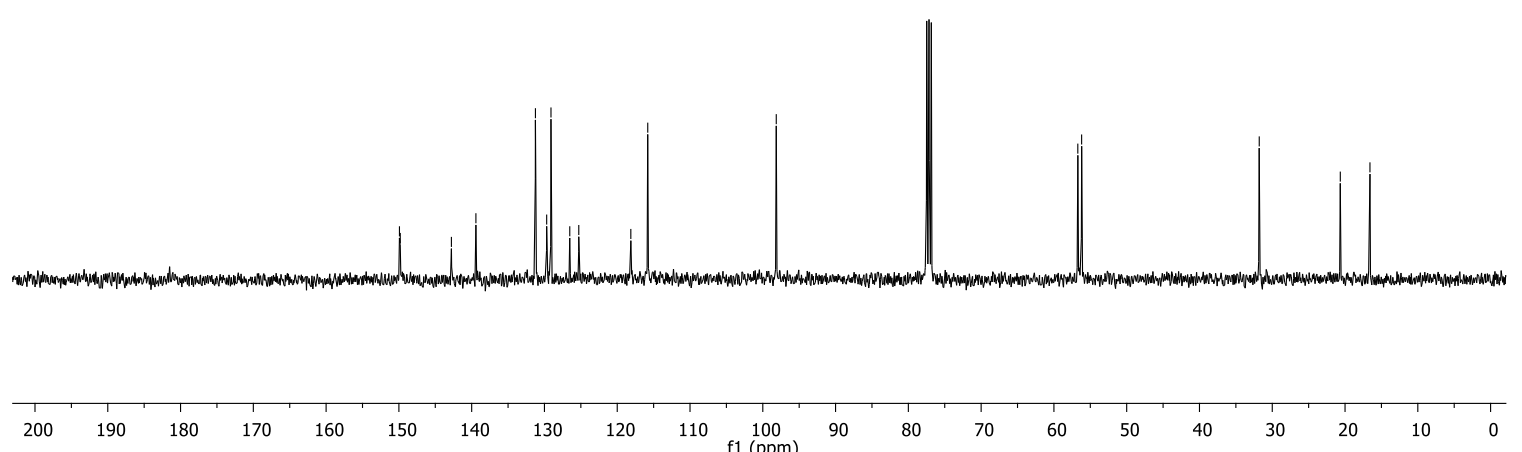
${ }^{1} \mathrm{H}$ - NMR spectrum of compound $28\left(400 \mathrm{MHz}, \mathrm{CDCl}_{3}\right)$
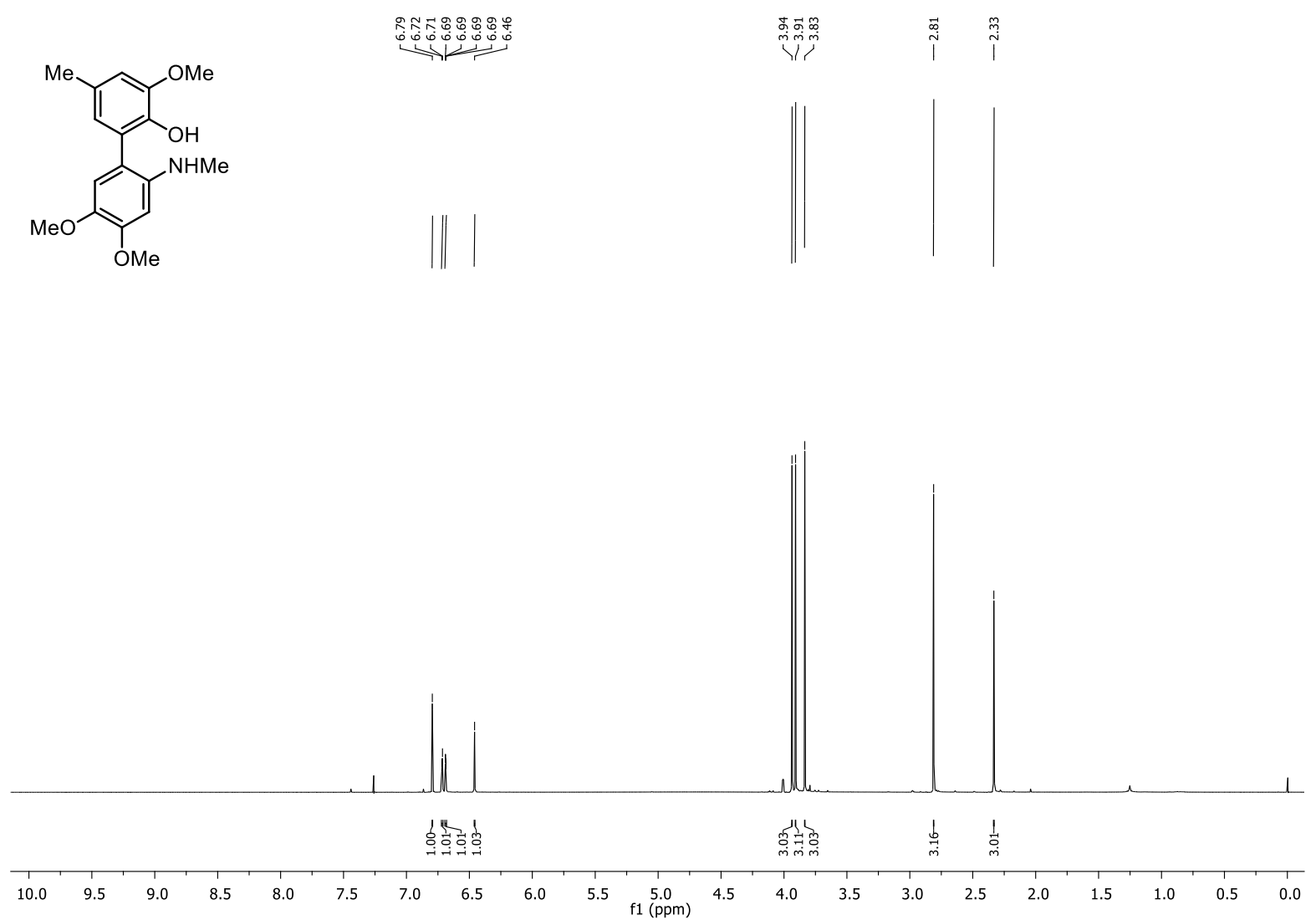

${ }^{13} \mathrm{C}$ - NMR spectrum of compound $28\left(100 \mathrm{MHz}, \mathrm{CDCl}_{3}\right)$

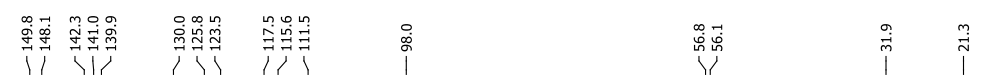

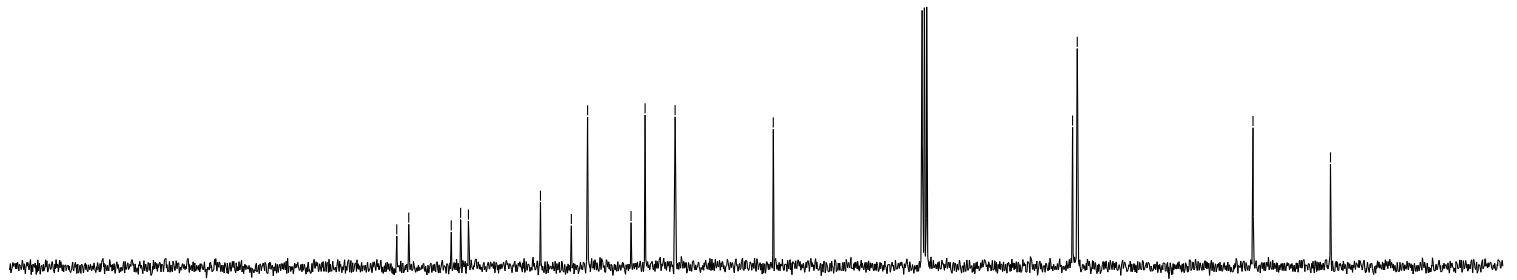


${ }^{1} \mathrm{H}$ - NMR spectrum of compound $29\left(400 \mathrm{MHz}, \mathrm{CDCl}_{3}\right)$<smiles>COc1cc(-c2cc([N+](=O)[O-])cc(OC)c2O)c(O)c(C(C)(C)C)c1</smiles>

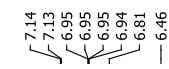

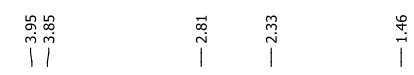
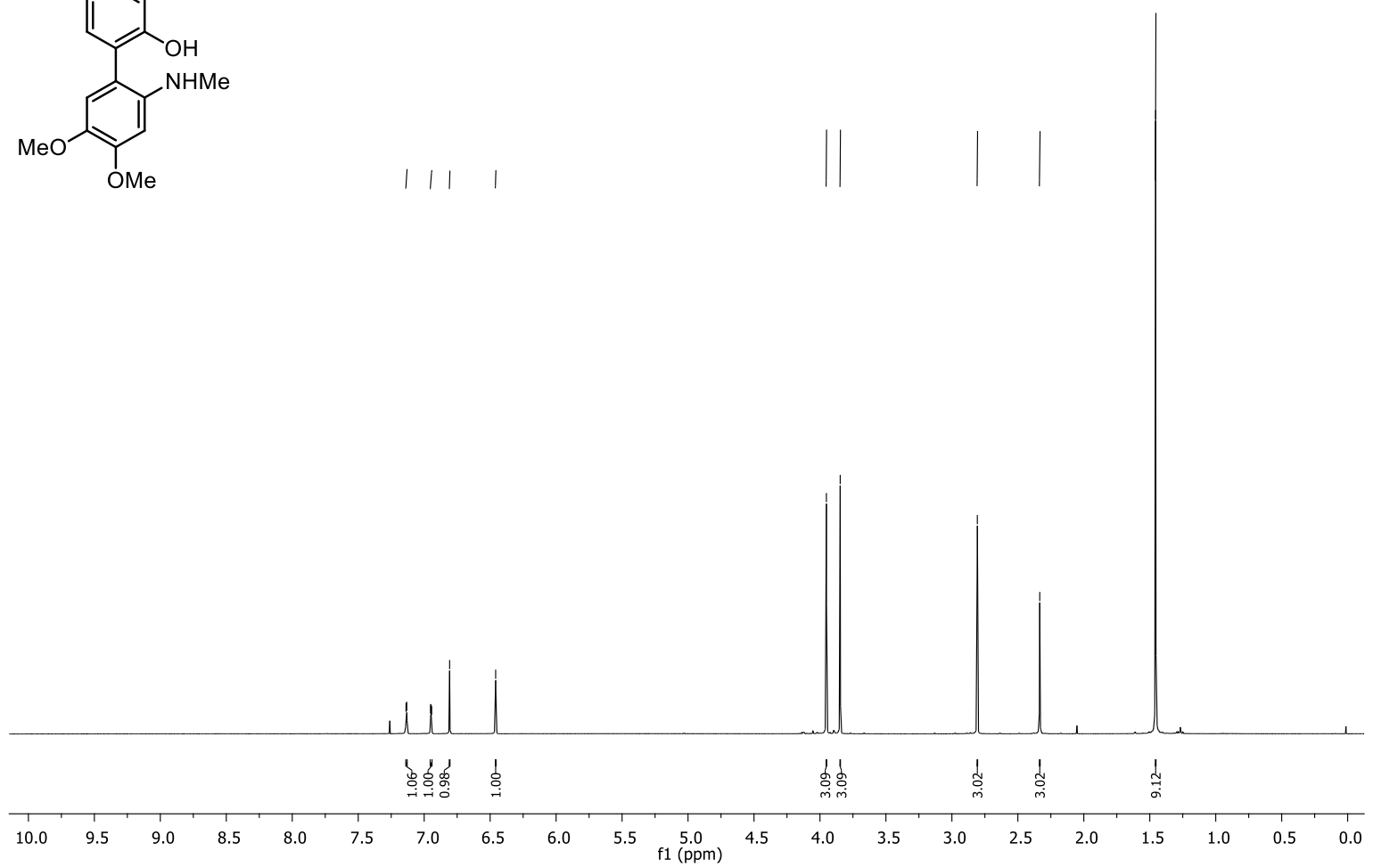

${ }^{13} \mathrm{C}$ - NMR spectrum of compound $29\left(100 \mathrm{MHz}, \mathrm{CDCl}_{3}\right)$

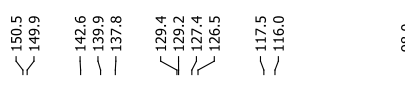

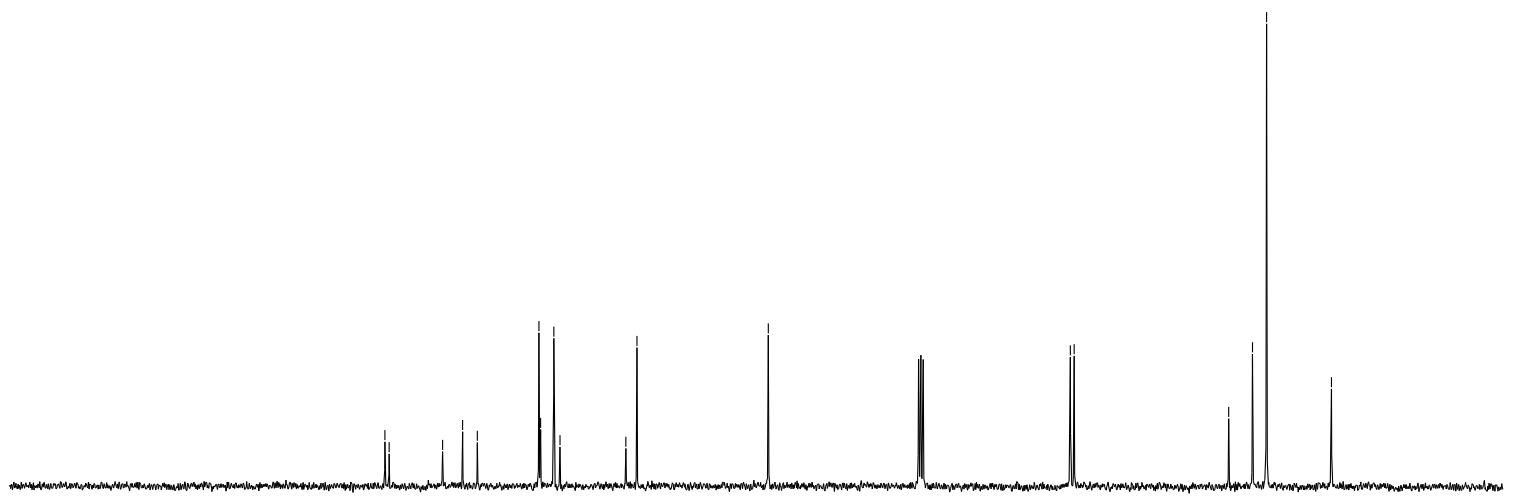


${ }^{1} \mathrm{H}$ - NMR spectrum of compound $\mathbf{3 0}\left(400 \mathrm{MHz}, \mathrm{CDCl}_{3}\right)$

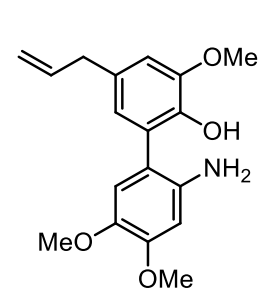

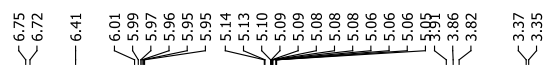

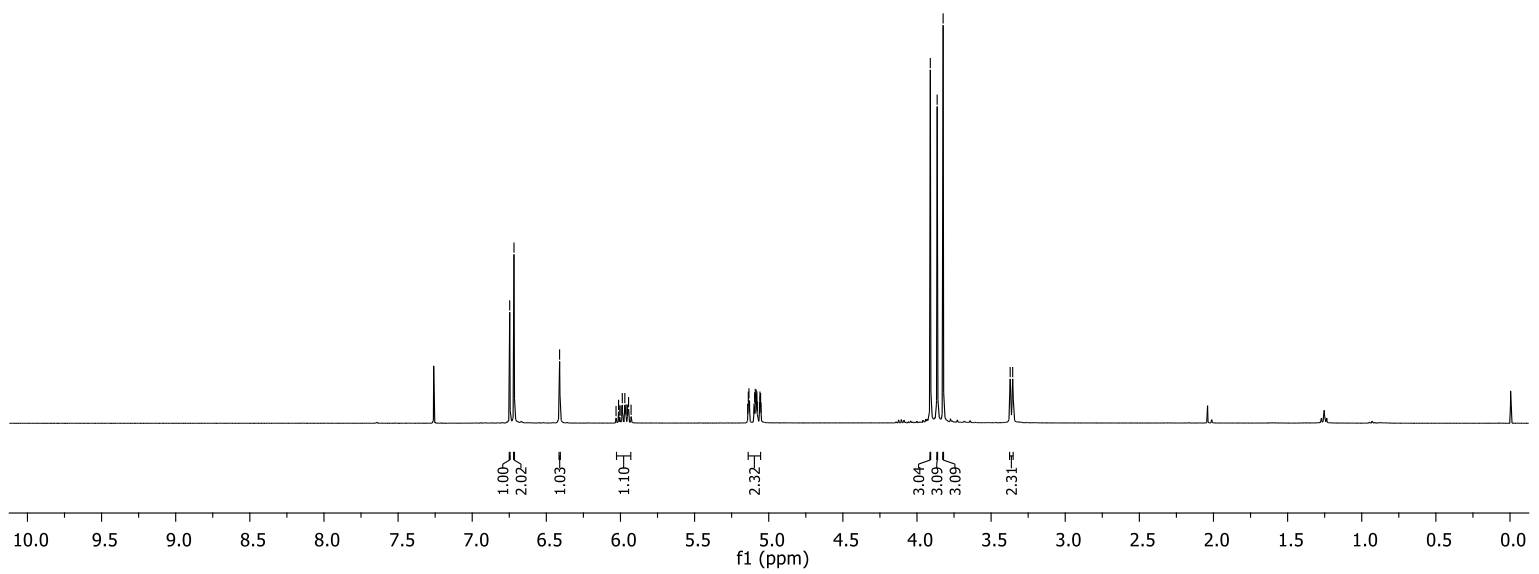

${ }^{13} \mathrm{C}$ - NMR spectrum of compound $30\left(100 \mathrm{MHz}, \mathrm{CDCl}_{3}\right)$

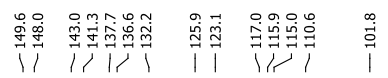

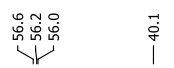
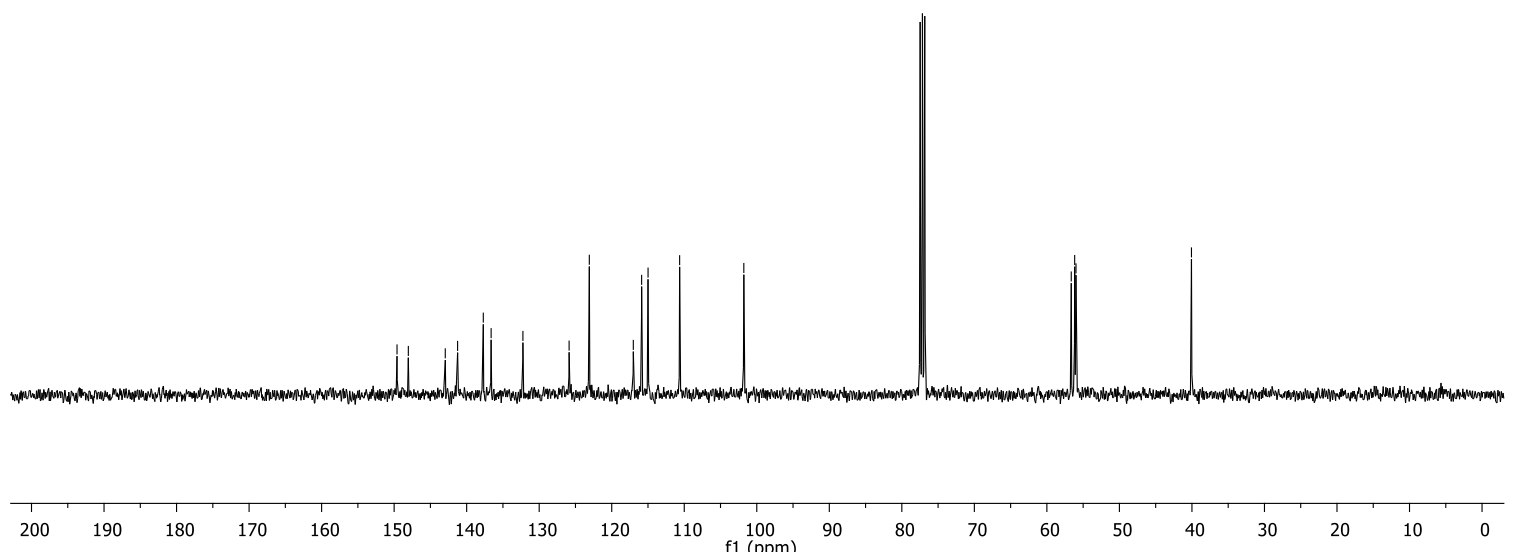
${ }^{1} \mathrm{H}$ - NMR spectrum of compound $31\left(400 \mathrm{MHz}, \mathrm{CDCl}_{3}\right)$

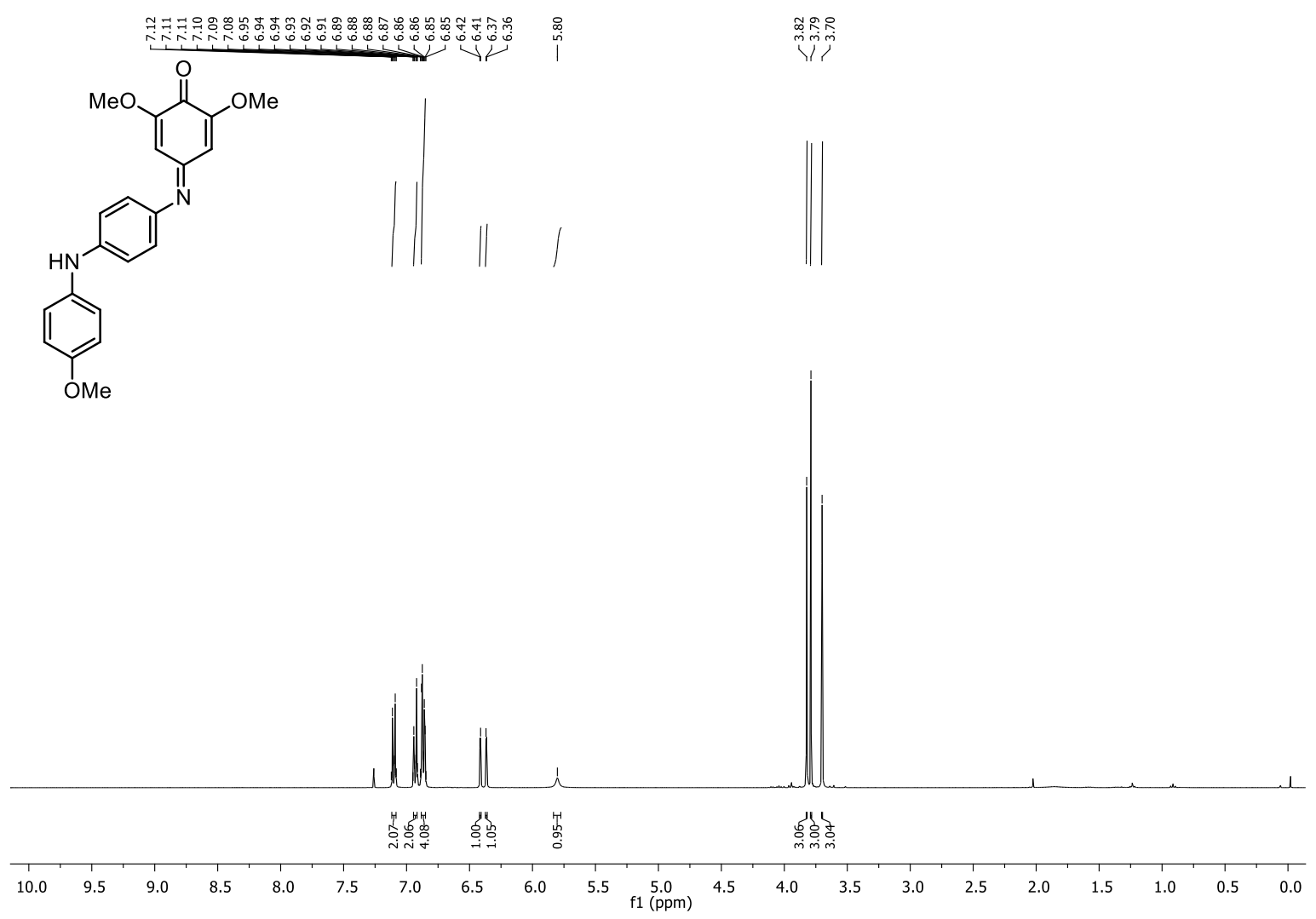

${ }^{13} \mathrm{C}$ - NMR spectrum of compound $31\left(100 \mathrm{MHz}, \mathrm{CDCl}_{3}\right)$

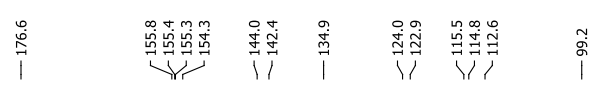

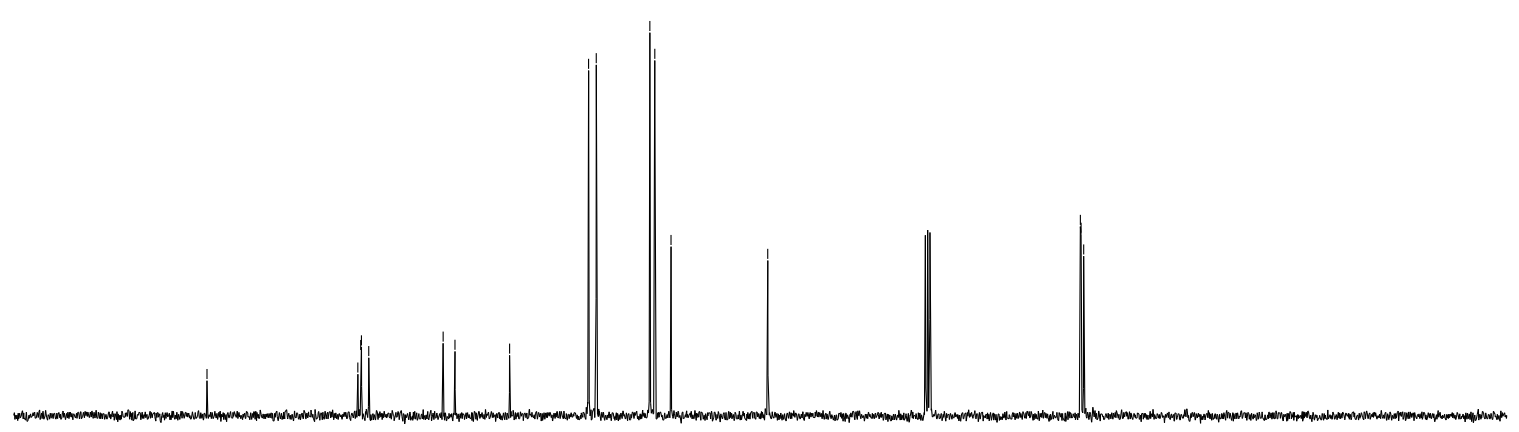

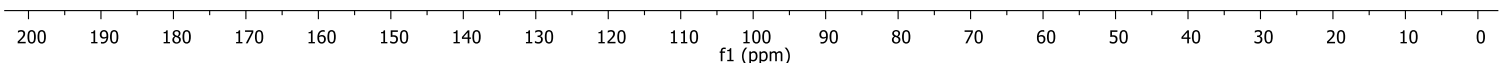


${ }^{1} \mathrm{H}$ - NMR spectrum of compound $32\left(400 \mathrm{MHz}, \mathrm{CDCl}_{3}\right)$

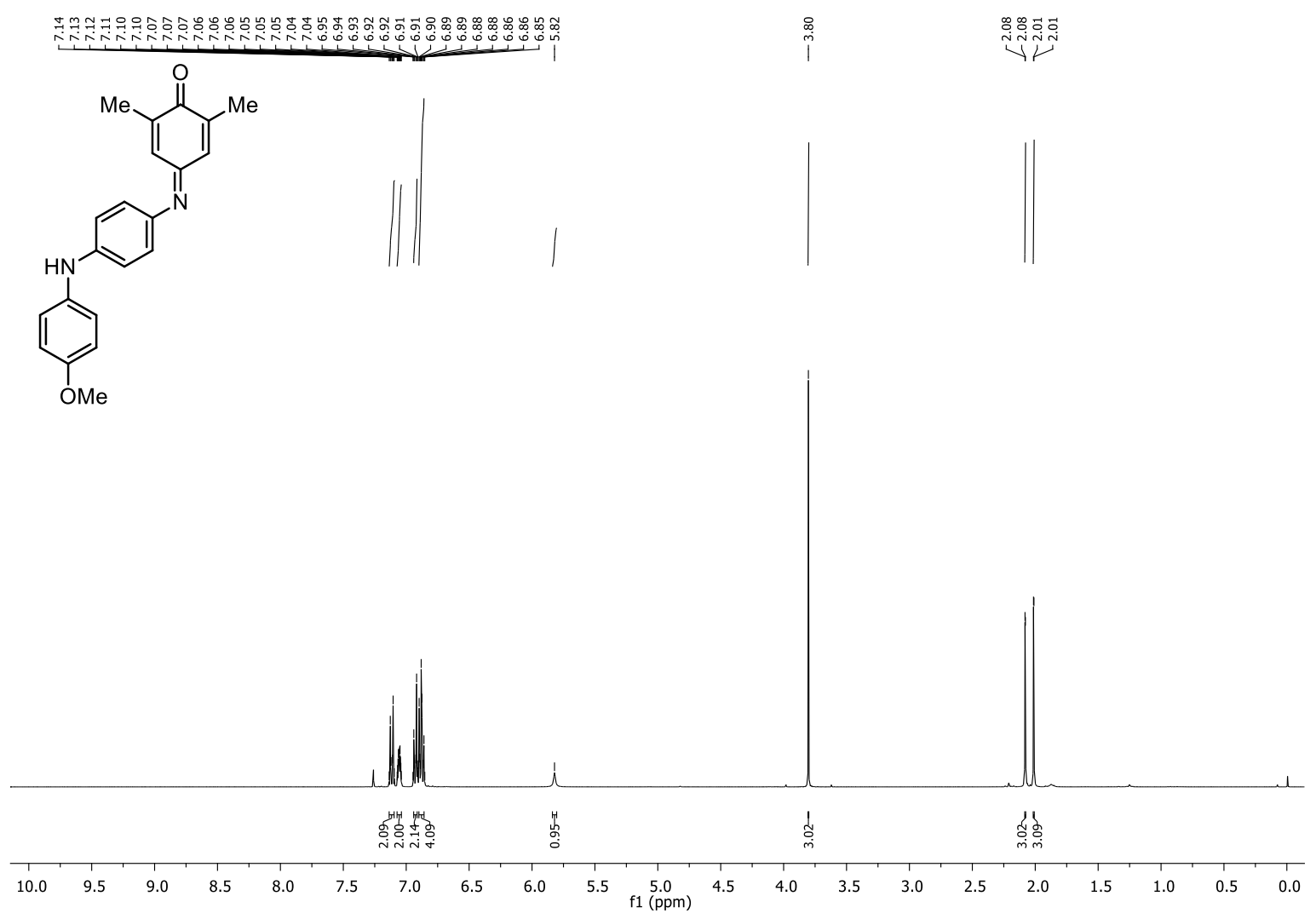

${ }^{13} \mathrm{C}$ - NMR spectrum of compound $32\left(100 \mathrm{MHz}, \mathrm{CDCl}_{3}\right)$
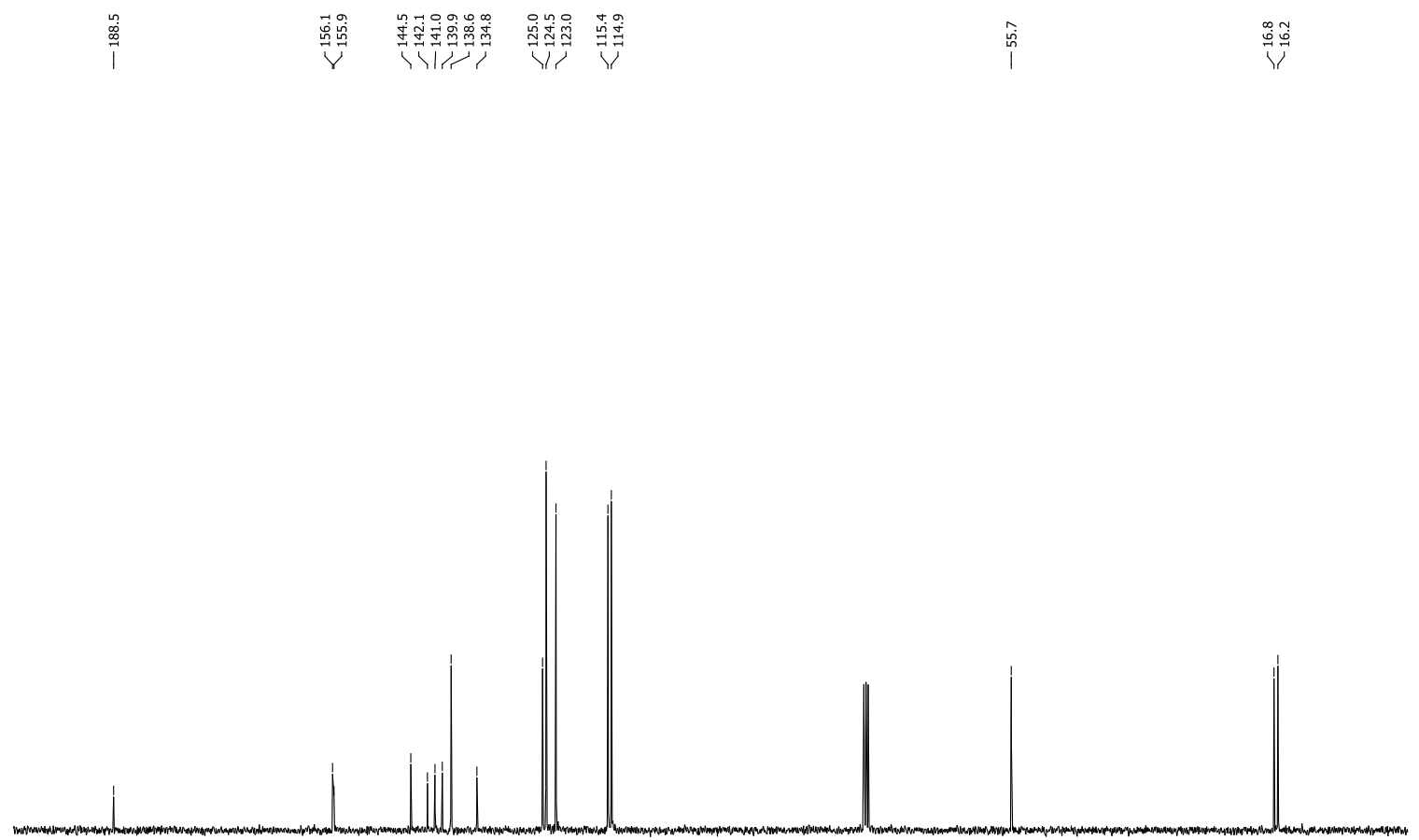

200

$\begin{array}{llllllllll}90 & 180 & 170 & 160 & 150 & 140 & 130 & 120 & 110 & \begin{array}{l}100 \\ \mathrm{f} 1(\mathrm{ppm})\end{array}\end{array}$ 
${ }^{1} \mathrm{H}$ - NMR spectrum of compound $33\left(400 \mathrm{MHz}, \mathrm{CDCl}_{3}\right)$

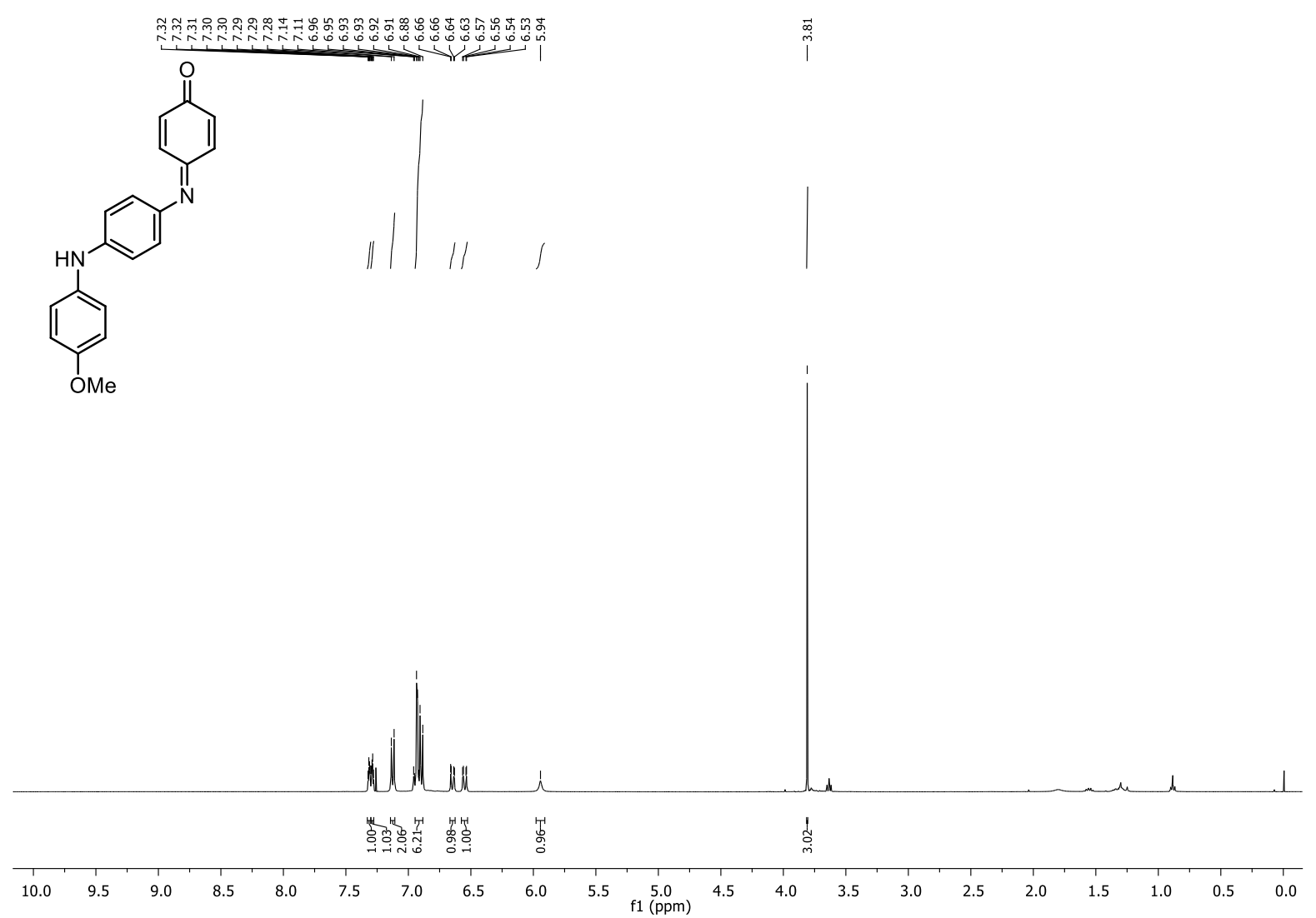

${ }^{13} \mathrm{C}$ - NMR spectrum of compound $33\left(100 \mathrm{MHz}, \mathrm{CDCl}_{3}\right)$

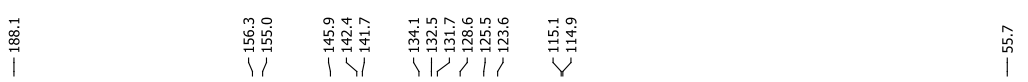

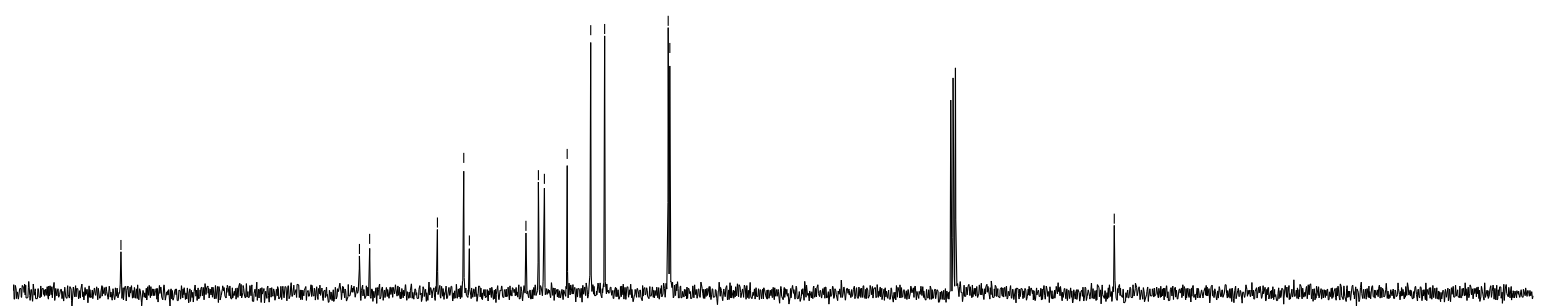

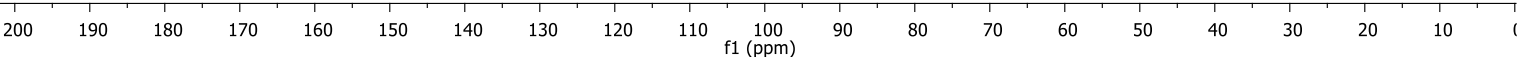


${ }^{1} \mathrm{H}-\mathrm{NMR}$ spectrum of two isomeric conformations of compound $34\left(400 \mathrm{MHz}, \mathrm{CDCl}_{3}\right)$
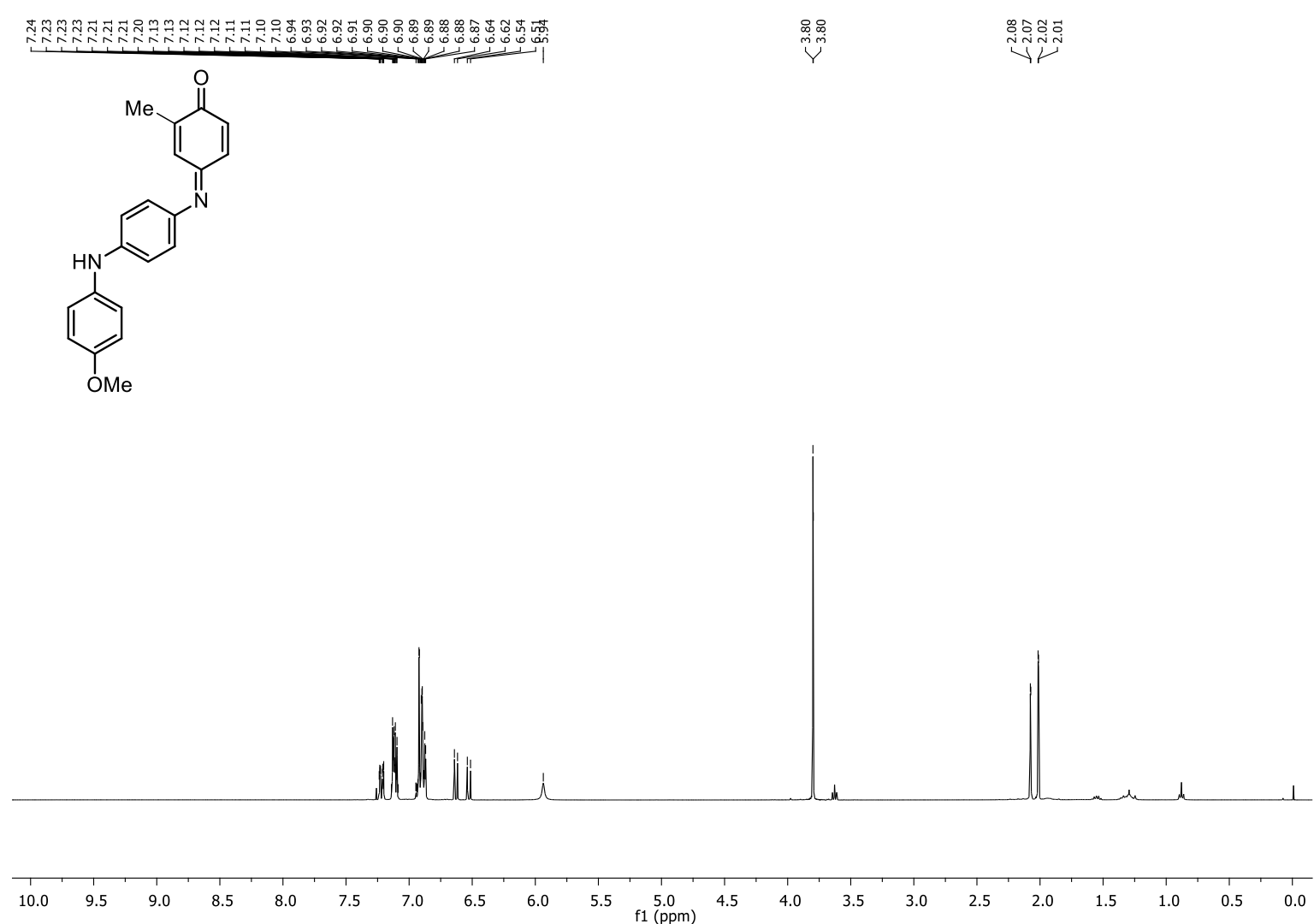

${ }^{13} \mathrm{C}$ - NMR spectrum of two isomeric conformations of compound $34\left(100 \mathrm{MHz}, \mathrm{CDCl}_{3}\right)$ $\stackrel{\substack{m \\ \infty}}{\substack{\infty \\ \hdashline}}$

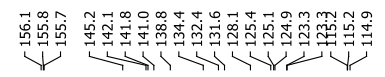

$\stackrel{\substack{0.0 \\ \text { i }}}{1}$

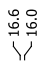

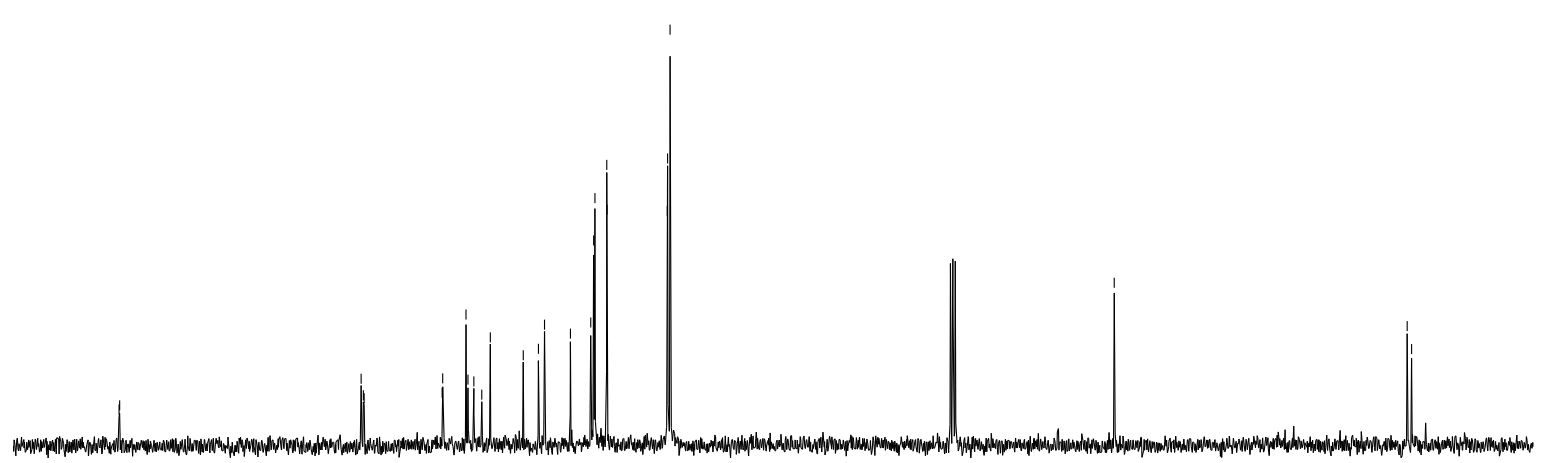


${ }^{1} \mathrm{H}$ - NMR spectrum of compound $35\left(400 \mathrm{MHz}, \mathrm{CDCl}_{3}\right)$
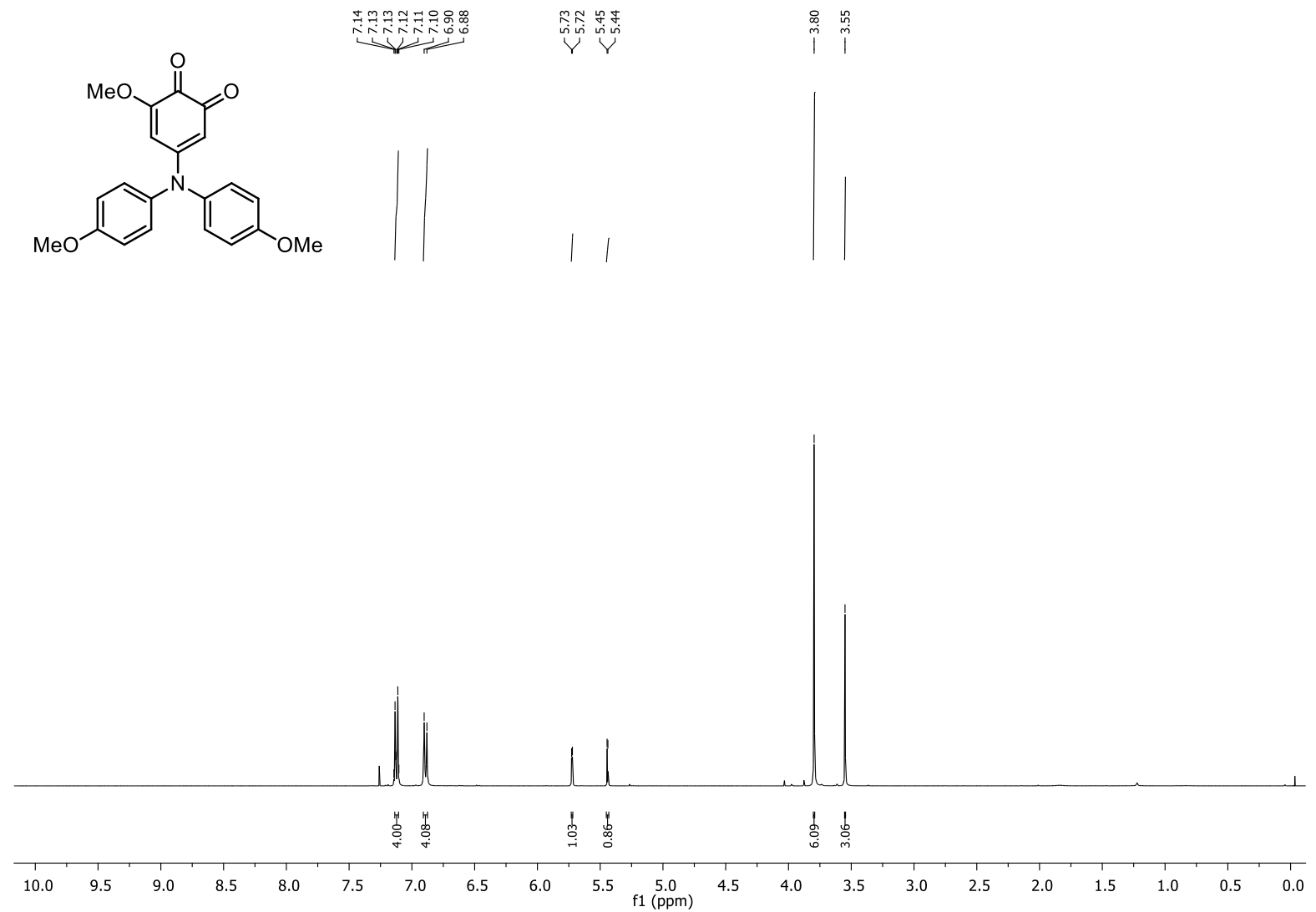

${ }^{13} \mathrm{C}$ - NMR spectrum of compound $35\left(100 \mathrm{MHz}, \mathrm{CDCl}_{3}\right)$

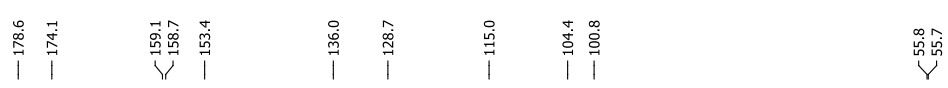

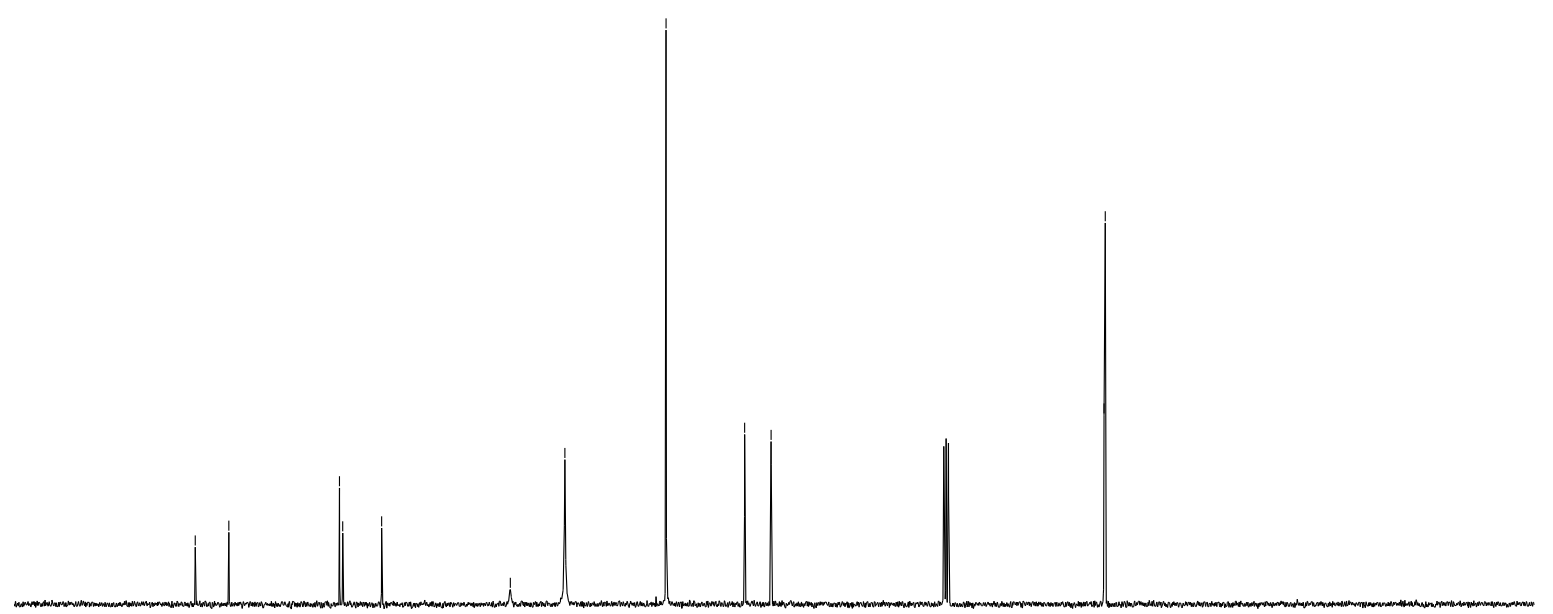


${ }^{1} \mathrm{H}$ - NMR spectrum of compound $36\left(400 \mathrm{MHz}, \mathrm{CDCl}_{3}\right)$<smiles>COc1ccc(N(c2ccc(OC)cc2)c2cc(C=O)c(OC)c([N+](=O)[O-])c2)cc1</smiles>

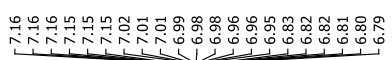
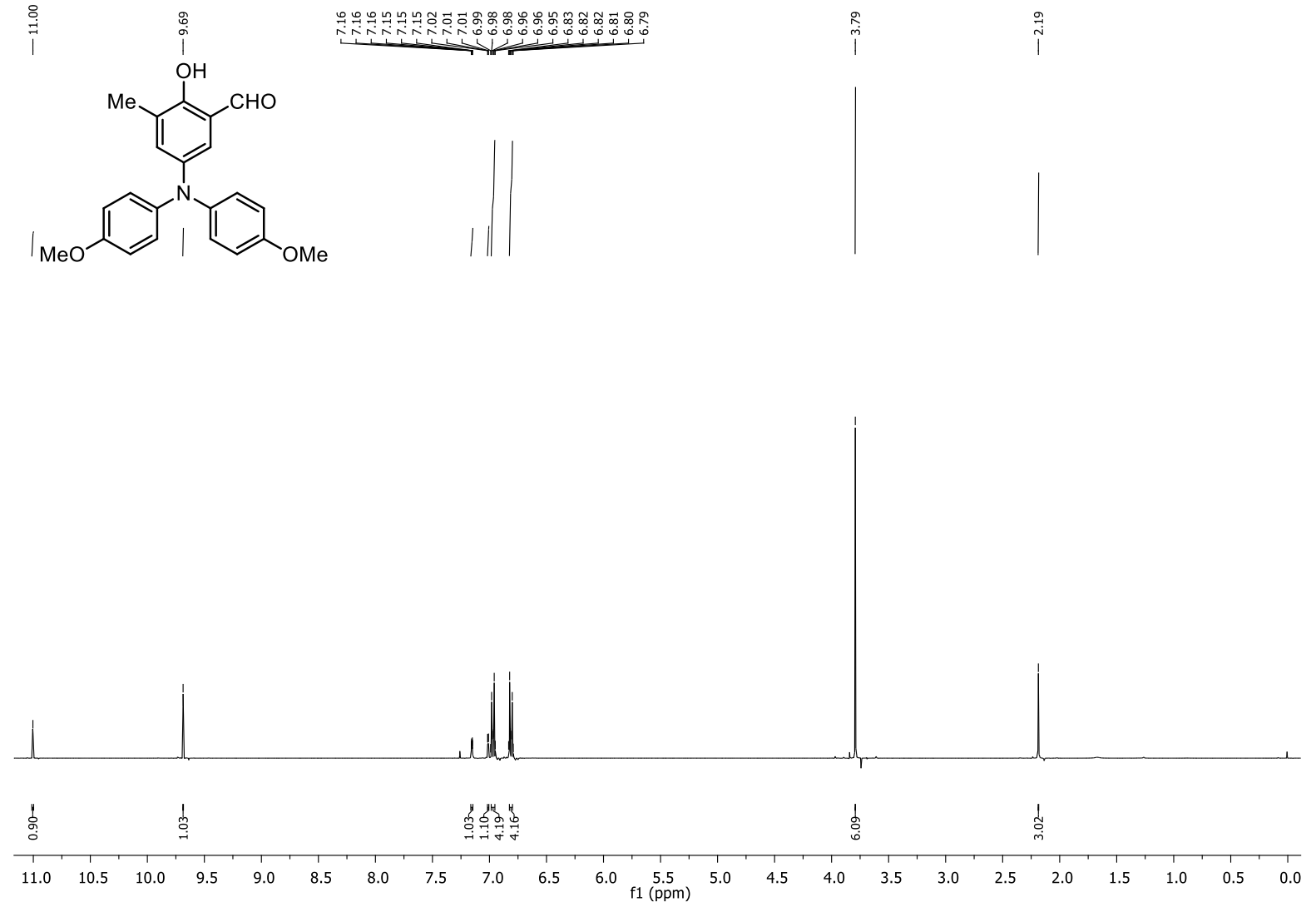

${ }^{13} \mathrm{C}$ - NMR spectrum of compound $36\left(100 \mathrm{MHz}, \mathrm{CDCl}_{3}\right)$

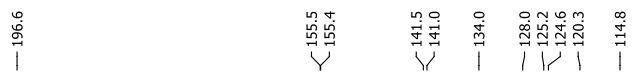
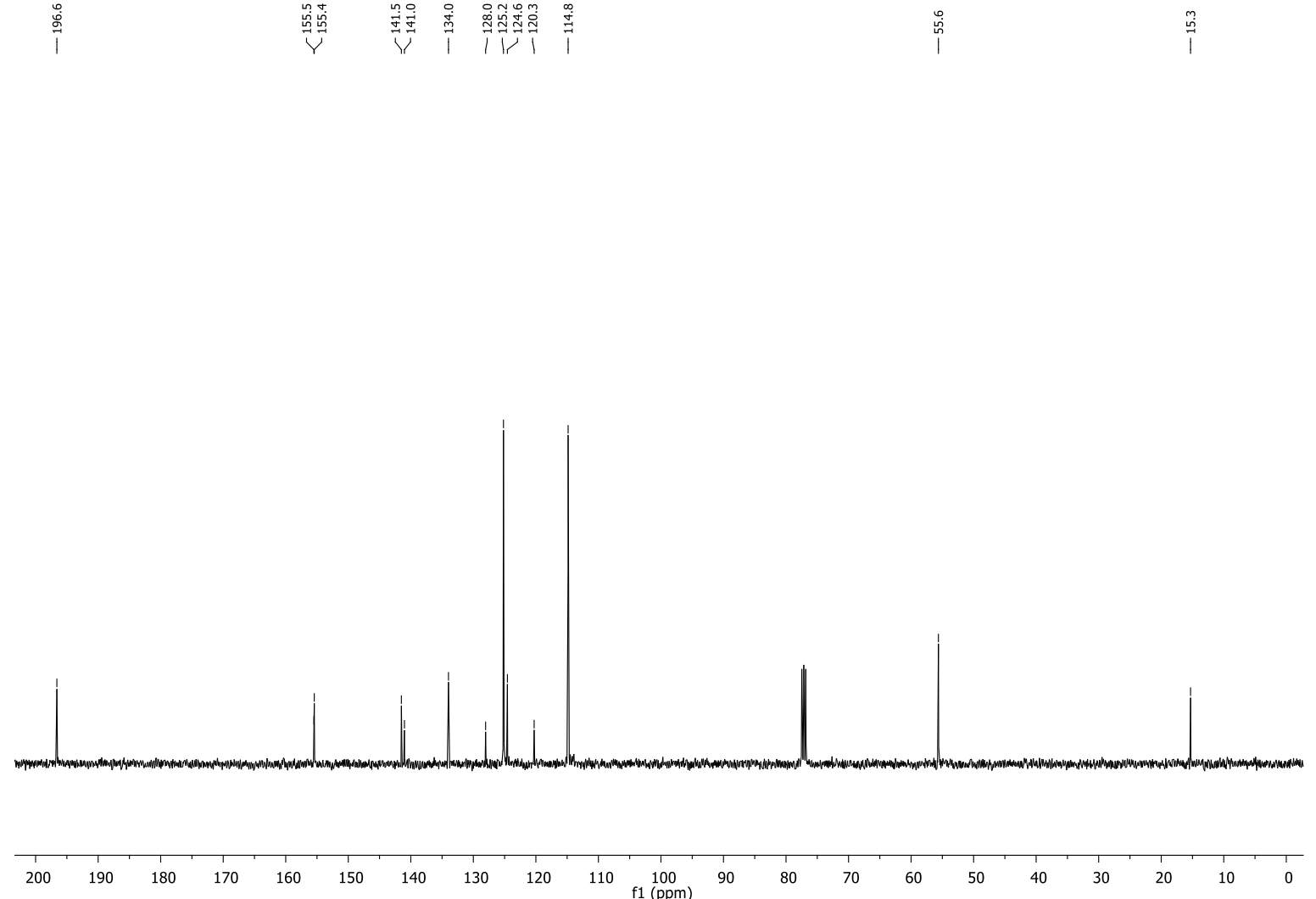
${ }^{1} \mathrm{H}$ - NMR spectrum of compound 13a $\left(400 \mathrm{MHz}, \mathrm{CDCl}_{3}\right)$
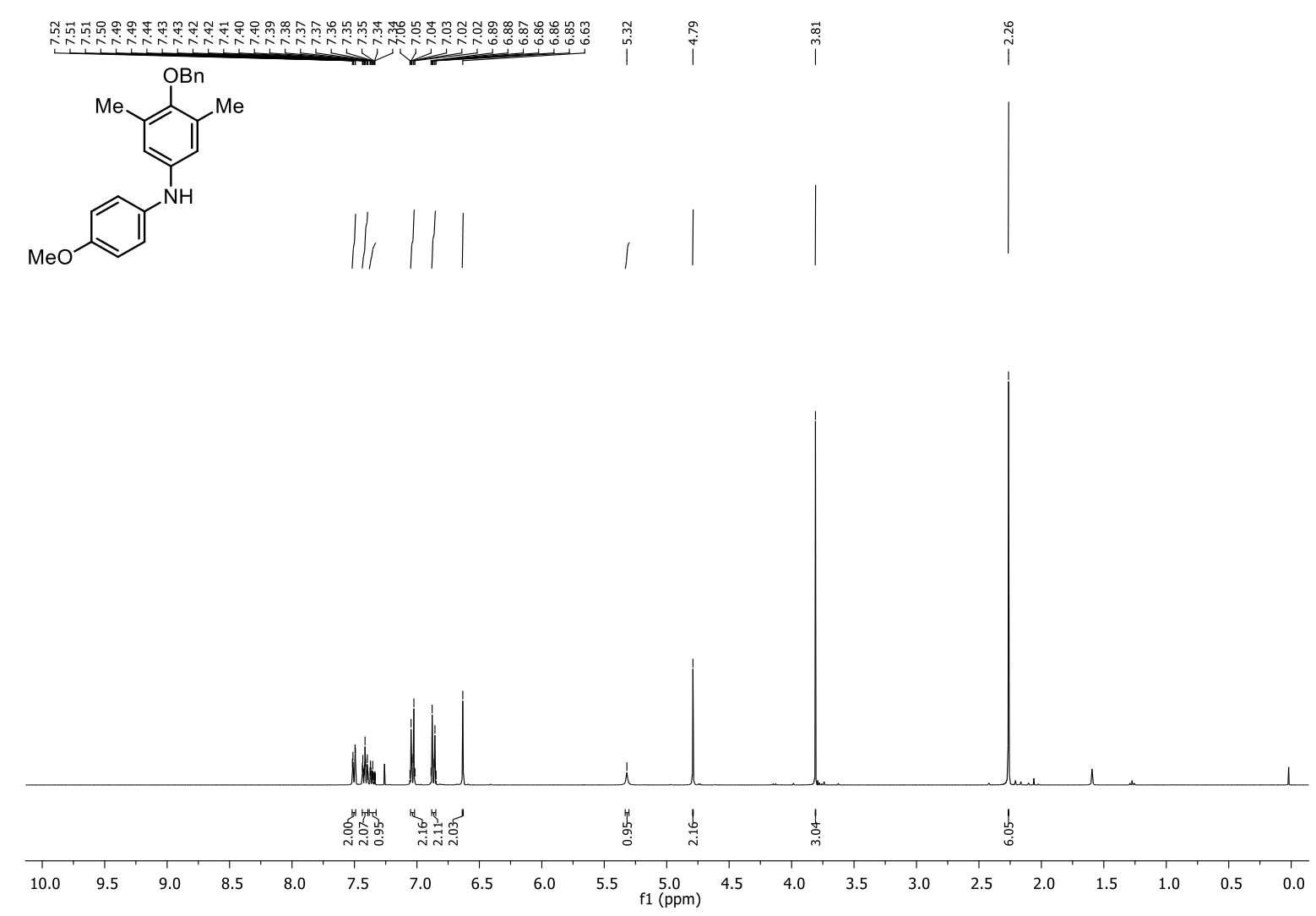

${ }^{13} \mathrm{C}$ - NMR spectrum of compound $\mathbf{1 3 a}\left(100 \mathrm{MHz}, \mathrm{CDCl}_{3}\right)$

|
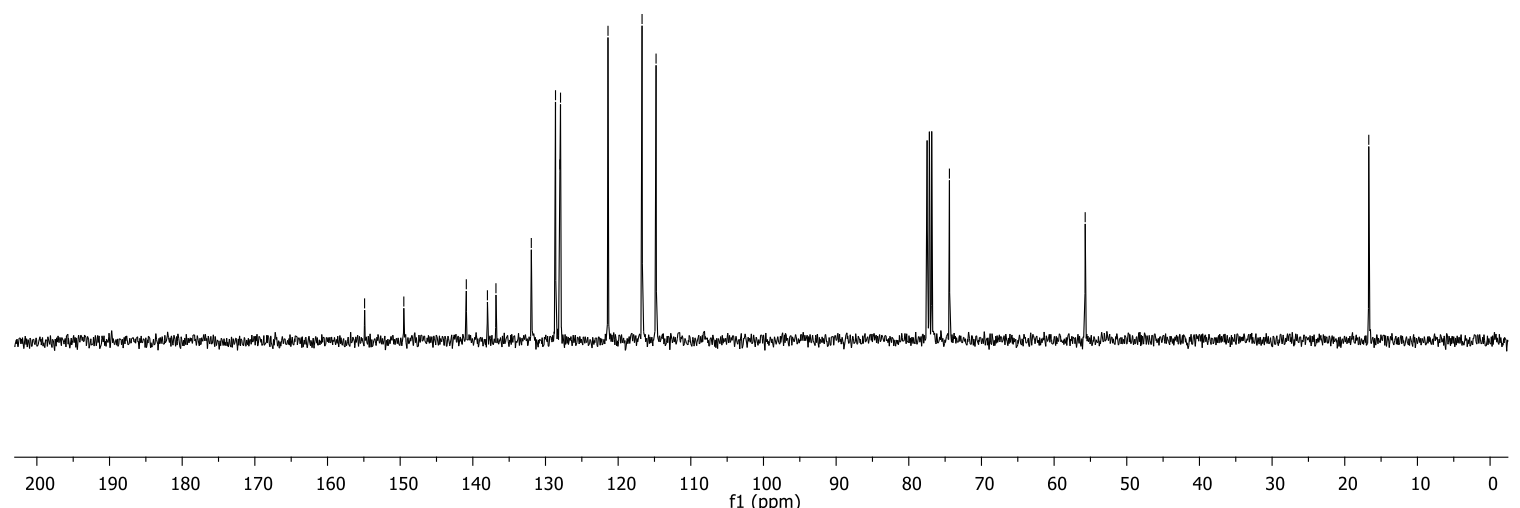
${ }^{1} \mathrm{H}$ - NMR spectrum of compound $37\left(400 \mathrm{MHz}, \mathrm{CDCl}_{3}\right)$<smiles>CCOC(=O)Oc1c(OC)cc(Nc2ccc(OC)cc2)cc1OC</smiles>

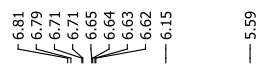

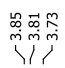

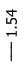

OMe

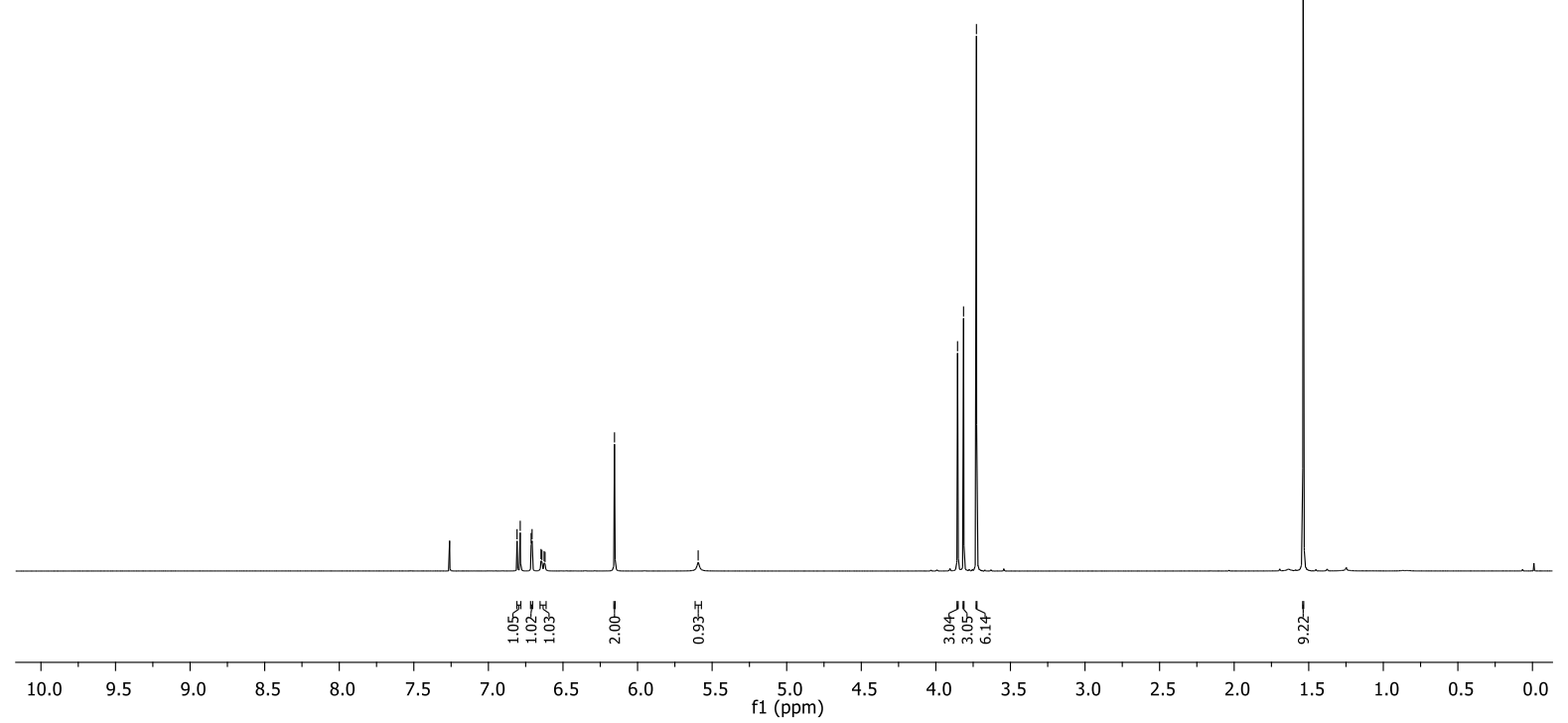

${ }^{13} \mathrm{C}$ - NMR spectrum of compound $37\left(100 \mathrm{MHz}, \mathrm{CDCl}_{3}\right)$

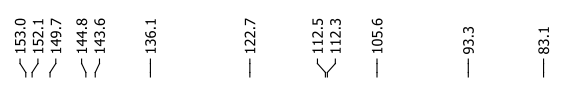

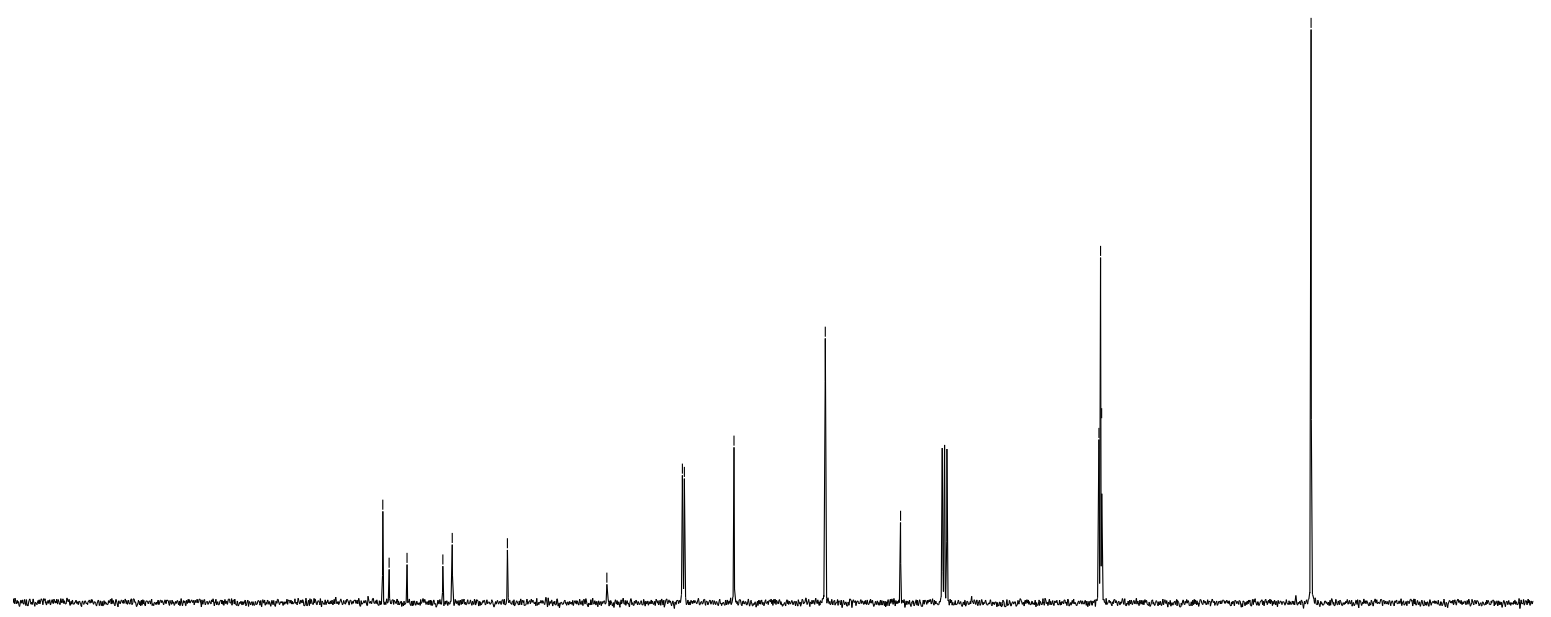

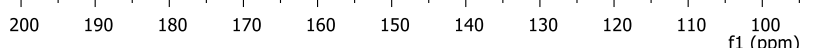


${ }^{1} \mathrm{H}$ - NMR spectrum of compound $38\left(400 \mathrm{MHz}, \mathrm{CDCl}_{3}\right)$<smiles>COc1ccc(Nc2cc(OC)c(OC(C)=O)c(OC)c2)cc1</smiles>

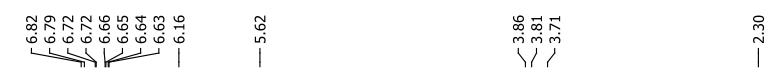

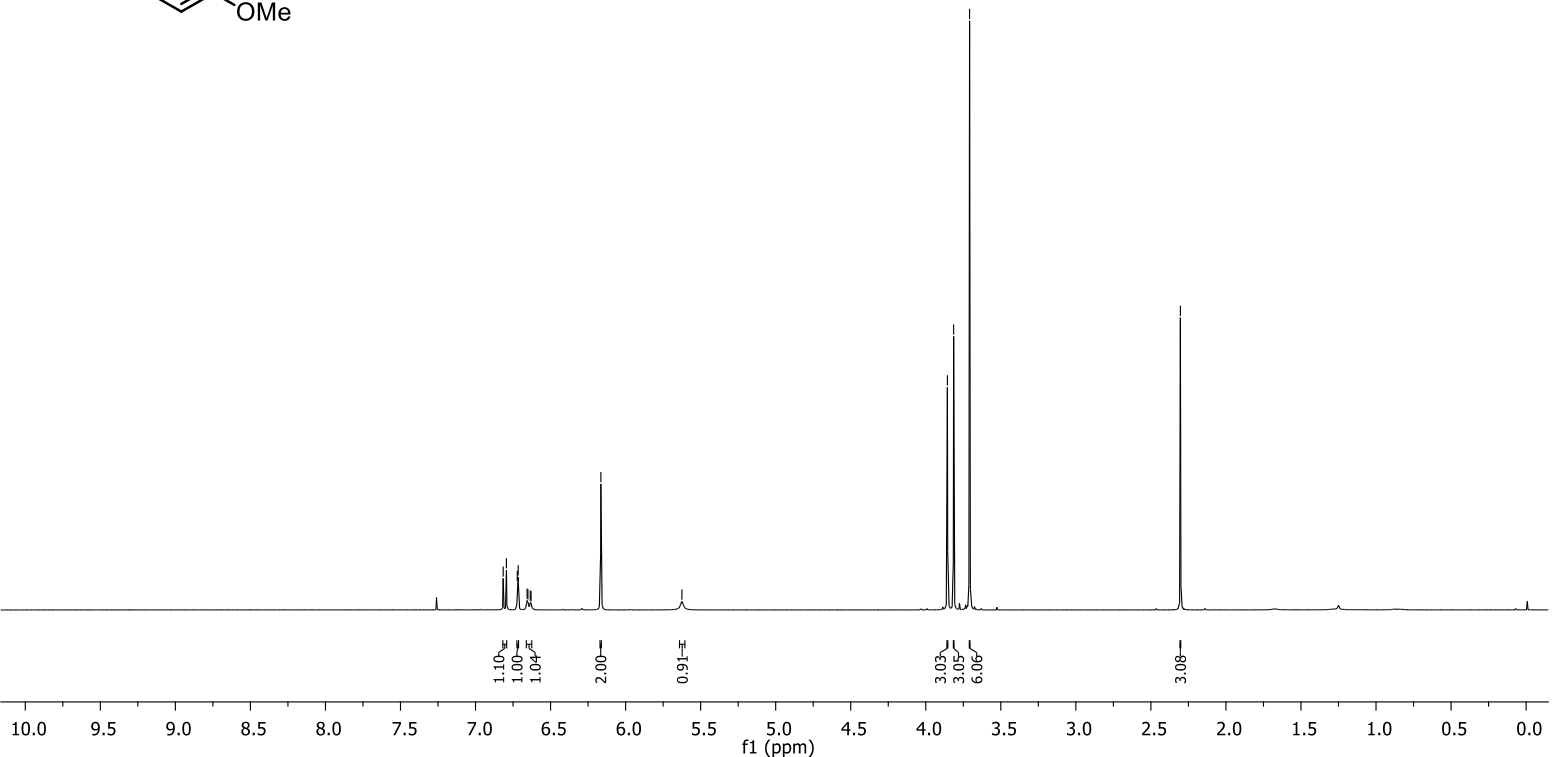

${ }^{13} \mathrm{C}$ - NMR spectrum of compound $38\left(100 \mathrm{MHz}, \mathrm{CDCl}_{3}\right)$

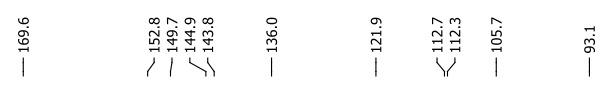

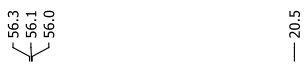
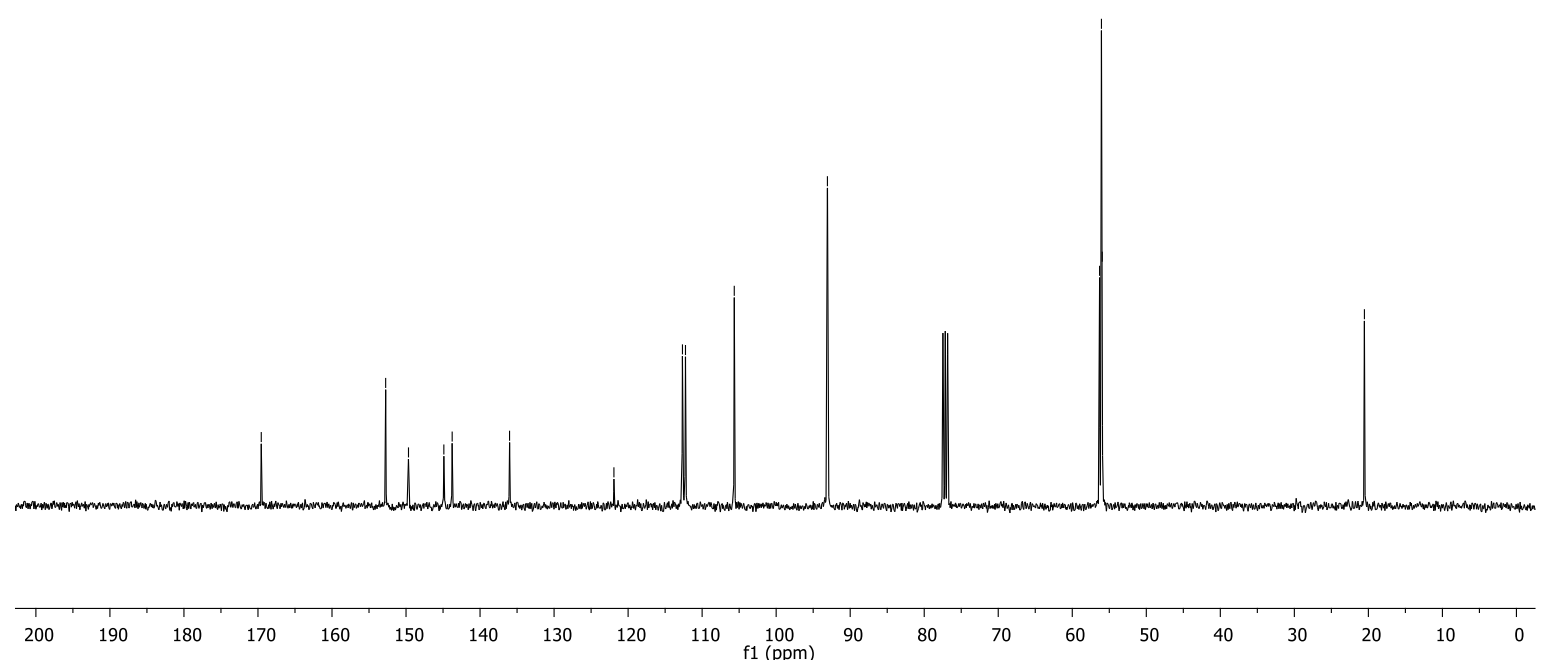